\title{
Redefining replication in multi-ancestry genome-wide association studies
}

Samuel Pattillo Smith ${ }^{1,2}$, Sahar Shahamatdar ${ }^{1,2}$, Wei Cheng ${ }^{1,2}$, Selena Zhang ${ }^{1}$, Joseph Paik $^{1}$, Misa Graff ${ }^{3}$, Christopher Haiman ${ }^{4}$, T.C. Matise ${ }^{5}$, Kari E North ${ }^{3}$, Ulrike Peters ${ }^{6}$, Eimear Kenny ${ }^{7,8,9,10}$, Chris Gignoux ${ }^{11}$, Genevieve Wojcik ${ }^{12}$, Lorin Crawford ${ }^{1,13, *}$, and Sohini Ramachandran ${ }^{1,2, *, \dagger}$

${ }^{1}$ Center for Computational Molecular Biology, Brown University, Providence RI

${ }^{2}$ Department of Ecology and Evolutionary Biology, Brown University, Providence RI ${ }^{3}$ Department of Epidemiology, University of North Carolina - Chapel Hill, Chapel Hill NC ${ }^{4}$ Department of Preventative Medicine, University of Southern California, Los Angeles CA

${ }^{5}$ Department of Genetics, Rutgers University, Piscataway NJ

${ }^{6}$ Public Health Sciences Division, Fred Hutchinson Cancer Research Center, Seattle WA ${ }^{7}$ The Center for Genomic Health, Icahn School of Medicine at Mount Sinai, New York City NY

${ }^{8}$ The Charles Bronfman Institute for Personalized Medicine, Icahn School of Medicine at Mount Sinai, New York City NY

${ }^{9}$ Department of Medicine, Icahn School of Medicine at Mount Sinai, New York City NY ${ }^{10}$ Department of Genetics and Genomic Sciences, Icahn School of Medicine at Mount Sinai, New York City NY

${ }^{11}$ Division of Biomedical Informatics and Personalized Medicine, University of Colorado, Denver CO

${ }^{12}$ Department of Epidemiology, Johns Hopkins University, Baltimore MD ${ }^{13}$ Microsoft Research New England, Cambridge MA *indicates these authors contributed equally

${ }^{\dagger}$ To whom correspondence should be addressed: sramachandran@brown.edu

Last edited April 20, 2021

\begin{abstract}
Since 2005, genome-wide association (GWA) datasets have been largely biased toward sampling European ancestry individuals, and recent studies have shown that GWA results estimated from European ancestry individuals apply heterogeneously in non-European ancestry individuals. Here, we argue that enrichment analyses which aggregate SNP-level association statistics at multiple genomic scales- to genes and pathways - have been overlooked and can generate biologically interpretable hypotheses regarding the genetic basis of complex trait architecture. We illustrate examples of the insights generated by enrichment analyses while studying 25 continuous traits assayed in 566,786 individuals from seven self-identified human ancestries in the UK Biobank and the Biobank Japan, as well as 44,348 admixed individuals from
\end{abstract}


the PAGE consortium including cohorts of African-American, Hispanic and Latin American, Native Hawaiian, and American Indian/Alaska Native individuals. By testing for statistical associations at multiple genomic scales, enrichment analyses also illustrate the importance of reconciling contrasting results from association tests, heritability estimation, and prediction models in order to make personalized medicine a reality for all.

\section{Introduction}

Over the past two decades, funding agencies and biobanks around the world have made enormous investments to generate large-scale datasets of genotypes, exomes, and whole-genome sequences from diverse human ancestries that are merged with medical records and quantitative trait measurements 1 . 8 . However, analyses of such datasets are usually limited to applications of the standard genome-wide association (GWA) SNP-level association analyses, in which SNPs are tested one-by-one for significant association with a phenotype 11 (Table 1). Yet, even in the largest available multi-ancestry biobanks, GWA analyses fail to offer a comprehensive view of genetic trait architecture among human ancestries for three main reasons, detailed below.

First, SNP-level GWA results are difficult to interpret across human ancestries due to a litany of confounding variables. For a given phenotype, SNP-level GWA summary statistics may differ across ancestries due to a variety of factors, including: $(i)$ ascertainment bias in genotyping 2 , $(i i)$ varying linkage disequilibrium (LD) patterns ${ }^{12 \mid 13}$, ( iii) variation in allele frequencies due to different selective pressures from unique population histories $\frac{13}{17}$, and $(i v)$ the effect of environmental factors on phenotypic variation $\frac{18}{21}$. These confounders and the observed low transferability of GWA results across ancestries 222 have generated an important call for increasing GWA efforts focused on diverse, non-European ancestry samples. However, we note that non-European ancestry GWA studies have — and will continue to have — smaller sample sizes than existing and emerging European-ancestry GWA cohorts, reducing the precision of summary statistic estimation in these studies. Focusing energy and resources to increasing GWA sample sizes without intentional focus on sampling of non-European populations will thus likely perpetuate an already troubling history of leaving non-European ancestry samples out of GWA studies of large-scale biobanks such as the UK Biobank ${ }^{23}$ (but see Sinnott-Armstrong et al. ${ }^{\sqrt[24]{3}}$ ). What has received less attention is the potential of new and existing methods, in conjunction with increased sampling efforts, to characterize genetic trait architecture using multi-ancestry datasets while accounting for variable statistical power to detect, estimate, and replicate genetic associations across cohorts.

The second reason that GWA studies have failed to characterize complex trait architecture across diverse human ancestries is that the GWA SNP-level test of association is rarely applied to non-European ancestry 
samples 25 . Even when diverse ancestries are sampled, GWA studies usually condition on GWA results identified using European ancestry cohorts to detect other SNP-level associations ${ }^{6}$. There are two possible explanations for leaving non-European ancestry samples out of analyses: $(i)$ researchers are electing to not analyze diverse cohorts due to a lack of statistical power and concerns over other confounding variables (recently covered in Ben-Eghan et al. ${ }^{[23}$ ); or (ii) the analyses of non-European cohorts yield no genome-wide significant SNP-level associations. In either case, valuable information is being ignored in GWA studies or going unreported in resulting publications $2325 \mid 26$. In our own analysis of abstracts of publications between 2012 and 2020 using UK Biobank data, we found that only 33 out of 166 studies (19.87\%) reported genomewide significant associations in any non-European ancestry cohort (see Online Materials and Methods and Supplementary Figure 1- Supplementary Figure 2,

The final reason GWA studies do not fully characterize multi-ancestry genetic trait architecture is the false assumption that, given enough samples, the additive effect of individual mutations can sufficiently explain genetic trait architecture 27 . There is undoubted benefit from increased sampling for both association mapping and heritability estimation, but the downstream consequences of deeper sampling in essentially one human ancestry have received little attention. Increased sampling of non-European ancestry cohorts may lead to discovery of novel and ancestry-specific associations and improve power to estimate broad and narrow-sense heritability. Many recent methodological advances that leverage GWA summary statistics have focused on: testing the co-localization of causal SNPs (e.g., fine mapping 28 [30); the non-additive effects of SNP-level interactions (i.e., epistasis $\frac{\sqrt{3132}}{3}$ ); and multivariate GWA tests $32 \sqrt[35]{35}$. While these methods can be extended and applied to multi-ancestry GWA analyses, they still focus on SNP-level signals of genetic trait architecture (see also Brown et al. ${ }^{\sqrt[36]{6}}$, Galinsky et al. .37 ). Unlike the traditional GWA method, enrichment analyses increase statistical power by aggregating SNP-level signals of genetic associations while allowing for genetic heterogeneity in trait architecture across samples and offer the opportunity for immediate insights into trait architecture using existing datasets. However, these methods have been comparatively overlooked in multi-ancestry GWA mapping studies and heritability estimation.

To showcase the opportunities for insight into genetic trait architecture from enrichment analyses across genomic scales and diverse human ancestries, we present a new framework for genetic association mapping that tests SNPs, genes, and pathways in multiple ancestry groups for associations with a trait of interest. This analysis requires the generation of no additional information than what is required for (paired genotype and phenotype data) or generated as output from (effect sizes, standard errors, $p$-values) standard GWA analysis. Moving beyond SNP-level associations allows for more comprehensive probing of shared and ancestry-specific mechanisms underlying genetic trait architecture. We illustrate insights gained from this multiscale framework using 25 quantitative traits and more than 600,000 diverse individuals from the UK 
Biobank, BioBank Japan, and the PAGE study data (Supplementary Table 1- Supplementary Table 9).

\section{Replication of SNP-level GWA results across ancestries is the ex- ception, not the rule}

One question that has not been interrogated fully is the extent to which SNP-level associations for a given trait replicate across ancestries (however, also see Wojcik et al. $\underline{6}$, Durvasula and Lohmueller ${ }^{22}$, Carlson

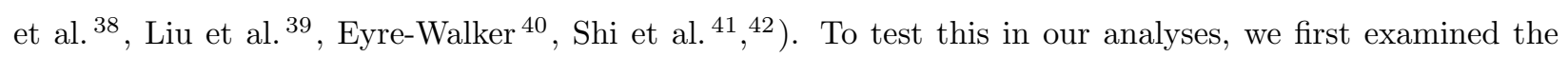
number of genome-wide significant SNP-level associations that replicated exactly based on chromosomal position in multiple ancestries (see Supplementary Figure 3 and Supplementary Figure 3., with Bonferronicorrected thresholds provided in Supplementary Table 10. Exact replication of at least one SNP-level association across two or more ancestries occurs in all 25 traits we studied. The C-reactive protein (CRP) trait had the highest proportion of replicated results, with $18.95 \%$ replicating in at least two ancestries, but has a relatively low number of unique GWA significant SNPs $(2,734)$ when compared to other traits (Figure 1). This is likely because the genetic architecture of CRP is sparse and highly conserved across ancestries (Figure 2). We note that the concordance of genome-wide significant SNP-level association statistics for CRP among five ancestry cohorts is exceptional. In the other 24 traits we analyzed, we did not observe the same number of SNP-level replications among five cohorts. C-reactive protein, which is encoded by the gene of the same name located on chromosome 1, is synthesized in the liver and released into the bloodstream in response to inflammation. In our standard GWA analysis of SNP-level association signal in each ancestry cohort with CRP, rs3091244 is genome-wide significant in a single ancestry cohort. rs3091244 has been functionally validated as influencing C-reactive protein levels $\frac{43[44}{4}$ and is linked to genome-wide significant SNPs in the other two ancestries for which genotype data is available. Interestingly, all GWA significant SNPlevel associations for CRP in the Native Hawaiian ancestry cohort replicate in both the African-American (PAGE) and the Hispanic and Latin American cohorts (all use the same genotyping array). We did not observe high levels of GWA significant SNP-level replication in other analyzed traits — suggesting that CRP is the exception, not the rule. In 23 traits, the proportion of genome-wide SNP-level replications was below 10\% (Figure 1a). For polygenic traits, replication of SNP-level mapping results is challenging to interpret considering the large number of GWA significant associations for the trait overall. For example, height contains the largest number of replicated SNP-level associations in our multi-ancestry analysis — but these only represent $8.90 \%$ of all unique SNP-level associations with height discovered in any ancestry cohort. A more comprehensive list of previously associated variants is available for both height and CRP in the 
Supplemental Note.

Often, replication across ancestries is tested using genomic regions centered on a SNP. Scans across the region surrounding the SNP of interest are usually defined arbitrarily — using physical windows (or "clumps") to smooth over ascertainment bias and varying LD across ancestries instead of using regions that are biologically motivated such as genes or transcriptional elements. While clumping presents an easy way to scan for regional replication of a given GWA finding, its results are not readily interpretable. We also performed clumping using windows of size $1 \mathrm{Mb}$ centered around significant SNP-level associations (see Online Materials and Methods). Height had the largest proportion of windows that contain a SNP-level association that replicated in at least two ancestries (Supplementary Figure 3b and Supplementary Figure 3e). In the three traits with the greatest proportion of windows containing SNP-level replications - height $(77.09 \%$ of clumps), urate (65.89\%), and low density lipoprotein (54.40\%) — we then recorded the number of genes and transcriptional elements within the window that contained GWA significant SNP-level associations. We found that for all three traits, the vast majority of $1 \mathrm{Mb}$ windows that were used to clump SNP-level associations contained multiple genes and transcriptional elements with significantly associated SNPs: height (94.04\%, 17.93 genes in clump (mean) \pm 15.71 (standard deviation)), urate $(97.47 \%, 18.44 \pm 13.72$ ), and low density lipoprotein $(99.12 \%, 14.85 \pm 12.89)$. The clumping procedure obscures the true genetic trait architecture by preventing localization of true biological mechanism and coarsely conflating genomic proximity with shared genetic contribution. Thus, we find window-based clumping does not easily produce biologically motivated hypotheses for downstream validation. Recent multi-ancestry GWA studies and methods have tested for evidence of effect size heterogeneity among different ancestry cohorts ${ }_{6 / 28|45| 46}$. We applied the fine-mapping method $\mathrm{SuSiE}^{46}$ to study signals of effect size heterogeneity in the three ancestry cohorts for which we had access to genotype data (UK Biobank European ancestry, African ancestry, and South Asian ancestry individuals; see Supplementary Table 1). We find little evidence of correlated effect sizes among ancestry cohorts, including among independent subsamples of the UK Biobank European ancestry individuals Supplementary Table 11- Supplementary Table 13.

\section{Valuing biological mechanism over statistical significance}

Enrichment analyses aggregate SNP-level association statistics using predefined SNP sets, genes, and pathway annotations to identify regions of the genome enriched for trait associations beyond what is expected by chance. Published enrichment analyses have demonstrated the ability to identify trait associations that go unidentified when using the standard SNP-level GWA analysis alone $47 \mid 53$. The standard GWA method is known to have a high false discovery rate $(\mathrm{FDR})^{54 / 55}$. One benefit of enrichment analyses is that they 
mitigate this inflation of statistical association signal. Supplementary Figure 8 and Supplementary Figure 9 illustrate that both regression with summary statistics (RSS) ${ }^{52}$, a fully Bayesian method, and our method gene- $\varepsilon^{53}$ control FDR well both in the presence and absence of population structure. Enrichment methods also enable identifying biologically interpretable trait associations in studies with smaller sample sizes than emerging GWA studies. For example, Nakka et al. 56 identified an association between ST3GAL3 and attention deficit hyperactivity disorder (ADHD) using methods that aggregated SNP-level signals across genes and networks. ADHD is a trait with heritability estimates as high as $75 \%$ which had no known genome-wide significant SNP-level associations at the time; Nakka et al. ${ }^{[56}$ studied genotype data from just 3,319 individuals with cases, 2,455 controls and 2,064 trios $\frac{57}{57}$. A study by Demontis et al. $\frac{58}{58}$ later found a SNP-level association in the ST3GAL3 gene, but was only able to do so with a cohort an order of magnitude larger (20,183 individuals diagnosed with ADHD and 35,191 controls, totaling 55,374 individuals).

Because non-European GWA ancestry cohorts usually have much smaller sample sizes compared to studies with individuals of European ancestry, enrichment analyses should be standard practice to boost statistical power and identify biologically relevant genetic associations with traits of interest. In an analysis performed by Ben-Eghan et al. $\stackrel{23}{23}$ on 45 studies analyzing UK Biobank data, the second most commonly stated reason for omitting non-European cohorts in any one study was due to lack of power. We urge researchers to note that enrichment analyses model linkage disequilibrium, allow for genetic heterogeneity in trait architecture, and are an immediately available and biologically interpretable way to test for associations in cohorts that are considered under-powered in a standard GWA analysis. Furthermore, if a GWA study has been performed then researchers have all of the necessary information available (summary statistics and genotype data) to apply these methods (along with ancestry-matched LD matrices); failure to do so will only serve to reinforce the growing disparity in genetic association analyses.

We tested for gene-level associations in each of the 25 complex traits in each ancestry cohort for which we had data (Supplementary Table 1 - Supplementary Table 9), and identified associations in genes and transcriptional elements shared across ancestries for every trait. All of our analyses used our method gene$\varepsilon$ : an empirical Bayesian approach that reformulates the GWA SNP-level null hypothesis to distinguish between $(i)$ mutations that are statistically associated with the disease but are unlikely to directly influence it, and (ii) mutations that are most strongly associated with a disease of interest. The gene- $\varepsilon$ gene-level test statistic is a quadratic form aggregating SNP-level GWA summary statistics, where $p$-values for each gene are derived by constructing an empirical null distribution based on the eigenvalues of a gene-specific partitioning of the LD matrix (for more details, see Cheng et al.53). Our analyses show that several hematological traits have a higher rate of significant gene-level associations that replicate across multiple ancestry cohorts than SNP-level associations that replicate across ancestry cohorts (Figure 1b). These include, platelet 
count (PLC), mean corpuscular hemoglobin (MCH), mean corpuscular hemoglobin concentration (MCHC), hematocrit, hemoglobin, mean corpuscular volume (MCV), red blood cell count (RBC), and neutrophil count (Supplementary Figure 3). Focusing on platelet count as an example, we identify 65 genes that are [significantly enriched for associations in multiple ancestries when tested using gene- $\varepsilon$ (see Supplementary Table 14 for details on Bonferroni thresholds used to correct for the number of genes tested) $\frac{53}{5}$. Fifty-five of these genes are significantly associated in both the European and East Asian ancestry cohorts, and the remaining ten all replicate in other pairs of ancestry cohorts. Overall, each of the six ancestry cohorts in pur analysis shares at least one significant gene with another ancestry cohort, as shown in Supplementary Figure 10,

Results from gene-level enrichment analyses can be further propagated on protein-protein interaction networks to identify interacting genes enriched for association through known networks 59 . Often, studies use network propagation as a way to incorporate information from multiple "omics" databases in order to identify significantly mutated gene subnetworks or modules contributing to a particular disease ${ }^{60}$. An unexplored extension of network propagation is how it can be used with GWA results to identify significantly mutated subnetworks that are shared or ancestry-specific in multi-ancestry GWA datasets.

To explore network propagation of gene-level association results in our analyses, we applied the Hierarchical HotNet method ${ }^{61}$ to gene- $\varepsilon$ gene-level association statistics for each trait-ancestry data set. Here, we focus on network analysis of triglyceride levels in six ancestry cohorts: African-American (PAGE), European, East Asian, South Asian, Hispanic and Latin American, and Native Hawaiian. Figure 3 shows our findings at the network level in the European, East Asian, and Native Hawaiian cohorts (networks separated by ancestry are available in Supplementary Figure 11. In both the European and East Asian cohorts, we identify enrichment of mutations in a highly connected subnetwork of genes in the apolipoprotein family. In addition, we identify a gene network enriched for mutations in the East Asian and Native Hawaiian cohorts that also interact with the subnetwork enriched for mutations in both the European and East Asian cohorts. For instance, beta-secretase 1 (BACE1), is a genome-wide significant gene-level association in the East Asian cohort but contains no previously associated SNPs with triglycerides in any ancestry cohort in the GWAS catalog. Additionally, both APOL1 and HBA1 were identified as significantly associated with triglycerides using gene- $\varepsilon$ in our analysis of the Native Hawaiian ancestry cohort, and both genes were part of significant subnetworks identified by Hierarchical HotNet in the European and Native Hawaiian ancestry cohorts. Detailed replication of SNP-level and gene-level associations among ancestries for triglyceride levels are shown in Supplementary Figure 12 and Supplementary Figure 13 , respectively.

We find widespread evidence of heterogeneous replication of results across genomic scales (i.e., genes classified as significantly mutated in cohort A are not classified as such in cohort B but do contain significant 
SNP-level associations in cohort B; see Supplementary Table 16. These contrasting association results at different genomic scales are likely due to differences in LD and effect size estimates, underscoring that association test results beyond the SNP-level are more easily interpretable across ancestries. Enrichment analyses provide more power to detect genetic architecture and more work should be done to develop methods that can unify insight into the genetic basis of complex traits across genomic scales. SNP-level and genelevel association results are discussed in more detail for both platelet count and triglyceride levels in the Supplemental Note.

\section{Bridging association and heritability}

Estimation of narrow-sense heritability for complex traits remains a central goal in medical and population genetics. Over the past five years, multiple methodological advances have shown that the inclusion of the covariance between the effect sizes (i.e., an LD matrix) derived in a GWA analysis can vastly improve performance in heritability estimation from unrelated individuals ${ }^{62} 66$. We focused our heritability analyses on two questions. First, given that heritability estimates are region-based statistics, are regions explaining a large proportion of total trait heritability also significantly associated with the same phenotype? Second, should we expect regions that make large genetic contributions to heritability in one cohort to generalize across ancestry cohorts?

To investigate these questions, we obtained heritability estimates using Heritability Estimator from Summary Statistics (HESS) $)^{66}$ for the 25 quantitative traits we studied in the European, South Asian, and African ancestry cohorts from the UK Biobank as well as the East Asian ancestry cohort from the Biobank Japan. In the two largest ancestry cohorts studied - the UK Biobank European and Biobank Japan East Asian ancestry cohorts - we partitioned the regional heritability estimated by chromosome. We used these estimates to identify genomic regions that make significantly large contributions to the narrow-sense heritability in the same trait in both cohorts, as shown in Figure 4a. We further focus on urate levels as a model trait for exploring these questions, extending results observed by Sinnott-Armstrong et al. ${ }^{67}$ to multiple ancestry cohorts and different datasets. For example, in our analyses, a single region on chromosome 4 explains a total of $86.83 \%$ and $32.34 \%$ of the observed heritability in urate levels in the European and East Asian cohorts, respectively. In the European ancestry cohort, there are 880 genome-wide significant SNPs within the region (chromosome 4: 9,217,084-10,698,980) and 2,261 genome-wide significant SNPs within the analogous genomic region in the East Asian cohort (chromosome 4: 9,326,479-10,699,152), corresponding to large amounts of heritability explained as shown in Figure $4 \mathrm{p}$-c. In a similar analysis of the South Asian ancestry cohort, HESS identifies the same region on chromosome 4 as explaining a large amount of the narrow-sense 
heritability levels, but no other regions made significant contributions. The African ancestry cohort is notably too small $(N=4,967)$ to gain enough statistical power to identify enriched regions for contributions to narrow-sense heritability and is thus not included here (see Supplementary Figure 14a-b).

In these heritability analyses with HESS, we used an ancestry-specific set of regional boundaries that represent approximately independent LD blocks ${ }^{66 / 68}$; this is in contrast to gene-level enrichment analyses, that do not focus on approximately independent LD blocks. This means gene-level enrichment analyses may create autocorrelated test statistics due to overlapping gene boundaries in a given region. Heritability estimation is simply a linear combination of SNP-level effect sizes; it is crucial to note that gene-level enrichment methods like gene- $\varepsilon^{\frac{53}{3}}$ aggregate effect size estimates across a set of SNPs and test the aggregated statistic against null distributions based on the LD structure in a specified genomic region. For example, while the region on chromosome 4 that contains the SLC2A9 gene explains the largest proportion of heritability in both ancestry cohorts, there are only 2 genome-wide significant genes in the European ancestry cohort and 7 significant genes in the East Asian ancestry cohort. Our results in Supplementary Figure 3p and f suggest that, while the same genomic regions are often enriched for contributions to narrow-sense heritability across ancestry cohorts, ancestry-specific variation in LD maps influences the detection of significantly associated genes across ancestry cohorts.

The region on chromosome 4 that explains the greatest amount of heritability in both the European and East Asian ancestry cohorts contains a portion of the transport gene-solute carrier family 2, facilitated glucose transporter member 9 (SLC2A9) - which plays a critical role in the urate biosynthesis and transport pathway. This region was previously identified as enriched for contributions to heritability in the UK Biobank European ancestry cohort 67 .

In addition to the region surrounding $S L C 2 A 9$, we identify three more regions explaining a large proportion of heritability that are detected in both the East Asian and European ancestry cohorts. These three regions each contain genes known to play a role in the urate biosynthesis pathway and have been previously shown to contain GWA association with urate levels: Glucokinase regulatory protein (GCKR on chromosome $\left.2^{67 / 69}\right)$, ATP binding cassette subfamily G member 2 ( $A B C G$ on chromosome $4^{70}$ ), and solute carrier family 22 member 11 (SLC22A11 on chromosome 1171). We note that no previous study has identified these regions with significant contributions to narrow-sense heritability in multiple ancestry cohorts.

In our analyses, the European and East Asian ancestry cohorts each have an ancestry-specific region explaining a significant amount of heritability. In the European ancestry cohort, a region containing solute carrier family 17 member 1 (SLC17A1) on chromosome 6 explains a significant amount of heritability and contains SNPs that have been previously associated with gout ${ }^{72}$. In the East Asian ancestry cohort, a region containing the solute carrier family 16 member 9 (SLC16A9) on chromosome 10 explains a significant 
amount of the heritability of urate levels, and it has been previously associated with the development of gout in an East Asian ancestry cohort 73 .

Our analysis of urate levels in three ancestry cohorts prioritizes a set of regions that contribute to heritability in multiple ancestry cohorts. Of the four genes that we highlight in these shared regions, three (SLC2A9, SLC22A11, and ABCG2) were significantly associated with urate levels in our gene-level association analyses in both the European and East Asian ancestry cohorts (see Data Availability). We additionally identify regions in the European and East Asian ancestry cohorts enriched for heritability contributing SNPs, thereby offering potential novel and ancestry-specific candidates for the genetic architecture of urate levels.

The results of our association and heritability analyses for multiple complex traits generate a new array of questions. For most traits, we observe heterogeneity between association signals and heritability estimates. Specifically, the regional associations statistics obtained using gene- $\varepsilon$ and the regional heritability estimates obtained using the HESS method $\sqrt{66}$ do not necessarily identify the same regions for the same trait as significant. Given the disjoint set of results from the two analyses, it is not immediately clear how to interpret these differences between statistical association and narrow-sense heritability estimates. Fundamentally, association studies are a question of variable selection in statistics; meaning that the goal is to identify the correct explanatory variables as significant and determine the rank-order of their strength of association is secondary 7476 . On the other hand, accurate narrow-sense heritability estimation is reliant upon obtaining the correct combination of weights on each SNP such that their linear combination is representative of the proportion of phenotypic variance explained by a particular region. It is still unknown why the distribution of heritability across SNPs does not correspond to statistically significant SNPs in the same region. Effect size heterogeneity across ancestries could additionally exacerbate these differences Supplementary Table 11 - Supplementary Table 13). An important area of future work will be to interrogate the relationship between regions enriched for heritability contributions with the variable selection done in SNP-level and gene-level association tests. Confidence intervals for SNP-level effect size estimates will undoubtedly be crucial in connecting these two concepts $\sqrt{55}$ for downstream analyses like functional validation and phenotype prediction.

\section{Complex traits demand complex methods}

As has been previously noted $[\sqrt[5 \mid 23]{ }$, non-European ancestry cohorts are often excluded from GWA analyses of multi-ancestry biobanks. Unless this practice is curbed by the biomedical research community, it will exacerbate already existing disparities in healthcare across diverse communities. In this perspective, complementary to but distinct from recent work focusing on proposing changes to multi-ancestry GWA study 
design 2 215/6122 $26 \mid 77 / 78$ we have focused on the potential of association-based methods to increase the information gained from multi-ancestry GWA datasets and particularly to generate hypotheses about multi-ancestry genetic trait architecture at biologically interpretable genomic scales. We demonstrate the potential gains of moving beyond standard SNP-level GWA analysis using of 25 quantitative complex traits among eight diverse human cohorts in two large biobanks - BioBank Japan and the UK Biobank, and the PAGE consortium database Supplementary Table 1- Supplementary Table 9). We believe the potential contribution of methods to more equitable genomic analyses for diverse humans has been overlooked and is a crucial step towards realizing the promise of personalized medicine.

Two open questions must be tackled when studying complex trait architecture in the multi-ancestry biobank era: $(i)$ to what extent is the true genetic trait architecture heterogeneous among ancestry groups? 6 [6/79 and (ii) are GWA results (both the SNPs classified as statistically associated and their estimated effect sizes) transferable across ancestries, at any genomic scale? Continued applications of the standard SNP-level GWA approach will not answer these questions. However, many methods that aggregate SNP-level effects, test for effect size heterogeneity, leverage genomic annotations and gene interaction networks offer opportunities to directly test these fundamental questions. Such approaches have yet to be combined with studies of how evolutionary processes play a role in shaping the genetic architecture of complex traits, although the evolution of human complex trait architecture has been the subject of recent scrutiny $20140|80| 81$. While many studies note that differences in LD across ancestries affects transferability of effect size estimates 6 , in population genetics have additionally debated how various selection pressures and genetic drift may hamper transferability of GWA results across ancestries (see for example, Edge and Rosenberg ${ }^{15,16}$, Novembre and Barton ${ }^{18}$, Harpak and Przeworski ${ }^{20}$, Durvasula and Lohmueller ${ }^{22}$ ).

In this perspective, we have not addressed the downstream consequences of using self-identified ancestry to define cohorts in biobank sized GWA studies (but see Urbut et al. ${ }^{35}$, Willer et al. $\stackrel{85}{ }$, Lin et al. $\frac{86}{}$, Yang et al. .87$)$. Each sample we studied here has also experienced environmental exposures that may influence the statistical detection of genetic associations, and some of those environmental exposures may be correlated with genomic ancestry 13188 90. Interrogation of the influence of gene by environment interactions on complex traits must be done with highly controlled experiments, which can in turn help prioritize traits in which association studies will be interpretable and useful. Increasing sample size in GWA studies alone will not resolve these fundamental biological questions: the proportion of phenotypic variance explained by associations discovered as sample sizes increase in GWA studies has already reached diminishing returns ${ }^{91}$, and gene by environmental interactions are increasingly influential in large biobanks with cryptic relatedness ${ }^{92}$.

Although the genomic era is producing incredible datasets whose dimensions were unimaginable even a decade ago, biobanks continue to be largely composed of European ancestry individuals. Biomedical 
researchers should continue to pressure both funding agencies and institutions to diversify their sampling efforts in the name of inclusion and addressing - instead of exacerbating - genomic health disparities. In addition to those efforts, we believe existing and new methods can increase the return on investment in multiancestry biobanks, ensure that every bit of information from these datasets is studied, and prioritize biological mechanism above SNP-level statistical association signals. Methods can and should play an important role as biomedical research shifts current paradigms to extend the benefits of personalized medicine beyond people of European ancestry.

\section{Data Availability}

All scripts, publicly available data, and outputs from GWA, gene, and pathway association tests are available at https://github.com/smithsap/redefining_replication. This repository also holds heritability estimates for each trait using ancestry cohorts from the UK Biobank and Biobank Japan.

\section{Acknowledgments}

We thank Kirk Lohmueller and Alicia R. Martin for helpful comments on an earlier version of this manuscript, as well as the Crawford and Ramachandran Labs for helpful discussions. This research was conducted in part using computational resources and services at the Center for Computation and Visualization at Brown University as well as, using the UK Biobank Resource under Application Number 22419. The Population Architecture Using Genomics and Epidemiology (PAGE) program is funded by the National Human Genome Research Institute (NHGRI) with co-funding from the National Institute on Minority Health and Health Disparities (NIMHD). The WHI program is funded by the National Heart, Lung, and Blood Institute, National Institutes of Health, U.S. Department of Health and Human Services through contracts 75N92021D00001, 75N92021D00002, 75N92021D00003, 75N92021D00004, 75N92021D00005. The HCHS/SOL study was carried out as a collaborative study supported by contracts from the National Heart, Lung and Blood Institute (NHLBI) to the University of North Carolina (N01-HC65233), University of Miami (N01-HC65234), Albert Einstein College of Medicine (N01-HC65235), Northwestern University (N01-HC65236) and San Diego State University (N01-HC65237). S.P.S. is a trainee supported under the Brown University Predoctoral Training Program in Biological Data Science (NIH T32 GM128596). L.C. acknowledges the support of an Alfred P. Sloan Research Fellowship, and a David \& Lucile Packard Fellowship for Science and Engineering. This work was also supported by US National Institutes of Health R01 GM118652 to S.R., and S.R. acknowledges additional support from National Science Foundation CAREER Award DBI-1452622. 


\section{Figures and Tables}

\begin{tabular}{|c|c|c|c|}
\hline Genomic Scale & Association Test & $\begin{array}{c}\text { Model of Genetic Trait } \\
\text { Architecture }\end{array}$ & Relevant Example \\
\hline SNPs & $\begin{array}{c}\text { Standard univariate } \\
\text { genome-wide association } \\
\text { (GWA) test }\end{array}$ & $\begin{array}{c}\text { The true mutation-level trait } \\
\text { architecture is the same for all } \\
\text { individuals. }\end{array}$ & $\begin{array}{c}\text { Many inflammatory bowel disease } \\
\text { mutations replicate across ancestries }\end{array}$ \\
\hline SNP-Sets/Genes & $\begin{array}{c}\text { Gene-level association } \\
\text { tests (e.g., gene- } \varepsilon^{53} \\
\text { SKAT }\end{array}$ & $\begin{array}{c}\text { Core genes are the same across } \\
\text { all ancestries, with potentially } \\
\text { varying causal SNPs. }\end{array}$ & $\begin{array}{c}\text { Late-onset Alzheimer's disease risk } \\
\text { from ApoE4 allele is lower in African } \\
\text { ancestry individuals }\end{array}$ \\
\hline Pathways/Networks
\end{tabular}

Table 1: The three genomic scales and corresponding association tests used in this study. The models of genetic trait architecture corresponding to each genomic scale and statistical method that have been previously invoked in the literature (including relevant examples cited in the last column). We argue these nested genomic scales should routinely be leveraged in multi-ancestry GWA studies to generate biologically interpretable characterization of trait architecture across ancestries. 
a

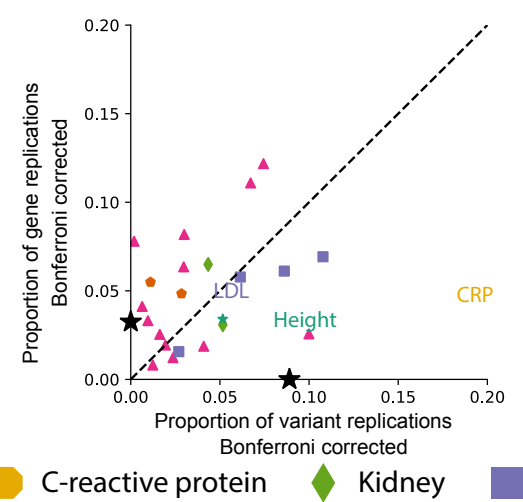

b

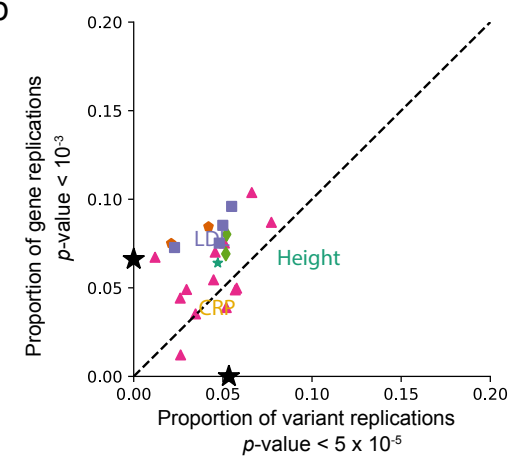

Metabolic c

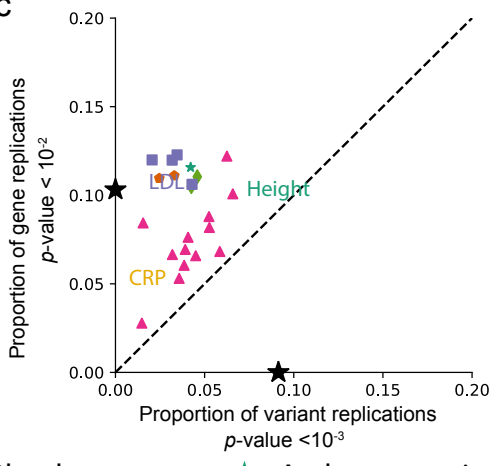

Blood pressure

$\star$ Anthropometric

Figure 1: Less stringent significance thresholds lead to a decrease in the proportion of replicated SNP-level associations and an increase in the proportion of gene-level associations among ancestries for each of the $\mathbf{2 5}$ traits analyzed. a. Proportion of all SNP-level Bonferroni-corrected genome-wide significant associations in any ancestry that replicate in at least one other ancestry is shown on the x-axis (see Supplementary Table 10 for ancestry-trait specific Bonferroni corrected $p$-value thresholds). On the y-axis we show the proportion of significant gene-level associations that were replicated for a given phenotype in at least two ancestries (see Supplementary Table 14 for Bonferroni corrected significance thresholds for each ancestry-trait pair). The black stars on the $\mathrm{x}$ - and $\mathrm{y}$-axes represent the mean proportion of replicates in SNP and gene analyses, respectively. C-reactive protein (CRP) contains the greatest proportion of replicated SNP-level associations of any of the phenotypes. $\mathbf{b}$. The $\mathrm{x}$-axis indicates the proportion of SNP-level associations that surpass a nominal threshold of $p$-value $<10^{-5}$ in at least one ancestry cohort that replicate in at least one other ancestry cohort. The y-axis indicates the proportion of gene-level associations that surpass a nominal threshold of $p$-value $<10^{-3}$ in at least one ancestry cohort and replicate in at least one other ancestry cohort. Nominal $p$-value thresholds tend to decrease the proportion of replicated SNP-level associations and tend to increase the proportion of replicated gene-level associations. The number of unique SNPs and genes that replicated in each cohort is given in Supplementary Figure 5. c. The x-axis indicates the proportion of SNP-level associations that surpass a nominal threshold of $p$-value $<10^{-3}$ in at least one ancestry cohort that replicate in at least one other ancestry cohort. The $\mathrm{y}$-axis indicates the proportion of gene-level associations that surpass a nominal threshold of $p$-value $<10^{-2}$ in at least one ancestry cohort and replicate in at least one other ancestry cohort. The number of unique SNPs and genes that replicated in each cohort is given in Supplementary Figure 6 As shown in panel b, nominal $p$-value thresholds tend to decrease the proportion of replicated SNP-level associations and tend to increase the proportion of replicated gene-level associations. Expansion of three letter trait codes are given in Supplementary Table 2. Supplementary Figure 4 shows the same set of plots with all traits represented as text. 
bioRxiv preprint doi: https://doi.org/10.1101/2021.04.20.440612; this version posted April 20, 2021. The copyright holder for this preprint (which was not certified by peer review) is the author/funder, who has granted bioRxiv a license to display the preprint in perpetuity. It is made available under aCC-BY-NC-ND 4.0 International license.
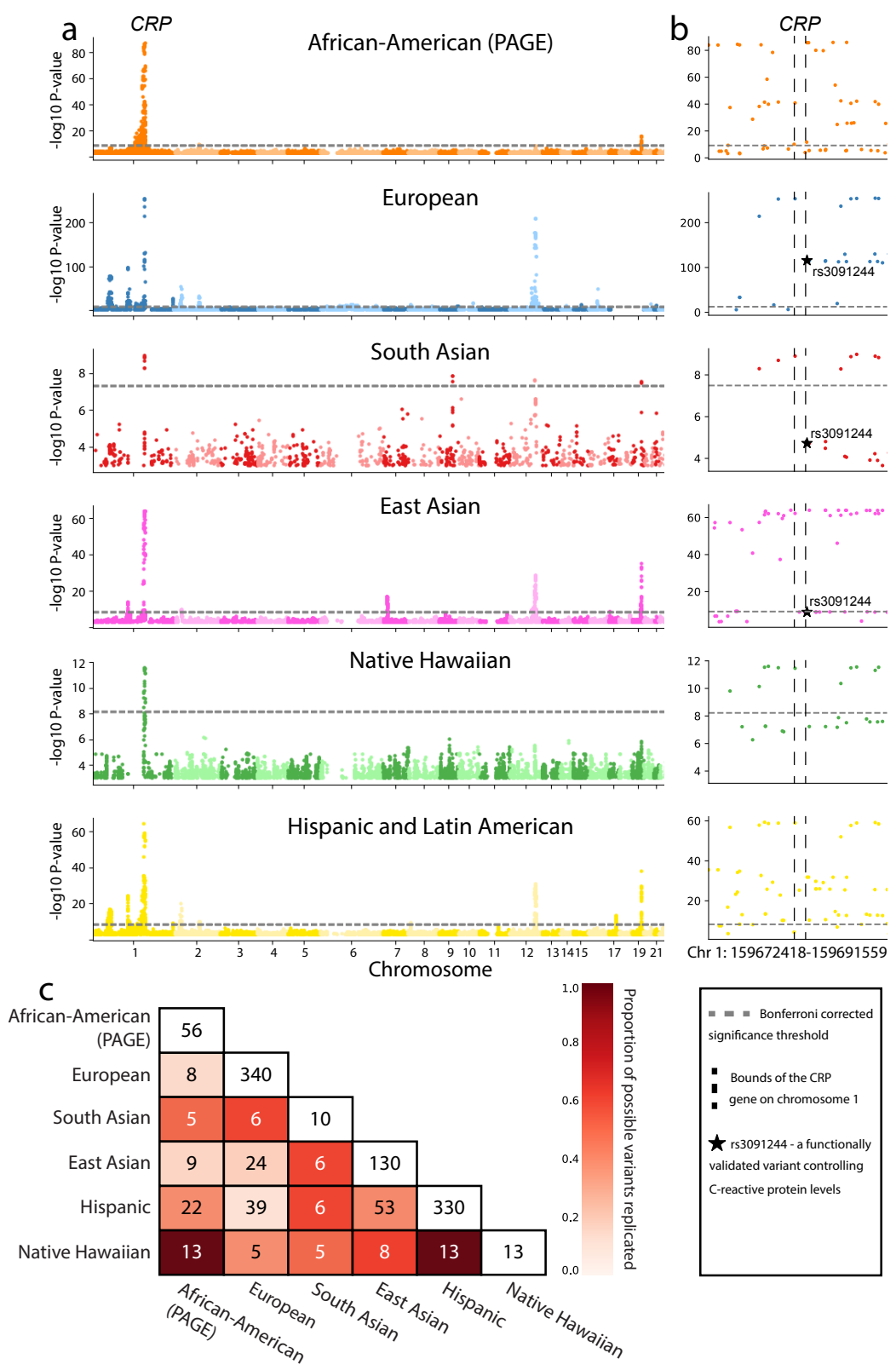

Figure 2: C-reactive protein is an exceptional trait where standard GWA analyses may be sufficient to identify shared genetic architecture among ancestry cohorts. a. Genome-wide Manhattan plot for C-reactive protein levels. Ancestry-specific Bonferroni-corrected significance thresholds are shown with dashed horizontal grey lines and listed in Supplementary Table 10. Note that the scale of the $-\log _{10^{-}}$transformed $p$-values on the y-axis is different for each ancestry for clarity. b. Manhattan plot of SNP-level associations around the $C R P$ gene located on chromosome 1 for each ancestry (zoomed in from panel a). Boundaries of the $C R P$ gene are shown with vertical dashed black lines. All six ancestries contain genome-wide significant SNPs in the region. Black stars in the European, South Asian, and East Asian plots represent rs3091244, a SNP that has been functionally validated as contributing to serum levels of C-reactive protein ${ }^{43144}$. c. Heatmap of Bonferroni-corrected significant genotyped SNPs replicated between each pair of ancestries analyzed. Here, we focus on SNPs in the $1 \mathrm{MB}$ region surrounding the CRP gene. Entries along the diagonal represent the total number of SNP-level associations in the $1 \mathrm{MB}$ region surrounding the $C R P$ gene for each ancestry. The color of each cell is proportional to the percentage of SNP-level associations replicated out of all possible replications in each ancestry pair (i.e., the minimum of the diagonal entries between the pairs being considered). For example, the maximum number of genome-wide significant SNPs that can possibly replicate between the Hispanic and East Asian is 25, and 20 replicate resulting in the cell color denoting $80 \%$ replication. A similar matrix, computed including imputed SNPs is shown in Supplementary Figure 7 


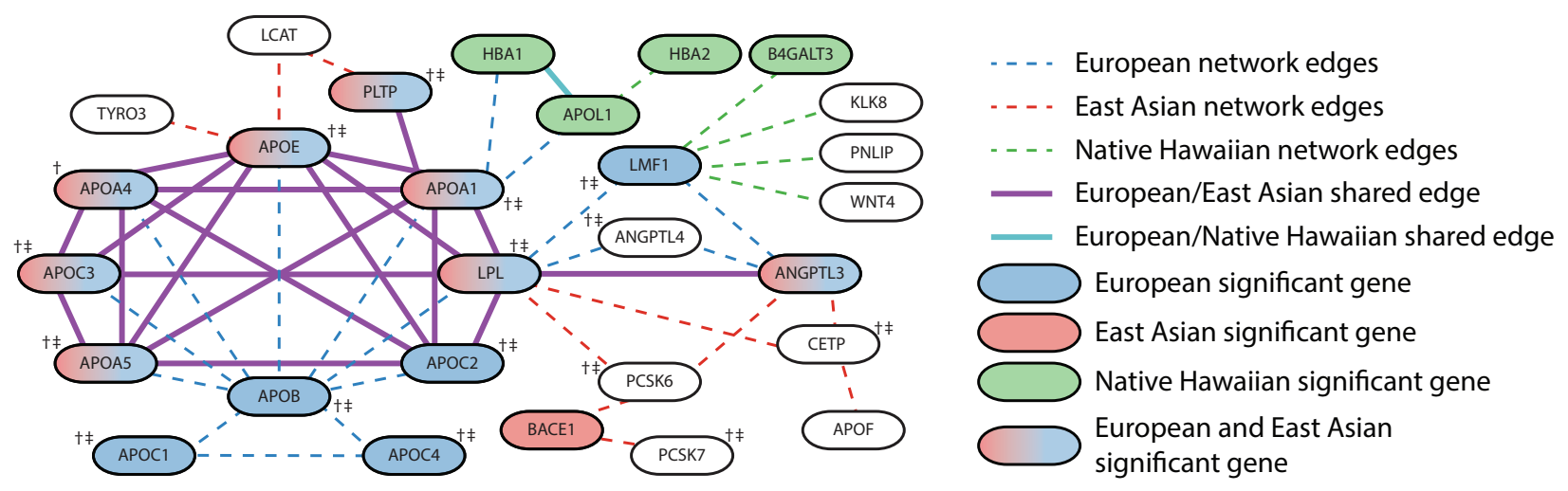

Figure 3: A subnetwork of apolipoprotein genes is significantly enriched for mutations in European, East Asian, and Native Hawaiian ancestries associated with triglyceride levels. The largest significantly altered subnetwork $(p$-value $<0.05)$ for triglyceride levels contains overlapping gene subnetworks for each of the European, East Asian, and Native Hawaiian ancestries when analyzed independently with Hierarchical HotNet $\underline{61}$. Each node in the network represents a gene. The shading of each node indicates the statistical significance of the association of that gene with triglyceride levels in a particular cohort. Two genes are connected if their protein products interact based on the ReactomeFI $2016^{94}$ (European, East Asian) or iRefIndex 15.0 ${ }^{95}$ (Native Hawaiian) protein-protein interaction networks. Several genes from the apolipoprotein gene family are significantly associated with triglyceride levels in both the European and East Asian cohorts (see Data Availability). Additionally, the interactions between them form a highly connected subnetwork. Smaller subnetworks identified in the Native Hawaiian cohort are distal modules that are connected to the subnetwork detected in the European cohort. Not all genes in the largest significantly altered subnetwork for the Native Hawaiian ancestry group are shown for visualization purposes (127 not pictured here). Genes that contain SNPs previously associated to triglyceride levels in a European cohort in the GWAS catalog are indicated with $\dagger$. Similarly, genes that contain SNPs previously associated with triglyceride levels in a non-European cohort in the GWAS Catalog are indicated with $\ddagger$. The studies identifying these associations are given in Supplementary Table 15. 

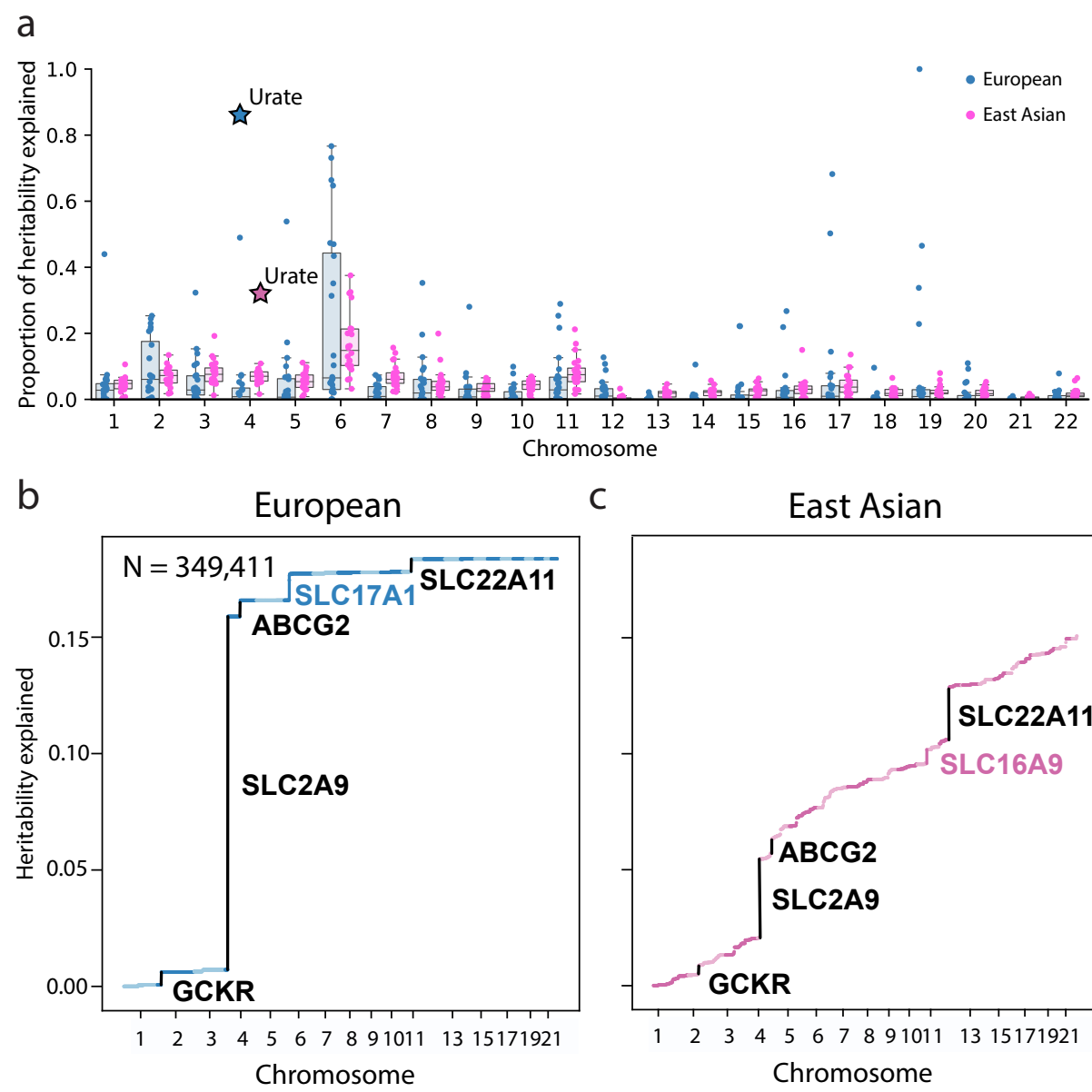

C East Asian

d
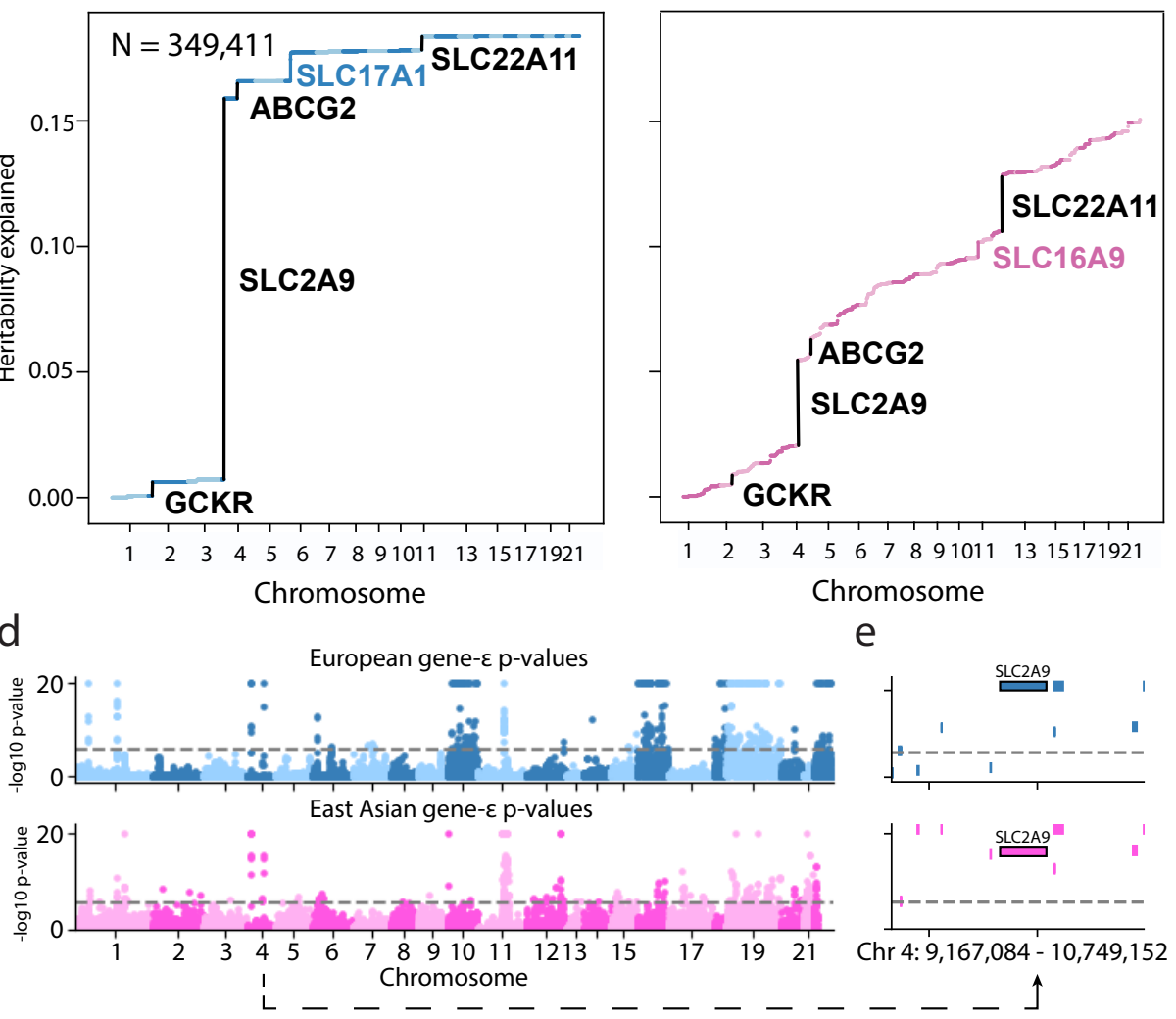

Figure 4: Heritability estimation and gene-level association statistics do not categorically identify different genomic regions underwriting urate levels. a. Proportion of narrow-sense heritability explained by each chromosome for the 25 traits analyzed in the European and East Asian ancestries. For each of the 25 trait,s we obtained estimates of heritability explained by each chromosome using HESS 6 . For each chromosome and each of the two ancestry cohorts, we summarize proportion of heritability explained for 25 traits using boxplots (see also Figure 4 in Shi et al.66). b-c. Regions enriched for SNPs contributing to narrow-sense heritability of urate levels are shared across (b) European and (c) East Asian ancestries. Estimates for each ancestry were obtained using HESS ${ }^{66}$. Four regions across the genome make large contributions to the narrow-sense heritability of urate levels, and are found in both the European and East Asian ancestries. One of these, containing known urate transporter SLC2A9, is also identified in our analysis of the South Asian ancestry cohort (Supplementary Figure 14). Additionally, both the European and East Asian ancestries have regions contributing to narrow-sense heritability that are not identified in the other; these contain known urate transporters SLC17A1 and SLC16A9 (labeled using ancestry-specific colors), respectively. d. Genome-wide Manhattan plot of gene-level $p$-values for urate levels in the European and East Asian ancestry cohorts calculated using the gene- $\varepsilon$ method ${ }^{53}$. e. Local gene-level Manhattan plot of the region explaining the greatest amount of narrow-sense heritability of urate levels in both the European and East Asian ancestry cohorts located on chromosome 4. In each cohort, SLC2A9 surpasses the genome-wide Bonferroni-corrected significance threshold in gene-level analysis using gene- $\varepsilon$ and lies within the region explaining the greatest amount of narrow-sense heritability. 


\section{Online Materials and Methods}

\section{Data overview}

We performed statistical tests of association at the SNP, gene, and pathway-level for 25 quantitative traits. These analyses were performed on seven ancestry cohorts drawn from UK Biobank, BioBank Japan, and PAGE consortia (Supplementary Table 3). Descriptions of the samples studied, including sample size, and number of SNPs for each ancestry cohort are given in Supplementary Table 1 and Supplementary Table 4 Supplementary Table 9.

\section{UK Biobank Data}

[We downloaded individual genotype data using the UK Biobank's (UKB) ukbgene resource, https:// biobank.ctsu.ox.ac.uk/crystal/download.cgi. European individuals from the UK Biobank data were selected using the self-identified ancestry (data field 21000) using values outlined at https://biobank. ctsu.ox.ac.uk/crystal/field.cgi?id=21000. Using the relatedness file provided by the UK Biobank, one individual from each related pair was then randomly removed. This process was repeated for individuals whose self-identified ancestry was South Asian.

We performed unsupervised genome-wide ancestry estimation using ADMIXTURE by setting $K=3^{96}$ on the self-identified African ancestry cohort. We also included YRI and CEU individuals in the ADMIXTURE runs from the 1000 Genomes Project, to identify the ancestry components corresponding to African and European ancestry. We removed individuals containing less than 5\% membership in the African ancestry component and more than 5\% membership in the third component, which corresponds to American Indian/Alaskan Native (AIAN) ancestry (Supplementary Figure 15). We downloaded imputed SNP data from the UK Biobank for all remaining individuals and removed SNPs with an information score below 0.8. Information scores for each SNP are provided by the UK Biobank (http://biobank.ctsu.ox.ac.uk/ crystal/refer.cgi?id=1967). The remaining genotype and high-quality imputed SNPs were put through a stringent quality control pipeline in each ancestry cohort to obtain cohort-specific SNPs to be used for further analysis as detailed in the main text (detailed below).

We performed the following quality control filters in the European, South Asian, and African cohorts from the UK Biobank (Application number 22419). Genotype data for 488,377 individuals in the UK Biobank were downloaded using the UK Biobank's ukbgene (https://biobank.ctsu.ox.ac.uk/crystal/ download.cgi) tool and converted using the provided ukbconv tool (https://biobank.ctsu.ox.ac.uk/ crystal/refer.cgi?id=149660). Phenotype data was also downloaded for those same individuals using 
the ukbgene tool. Individuals identified by the UK Biobank to have high heterozygosity, excessive relatedness, or aneuploidy were removed (1,550 individuals). After then separating individuals into self-identified ancestral groups using data field 21000. Within these cohorts, unrelated individuals were then selected by randomly selecting an individual from each pair of related individuals. This resulted in 349,469 European individuals, 5,716 South Asian individuals, and 4,967 African individuals.

Genotype quality control was then performed on each cohort separately using the following steps. All structural variants were first removed, leaving only single nucleotide polymorphisms in the genotype data. Next, all AT/CG SNPs were removed to avoid possible confounding due to sequencing errors. Then, SNPs with minor allele frequency less than $1 \%$ were removed using the plink $2^{97}$--maf 0.01 . We then removed all SNPs found to be in Hardy-Weinberg equilibrium, using the plink --hwe 0.000001 flag to remove all variants with a Fisher's exact test $p$-value $>10^{-6}$. Finally, all SNPs with missingness greater than $1 \%$ were removed using the plink --mind 0.01 flag.

\section{Biobank Japan Data}

We downloaded summary statistics for 25 quantitative traits from the Biobank Japan (BBJ) website (http: $/ /$ jenger.riken.jp/en/result) 198 100 . The sample descriptions and number of SNPs included in our analyses are given in Supplementary Table 4. The number of SNPs included in the analysis of each trait represent those SNPs that: $(i)$ contained an rsid number that could be mapped to the hg19 genome build, (ii) overlapped with SNPs contained within the 1000 Genomes Project phase 3 genotype data, and (iii) had a minor allele frequency greater than 0.01 in Japanese (JPT) individuals in the 1000 Genomes Project. We used the 1000 Genomes phase 3 data from 93 JPT individuals to estimate the linkage disequilibrium (LD) between SNPs in BioBank Japan for which we had the summary statistic data; LD was estimated separately for each of the 25 quantitative traits using the trait specific SNP arrays. LD estimates were used in the calculation of regional association statistics.

\section{Population Architecture using Genomics and Epidemiology (PAGE) Study Data}

Summary statistics for genotyped and imputed SNPs in five admixed populations were downloaded from the Population Architecture using Genomics and Epidemiology (PAGE) ${ }^{6}$ with permission granted via approval of manuscript proposal. We included summary statistics for up to 14 quantitative traits for African-American, Hispanic and Latin American, Native Hawaiian, American Indian/Alaska Native, and Asian ancestry cohorts when available. All AT/CG SNPs were omitted, and SNPs with an IMPUTE2 information score greater than 0.8 were included in this analysis. Number of individuals and SNPs varied across ancestry-trait combinations 
and are given in Supplementary Table 4- Supplementary Table 9.

Individuals from the 1000 Genomes Project phase $3^{101}$ and the Human Genome Diversity Panel (HGDP) 102 were used to obtain LD estimates between SNPs for each ancestry cohort. To construct the LD reference panel for PAGE summary statistics from the African-American ancestry cohort, unrelated individuals from the 1000 Genomes Americans of African Ancestry in SW USA (ASW) and African Caribbeans in Barbados (ACB) were used. Only SNPs found in both the 1000 Genomes imputed data and PAGE summary statistics files were used in gene-level association and heritability analyses. We used the same approach to compute reference LD estimates between SNPs for the Hispanic and Latin American, AIAN, and Asian ancestry cohorts, with the following 1000 Genomes reference population, respectively: Mexican Ancestry from Los Angeles USA (MXL) and Puerto Ricans from Puerto Rico (PUR); Colombians from Medellin, Colombia (CLM) and Peruvians from Lima, Peru (PEL); and the East Asian superpopulation (EAS). For the Native Hawaiian individuals from the PAGE study, there were no appropriate reference populations in the 1000 Genomes data. In order to construct a reference LD matrix for the Native Hawaiian ancestry cohort, we randomly sampled individuals from populations in the most recent release of the HGDP proportional to the global ancestry of the Native Hawaiian cohort. The Native Hawaiian cohort's global ancestry proportions were determined using ADMIXTURE runs to be 47.89\% Oceanian, 25.16\% East Asian, 25.51\% European, $0.90 \%$ African, and $0.54 \%$ AIAN in a separate publication (Wojcik preprint - in prep.). We did not sample from populations with less than $1 \%$ of the total ancestry in the admixture analysis referenced above. The resulting sample from which LD was estimated included 39 individuals from the Papuan Sepik in New Guinea and Melanesian in Bougainville, 14 individuals from the French in France, and 14 individuals from the Yoruba in Nigeria.

\section{SNP-level GWA analyses}

In the European, African, and South Asian ancestrty cohorts from the UK Biobank, we performed GWA studies for each ancestry-trait pair in order to test whether the same $\operatorname{SNP}(\mathrm{s})$ are associated with a given quantitative trait in different ancestries. SNP-level GWA effect sizes were calculated using plink and the --glm flag97. Age, sex, and the first ten principal components were included as covariates for all traits analyzed. Principal components analysis was performed using flashpca $2.0^{103}$ on a set of independent markers derived separately for each ancestry cohort using the plink --indep-pairwise 100100.1 command. Summary statistics for the 25 quantitative traits in the Biobank Japan, as well as available ancestry-trait pairs in the PAGE data were then compared with the results from the association analyses in the UK Biobank cohorts (same traits as listed in Supplementary Table 4). In each analysis of an ancestry-trait pair, 
a separate Bonferonni-corrected significance threshold was calculated using the number of SNPs tested in that particular ancestry-trait pair (Supplementary Table 10).

To perform SNP-level fine mapping analyses on a given quantitative trait, we applied Sum of Single Effects ( $\mathrm{SuSiE})$ variable selection software? ${ }^{\text {? }}$ SuSiE implements a Bayesian linear regression model on individual level data where sparse prior distributions are placed on the effect size of SNP and posterior inclusion probabilities (PIPs) are used to summarize their statistical relevance to the trait of interest. The software for $\mathrm{SuSiE}$ requires an input $\ell$ which fixes the maximum number of causal SNPs to include in the model. In this work, we consider results when this parameter is chosen conservatively $(\ell=3000)$. We used the three cohorts for which we had genotype data from the UK Biobank (African, European, and South Asian) to test whether there was effect size heterogeneity among ancestries in the 25 traits analyzed in this study. We first selected ten independent, non-overlapping subsamples of 10,000 individuals from the European ancestry cohort and filtered out any variants that had a minor allele frequency of less than 0.01. For each subsample, we then applied SuSiE to each of the 25 traits and compared the effect sizes and posterior inclusion probabilities. The average number of SNPs with an effect size greater than 0.01 and average number of SNPs with a PIP greater than 0.01 for each trait across the ten cohorts are reported in Supplementary Table 11. Supplementary Table 11 also reports the median correlation coefficient of effect sizes and PIPs among the 45 pairwise comparisons between the 10 subsample cohorts.

We then applied SuSiE to the African and South Asian ancestry cohorts and compared their resulting effect sizes and PIPs to ten independent, non-overlapping subsamples of the European ancestry cohort. The number of SNPs with an effect size greater than 0.1 and PIPs greater than 0.01 in both the focal cohort (either African or South Asian) and at least one of the ten European ancestry subsamples of the same size are reported in Supplementary Table 12 and Supplementary Table 13. Also reported in these tables, are the mean number of effect sizes greater than 0.01 and PIPs greater than 0.01 across the European ancestry subsamples for each trait and the number of unique effect sizes greater than 0.01 and PIPs greater than 0.01 that were only identified in the African or South Asian ancestry cohorts. Finally, Supplementary Table 12 and Supplementary Table 13report the median correlation coefficient of the African or South Asian ancestry cohort effect sizes and PIPs with the ten European ancestry subsample cohorts of the same size.

\section{Gene-level association tests}

In order to test aggregated sets of SNP-level GWA effect sizes for enrichment of associated mutations with a given quantitative trait, we applied gene- $\varepsilon^{53}$ to each ancestry cohort for each trait of interest. The gene- $\varepsilon$ method takes two summary statistics as inputs: (i) SNP-level GWA marginal effect size estimates $\hat{\boldsymbol{\beta}}$ derived 
using ordinary least squares and (ii) an LD matrix $\boldsymbol{\Sigma}$ empirically estimated from external data (e.g., directly from GWA study genotype data, or using an existing LD map from a population with similar genomic ancestry to that of the samples analyzed in the GWA study). It is well-known that SNP-level effect size estimates can be inflated due to various correlation structures among genome-wide genotypes. Thus as a first step, gene- $\varepsilon$ uses its inputs to derive regularized effect size estimates $\tilde{\boldsymbol{\beta}}$ through elastic net penalized regression. A unique feature of gene- $\varepsilon$ is that it treats SNPs with spuriously associated nonzero effects as nonassociated. gene- $\varepsilon$ assumes a reformulated null distribution of SNP-level effects $\tilde{\beta}_{j} \sim \mathcal{N}\left(0, \sigma_{\varepsilon}^{2}\right)$, where $\sigma_{\varepsilon}^{2}$ is the SNP-level null threshold and represents the maximum proportion of phenotypic variance explained (PVE) by a spurious or non-associated SNP. This leads to the reformulated SNP-level null hypothesis $H_{0}: \mathbb{E}\left[\beta_{j}^{2}\right] \leq \sigma_{\varepsilon}^{2}$. To infer an appropriate $\sigma_{\varepsilon}^{2}$, gene- $\varepsilon$ fits a $K$-mixture of normal distributions over the regularized effect sizes with successively smaller variances (i.e., $\sigma_{1}^{2}>\cdots>\sigma_{K}^{2}=0$ ). In this study as in Cheng et al.53, we assume that associated SNPs will appear in the first set, while spurious and non-associated SNPs appear in the latter sets. As a final step, gene- $\varepsilon$ computes its gene-level association test statistic for the $g$-th gene by conformably partitioning the regularized GWA effect size estimates and computing the quadratic form $\tilde{Q}_{g}=\tilde{\boldsymbol{\beta}}_{g}^{\top} \tilde{\boldsymbol{\beta}}_{g}$. Corresponding $p$-values are then derived using Imhof's method. This assumes the common genelevel null $H_{0}: Q_{g}=0$, where the null distribution of $Q_{g}$ is dependent upon the eigenvalues from the scaled LD matrix $\sigma_{\varepsilon}^{2} \boldsymbol{\Sigma}$. For details on implementation, validation and performance comparison with simulations, and empirical application to UK Biobank white British individuals in six traits, see Cheng et al.53.

In practice, gene- $\varepsilon$ and other enrichment analyses ${ }^{48 / 56191}$ can be applied to any user-specified set of genomic regions, such as regulatory elements, intergenic regions, or gene sets. These gene-level association tests enable identifying traits in which genetic trait architecture involves the same set of core genes across individuals and may be heterogeneous among individuals at the SNP-level. gene- $\varepsilon$ takes as input a list of boundaries for all regions to be analyzed. In our study, we applied gene- $\varepsilon$ to all genes and transcriptional elements defined in Gusev et al. 104 for human genome build 19. Throughout this study, we refer to the resulting effect size estimates produced by gene- $\varepsilon$ as "gene-level association statistics".

In our gene-level analysis, SNP arrays included both genotyped and imputed SNPs with an information score $>0.8$ for each ancestry-trait pair. To compute the LD matrix, we first pruned highly linked SNPs so that the number of SNPs included for any chromosome was less than 35,000 SNPs - the computational limit of gene- $\varepsilon$ - using the plink command --indep-pairwise 10010 0.5. For the UK Biobank European, South Asian, and African ancestry cohorts, we then derived empirical LD estimates between each pair of SNPs for each chromosome in each cohort using plink's --r square command applied to the empirical genotype and high confidence imputed data, defined as having an information score as greater than or equal to 0.8. The ancestry-specific SNP arrays were used to calculate gene-level association statistics for each 
ancestry, including: 23,603 gene-level association statistics for the European ancestry cohort, 23,671 genelevel association statistics for the South Asian ancestry cohort, and 23,575 gene-level association statistics for the African ancestry cohort.

To calculate gene-level association statistics using Biobank Japan summary statistics, we first found the intersection between SNPs included in the analysis of each trait (Supplementary Table 4) and SNPs included in the 1000 Genomes Project phase 3 data for the sample of 93 JPT individuals. Note, this intersection was different among some traits as the genotpye data in the Biobank Japan were from different studies, which in turn used different genotyping arrays. We then pruned highly linked markers for each trait separately using the plink flag --indep-pairwise $10010 \mathrm{X}$ where $\mathrm{X}$ was determined by finding the highest possible value that led to less than 35,000 SNPs being included on each chromosome for the trait. Because of the increased density of SNPs with effect size estimates for Height, $\mathrm{X}$ was set to prune more conservatively at $\mathrm{X}=0.15$. For all other traits, $\mathrm{X}$ was set to 0.5 . The number of regions for which a gene- $\varepsilon$ gene-level association statistic was calculated for each trait is given in Supplementary Table 4

GWA summary statistics for the five cohorts in the PAGE data set were used as input to gene- $\varepsilon$ for each available ancestry-trait combination. The array of markers for each ancestry cohort in the PAGE study data was pruned using plink and the --indep-pairwise $10010 \mathrm{X}$ flag. $\mathrm{X}$ was set to the maximum value in each ancestry that ensured no chromosome contained more than 35,000 markers: $\mathrm{X}$ was set to 0.05 for the African-American cohort, 0.08 for the Hispanic and Latin American and AIAN cohorts, and 0.25 for the Asian and Native Hawaiian cohorts.

Finally, for each ancestry-trait combination, genes that passed the Bonferroni corrected $p$-value $(p=$ $0.05 /$ number of genes tested) were labeled as "significant" throughout this study.

\section{Pathway analysis and network propagation using Hierarchical HotNet}

We tested for shared and divergent enrichment of association with a trait of interest at the gene-level among interacting genes using network propagation of gene- $\varepsilon$ gene-level association statistics as input to Hierarchical HotNet $\underline{61}$. Hierarchical HotNet identifies significantly altered subnetworks using gene-level scores as input; in this study, these gene scores were set to $-\log _{10}$-transformed $p$-values of gene- $\varepsilon$ gene-level association test statistics (see also Nakka et al. $\frac{56,105}{10}$ ). For each ancestry-trait combination, we assigned $p$-values of 1 to genes with $p$-values greater than 0.1 to make the resulting networks both sparse and more interpretable (again see Nakka et al.56|105). In addition, ancestry-trait pairs in which less than 25 genes produced gene- $\varepsilon$ $p$-values less than 0.1 were discarded.

We used three protein-protein interaction networks: ReactomeFI 2016 ${ }^{94}$, iRefIndex 15.0 ${ }^{95}$, and HINT+HI $106 \mid 107$. 
For the ReactomeFI 2016 interaction network, interactions with confidence scores less than 0.75 were discarded. The HINT+HI interaction network consists of the combination of all interactions in HINT binary, HINT co-complex, and HuRI HI interaction networks. We ran Hierarchical HotNet (10 $0^{3}$ permutations) on the thresholded $-\log _{10}$-transformed gene-level $p$-values for each ancestry-trait combination. We restricted our analysis to the largest subnetwork identified in each significant ancestry-trait-interaction network combination $(p<0.05)$.

\section{Heritability estimation using HESS}

We obtained SNP-level narrow-sense heritability estimates for each ancestry-trait combination using the Heritability Estimator from Summary Statistics (HESS) ${ }^{66}$. For the European ancestry cohort in the UK Biobank, we applied the ashr $\sqrt{55}$ in order to correct for inflated GWA effect size estimates.

HESS requires partition files, which define approximately independent genomic regions in each cohort. Appropriate partition files are essential to accurately estimate SNP-level narrow-sense heritability as too many blocks will lead to inflated estimates. For the European (UKB), African (UKB), African-American (PAGE), East Asian (BBJ), and South Asian (UKB) ancestry cohorts, we used partition files from Berisa and Pickrell 68 . We set any resulting negative regional heritability estimates from HESS to zero, and calculated the total narrow-sense heritability for each ancestry-trait pair as the sum of non-negative heritability estimates across the genome. We calculated regional heritability estimates for the regions specified in each ancestry cohort's partition file across ancestries for the same traits to identify regions enriched for hertabilitycontributing SNPs across multiple ancestry cohorts.

\section{Clustering traits sharing a core set of associated genes using the WINGS algo- rithm}

We used the WINGS algorithm $\frac{108}{10}$ to identify clusters of traits sharing a core set of genes enriched for associated mutations. WINGS takes as input a gene $(M)$ by trait $(N)$ matrix and uses the Ward distance metric to find the distance among vectors of gene scores for different phenotypes; in this study, we used gene- $\varepsilon$ gene-level association statistics as the input to WINGS. The more significantly associated genes that two traits share, the closer they will be in the gene-dimensional space. Applying WINGS to a matrix of gene scores for each ancestry separately, we examined whether the same traits clustered together, separately in each ancestry. We constructed matrices of gene- $\varepsilon$ gene-level association statistics for the UK Biobank European, African, South Asian (from the UK Biobank) and East Asian (Biobank Japan) ancestry cohorts. Each of these matrices contained gene-level association statistics for all 25 quantitative traits of interest. 
The total number of genes and regulatory regions included were: European (23,603), African (23,575), South Asian(23,671), and East Asian (21,435). For the East Asian ancestry cohort, we limited the genes to the intersection of genes with gene- $\varepsilon$ gene-level association statistics across all 25 traits. The number of gene scores calculated for each trait in the East Asian ancestry cohort varies due to the heterogeneity in imputed and genotype SNP arrays in the Biobank Japan studies Supplementary Table 4 and Supplementary Table 14. Supplementary Figure 16 shows the resulting dendrograms displaying prioritized phenotypes identified using the WINGS algorithm on each cohort's gene score matrix. The WINGS algorithm is designed to run on 25 phenotypes or more (see McGuirl et al. 108 for deatils), and we therefore did not apply the WINGS algorithm to the AIAN, Native Hawaiian, or Hispanic and Latin American cohorts as there was not data for enough phenotypes (Supplementary Table 5 Supplementary Table 9).

\section{Analysis of GWAS Catalog Metadata and Previous GWA Publications}

We cross-referenced our results from association testing at multiple genomic scales against previously published results in the GWAS catalog (https://www.ebi.ac.uk/gwas/) and in PubMed using the following processes.

In order to collect PubMed IDs (PMIDs) for publications associated with the UK Biobank, a two-part data collection process was used. The first process was to directly search for publications with variations of the term "UK Biobank" (e.g., U.K. Biobank, United Kingdom Biobank) from PubMed using the Entrez Programming Utilities (E-Utilities) API. The E-Utilities API is the public API to the NCBI Entrez system and allows direct access to all Entrez databases including PubMed. Search queries were formulated by narrowing publications using year published and then further narrowing to those publications with variations of the search term "UK Biobank" in either the title or abstract. The open-source Python package Entrez (https://biopython.org/DIST/docs/api/Bio.Entrez-module.html) from the Biopython Project was used to facilitate interaction with the E-Utilities API.

The second data collection process was to gather information from publications listed directly on the UK Biobank website (https://www.ukbiobank.ac.uk/). Since the majority of publications on the website did not have an easily accessible PMID, identifying information including publication title and year was scraped and used to retrieve a publication's corresponding PMID (again using the E-Utilities API). The HTML/XML document parsing Python library Beautiful Soup (https://www.crummy.com/software/BeautifulSoup/ bs4/doc/) was used to parse the HTML of the various UK Biobank webpages, and the Python Requests library (https://requests.readthedocs.io/en/master/) was used to programatically send HTTP calls to the server hosting the website. PMIDs were retrieved directly from the XML output of the E-Utilities 
API calls.

The PMIDs retrieved from both processes were aggregated into a single set of unique PMIDs, as some publications were identified by both processes. Publications that could not get associated PMIDs from the second data collection process were flagged for manual processing. The PMIDs that were retrieved from PubMed directly but could not be found based on the publication information provided on the UK Biobank website were noted. Conversely, the PMIDs that could be retrieved from publication information found on the UK Biobank website but not directly from PubMed were also noted.

Using the compiled list of PMIDs, analyses of the UK Biobank data set reported in the GWAS catalog association data were compiled. Previous genotype-to-phenotype association data and sample ancestry descriptions were downloaded from https://www.ebi.ac.uk/gwas/docs/file-downloads. Unique genotype-to-phenotype associations were parsed using a set of custom python scripts. All scripts used in the curation of PMIDs, parsing of GWAS catalog summary data, and determination of previously published genotype-to-phenotype associations from UK Biobank studies are available on GitHub (https: //github.com/ramachandran-lab/redefining_replication).

\section{Simulation design to test the power and false discovery rate of GWA and gene- level association analyses}

In our simulation studies, we used the following general simulation scheme to generate quantitative traits using real genotype data on chromosome 1 from $N$ randomly sampled individuals of European ancestry in the UK Biobank. This pipeline follows from previous studies 3153 . We will use $\mathbf{X}$ to denote the $N \times J$ genotype matrix, with $J$ denoting the number of single nucleotide polymorphisms (SNPs) encoded as 0,1,2 copies of a reference allele at each locus and $\mathbf{x}_{j}$ representing the genotypic vector for the $j$-th SNP. Following quality control procedures detailed in the Online Methods, our simulations included $J=36,518$ SNPs distributed across genome. We used the NCBI's RefSeq database in the UCSC Genome Browser to assign SNPs to genes which resulted in $G=1,408$ genes in the simulation studies.

After the annotation step, we simulated phenotypes by first assuming that the total phenotypic variance $\mathbb{V}[\mathbf{y}]=1$, and that all observed genetic effects explained a fixed proportion of this value (i.e., narrow-sense heritability, $h^{2}$ ). Next, we randomly selected a certain percentage of genes to be enriched fotr associations and denoted the sets of SNPs that they contained as $\mathcal{C}$. Within $\mathcal{C}$, we selected causal SNPs in a way such that each associated gene at least contains one SNP with non-zero effect size. Quantitative continuous traits were then generated under the following two general linear models:

- Standard Model: $\mathbf{y}=\sum_{c \in \mathcal{C}} \mathbf{x}_{c} \beta_{c}+\mathbf{e}$ 
- Population Structure Model: $\mathbf{y}=\mathbf{W b}+\sum_{c \in \mathcal{C}} \mathbf{x}_{c} \beta_{c}+\mathbf{e}$

where $\mathbf{y}$ is an $N$-dimensional vector containing all the phenotype states; $\mathbf{x}_{c}$ is the genotype for the $c$-th causal SNP; $\beta_{c}$ is the additive effect size for the $c$-th SNP; and $\mathbf{e} \sim \mathcal{N}\left(0, \tau^{2} \mathbf{I}\right)$ is an $N$-dimensional vector of normally distributed environmental noise. Additionally, in the model with population structure, $\mathbf{W}$ is an $N \times M$ matrix of the top $M=10$ principal components (PCs) from the genotype matrix and represents additional population structure with corresponding fixed effects $\mathbf{b}$. The effect sizes of SNPs in genes enriched for associations are randomly drawn from standard normal distributions and then rescaled so they explain a fixed proportion of the narrow-sense heritability $\mathbb{V}\left[\sum \mathbf{x}_{c} \beta_{c}\right]=h^{2}$. The coefficients for the genotype PCs are also drawn from standard normal distributions and rescaled such that $\mathbb{V}[\mathbf{W b}]=10 \%$ of the total phenotypic variance, with the variance of all non-genetic effects contributing $\mathbb{V}[\mathbf{W b}]+\mathbb{V}[\mathbf{e}]=\left(1-h^{2}\right)$. For any simulations conducted under the population structure model, genotype PCs are not included in any of the model fitting procedures, and no other preprocessing normalizations were carried out to account for the additional population structure. More specifically, GWA summary statistics are then computed by fitting a single-SNP univariate linear model via ordinary least squares (OLS):

$$
\widehat{\beta}_{j}=\left(\mathbf{x}_{j}^{\top} \mathbf{x}_{j}\right)^{-1} \mathbf{x}_{j}^{\top} \mathbf{y}
$$

for every SNP in the data $j=1, \ldots J$. These OLS effect size estimates, along with an empirically LD matrix $\boldsymbol{\Sigma}$ computed directly from the full $N \times J$ genotype matrix $\mathbf{X}$, are given to gene- $\varepsilon$. We also retain standard errors and $p$-values for the implementation of competing methods: RSS ${ }^{52}$, SKAT 48 , and the standard GWA SNP-level association test. Given the simulation procedure above, we simulate a wide range of scenarios for comparing the performance of gene-level association approaches by varying the following parameters:

- Number of individuals: $N=5,000$ and 10,000;

- Narrow-sense heritability: $h^{2}=0.2$ and 0.6 ;

- Percentage of enriched genes: $1 \%$ (sparse) and 10\% (polygenic);

Furthermore, we set the number of causal SNPs with non-zero effects to be some fixed percentage of all SNPs located within the designated genes enriched for associations. We set this percentage to be $0.125 \%$ in the $1 \%$ associated SNP-set case, and 3\% in the 10\% associated SNP-set case. All performance comparisons are based on 100 different simulated runs for each parameter combination. Lastly, for each simulated dataset, we also selected some number of intergenic SNPs (i.e., SNPs not mapped to any gene) to have non-zero effect sizes. This was done to mimic genetic associations in unannotated regulatory elements. Specifically, 
five randomly selected intergenic SNPs were given non-zero contributions to the trait heritability in the $1 \%$ enriched genes case, and 30 intergenic SNPs were selected in the $10 \%$ enriched genes case.

All performance comparisons are based on 100 different simulated runs for each parameter combination. We computed gene-level $p$-values for gene- $\varepsilon$, SKAT, and the single-SNP GWAS. For evaluating the performance of RSS, we compute posterior enrichment probabilities. For all approaches, we assessed the power and false discovery rates when identifying enriched genes at either a Bonferroni-corrected threshold $\left(p=0.05 / 1,408\right.$ genes $\left.=3.55 \times 10^{-5}\right)$ or according to the median probability model (posterior enrichment probability $>0.5)^{109}$. Supplementary Figure 8 and Supplementary Figure 9 show the mean performances (and standard errors) across all simulated replicates. 
bioRxiv preprint doi: https://doi.org/10.1101/2021.04.20.440612; this version posted April 20, 2021. The copyright holder for this preprint (which was not certified by peer review) is the author/funder, who has granted bioRxiv a license to display the preprint in perpetuity. It is made available under aCC-BY-NC-ND 4.0 International license.

\section{${ }_{700}$ Supplementary Tables}


bioRxiv preprint doi: https://doi.org/10.1101/2021.04.20.440612; this version posted April 20, 2021. The copyright holder for this preprint (which was not certified by peer review) is the author/funder, who has granted bioRxiv a license to display the preprint in perpetuity. It is made available under aCC-BY-NC-ND 4.0 International license.

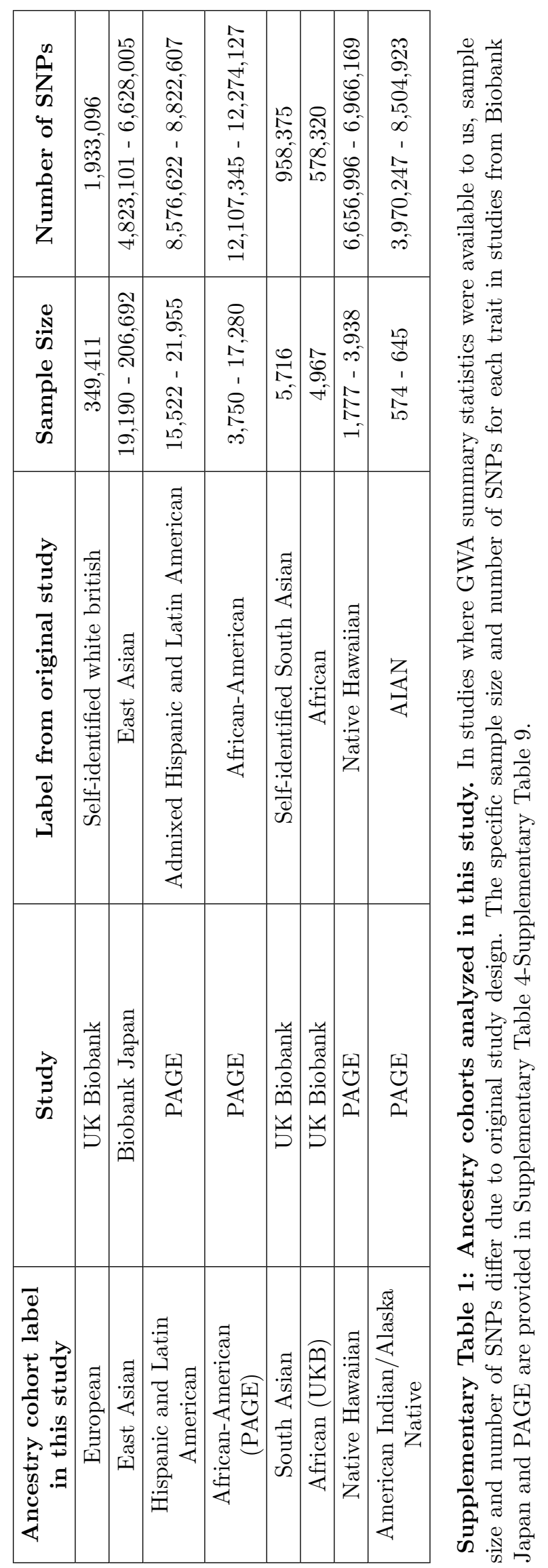




\begin{tabular}{|c|c|}
\hline Trait Name & Code \\
\hline Body mass index & BMI \\
\hline High density lipoprotein & HDL \\
\hline Low density lipoprotein & LDL \\
\hline Hemoglobin A1c & HBA1C \\
\hline Estimated glomerular filtration rate & EGFR \\
\hline C-reactive protein & CRP \\
\hline Systolic blood pressure & SBP \\
\hline Diastolic blood pressure & DBP \\
\hline Platelet count & PLC \\
\hline Mean corpuscular hemoglobin concentration & MCHC \\
\hline Mean corpuscular hemoglobin & MCH \\
\hline Mean corpuscular volume & MCV \\
\hline Red blood cell count & RBC \\
\hline White blood cell count & WBC \\
\hline
\end{tabular}

Supplementary Table 2: Abbreviations used throughout this study for 14 quantitative traits analyzed in this study. The remaining 11 traits analyzed were Basiphil count, Cholesterol, Eosinophil count, Height, Hematocrit, Hemoglobin, Lymphocyte count, Monocyte count, Neutrophil count, and Triglyceride levels, respectively. These are not abbreviated in the main text. 


\begin{tabular}{|c|c|c|c|}
\hline Trait Name or Code & AIAN & Native Hawaiian & Hispanic \\
\hline BMI & Yes & Yes & Yes \\
\hline Basophil count & No & No & No \\
\hline CRP & Yes & Yes & Yes \\
\hline Cholesterol & Yes & Yes & Yes \\
\hline DBP & Yes & No & Yes \\
\hline EGFR & Yes & No & Yes \\
\hline Eosinophil count & No & No & No \\
\hline HBA1C & Yes & Yes & Yes \\
\hline HDL & Yes & Yes & Yes \\
\hline Height & Yes & Yes & Yes \\
\hline Hematocrit & No & No & No \\
\hline Hemoglobin & No & No & No \\
\hline LDL & Yes & Yes & Yes \\
\hline Lymphocyte count & No & No & No \\
\hline MCH & Yes & No & Yes \\
\hline MCHC & No & No & No \\
\hline MCV & No & No & No \\
\hline Monocyte count & No & No & No \\
\hline Neutrophil count & No & No & No \\
\hline PLC & Yes & No & Yes \\
\hline RBC & No & No & No \\
\hline SBP & Yes & No & Yes \\
\hline Triglyceride & No & Yes & Yes \\
\hline Urate & No & No & No \\
\hline WBC & Yes & & Nos \\
\hline
\end{tabular}

Supplementary Table 3: Traits assayed in the PAGE study data by ancestry cohort. Data were available for each of the 25 listed traits in the UK Biobank European, South Asian, and African cohorts, as well as, the East Asian cohort from the Biobank Japan. Thus, each trait was analyzed in a minimum of four ancestries and a maximum of seven ancestries. 
bioRxiv preprint doi: https://doi.org/10.1101/2021.04.20.440612; this version posted April 20, 2021. The copyright holder for this preprint (which

was not certified by peer review) is the author/funder, who has granted bioRxiv a license to display the preprint in perpetuity. It is made available under aCC-BY-NC-ND 4.0 International license.

\begin{tabular}{|c|c|c|c|c|c|}
\hline Trait Name or Code & Sample Size & Total SNPs & Pruned SNPs & Regions & Citations \\
\hline Basophil count & 62,076 & $5,653,566$ & 410,465 & 23,106 & {$[99$} \\
\hline BMI & 158,284 & $5,653,566$ & 410,465 & 23,085 & {$[98$} \\
\hline CRP & 75,391 & $5,608,701$ & 408,166 & 23,108 & {$[99$} \\
\hline DBP & 136,615 & $5,653,566$ & 410,465 & 23,085 & {$[99$} \\
\hline eGFR & 143,658 & $5,608,701$ & 408,166 & 23,108 & {$[99$} \\
\hline Eosinophil count & 62,076 & $5,653,566$ & 410,465 & 23,106 & {$[99$} \\
\hline HDL & 70,657 & $5,608,701$ & 408,166 & 23,108 & {$[99$} \\
\hline Height & 159,095 & $6,296,332$ & 354,647 & 23,679 & {$[00$} \\
\hline Hematocrit & 108,757 & $5,653,566$ & 410,465 & 23,106 & {$[99$} \\
\hline Hemoglobin & 108,769 & $5,653,566$ & 410,465 & 23,085 & {$[99]$} \\
\hline HbA1c & 75,391 & $5,608,701$ & 408,166 & 23,108 & {$[99$} \\
\hline LDL & 72,866 & $5,608,701$ & 408,166 & 23,108 & {$[99$} \\
\hline Lymphocyte count & 62,076 & $5,653,566$ & 410,465 & 23,106 & {$[99$} \\
\hline MCH & 108,054 & $5,653,566$ & 410,465 & 23,106 & {$[99$} \\
\hline MCHC & 108,738 & $5,653,566$ & 410,465 & 23,106 & {$[99]$} \\
\hline MCV & 108,526 & $5,653,566$ & 410,465 & 23,085 & {$[99]$} \\
\hline Monocyte count & 62,076 & $5,653,566$ & 410,465 & 23,106 & {$[99$} \\
\hline Neutrophil count & 62,076 & $5,653,566$ & 410,465 & 23,106 & {$[99$} \\
\hline PLC & 108,208 & $5,653,566$ & 410,465 & 23,085 & {$[99]$} \\
\hline RBC & 108,794 & $5,653,566$ & 410,465 & 23,085 & {$[99]$} \\
\hline SBP & 136,597 & $5,653,566$ & 410,465 & 23,085 & {$[99]$} \\
\hline Cholesterol & 128,305 & $5,608,701$ & 408,166 & 23,108 & {$[99$} \\
\hline Triglyceride & 105,597 & $5,608,701$ & 410,465 & 23,108 & {$[99$} \\
\hline Urate & 109,029 & $5,608,701$ & 408,166 & 23,108 & {$[99$} \\
\hline WBC & 107,694 & $5,653,566$ & 408,166 & 23,085 & {$[99$} \\
\hline
\end{tabular}

Supplementary Table 4: Number of individuals, total SNPs, pruned SNPs used for gene- $\varepsilon$, and genes and transcription factors (regions) included in the analysis for each trait in Biobank Japan data. Regions were defined using the hg19 list provided in Gusev et al.104. 


\begin{tabular}{|c|c|c|c|c|}
\hline Trait Name or Code & Sample Size & Total SNPs & Pruned SNPs & Regions \\
\hline BMI & 17,127 & $12,139,115$ & 404,401 & 24,216 \\
\hline CRP & 8,349 & $12,274,126$ & 404,572 & 24,206 \\
\hline DBP & 11,380 & $12,148,801$ & 405,188 & 24,218 \\
\hline eGFR & 8,261 & $12,128,273$ & 403,371 & 24,207 \\
\hline Hemoglobin A1c & 17,127 & $12,139,115$ & 404,401 & 24,215 \\
\hline HDL & 10,085 & $12,114,827$ & 404,089 & 24,201 \\
\hline Height & 17,280 & $12,139,907$ & 404,522 & 24,201 \\
\hline LDL & 9,720 & $12,107,344$ & 403,740 & 24,218 \\
\hline MCHC & 3,750 & $12,132,232$ & 405,558 & 24,217 \\
\hline PLC & 8,850 & $12,131,935$ & 404,497 & 24,193 \\
\hline SBP & 11,380 & $12,148,801$ & 405,188 & 24,218 \\
\hline Cholesterol & 10,137 & $12,110,337$ & 403,674 & 24,222 \\
\hline Triglyceride & 9,980 & $12,110,879$ & 403,455 & 24,206 \\
\hline WBC & 8,802 & $12,126,732$ & 404,579 & 24,219 \\
\hline
\end{tabular}

Supplementary Table 5: Number of individuals, total SNPs, pruned SNPs used for gene- $\varepsilon$, and genes and transcription factors (regions) included in the analysis for each trait in the African-American cohort of the PAGE data.

\begin{tabular}{|c|c|c|c|c|}
\hline Trait Name or Code & Sample Size & Total SNPs & Pruned SNPs & Region Count \\
\hline BMI & 21,955 & $8,812,436$ & 432,762 & 24,138 \\
\hline CRP & 15,912 & $8,576,621$ & 397,941 & 24,118 \\
\hline DBP & 21,549 & $8,784,112$ & 430,360 & 24,126 \\
\hline Estimated glomerular filtration rate & 18,548 & $8,702,426$ & 422,598 & 24,123 \\
\hline HbA1c & 21,955 & $8,812,436$ & 432,762 & 24,138 \\
\hline HDL & 17,751 & $8,583,603$ & 412,771 & 24,122 \\
\hline Height & 22,187 & $8,822,606$ & 433,604 & 24,132 \\
\hline LDL & 17,373 & $8,588,800$ & 413,074 & 24,116 \\
\hline MCHC & 15,522 & $8,763,739$ & 427,208 & 24,132 \\
\hline PLC & 18,949 & $8,612,804$ & 415,201 & 24,115 \\
\hline SBP & 21,549 & $8,784,112$ & 430,360 & 24,126 \\
\hline Cholesterol & 17,802 & $8,586,887$ & 412,830 & 24,115 \\
\hline Triglyceride & 17,856 & $8,594,121$ & 413,546 & 24,104 \\
\hline WBC & 18,206 & $8,603,503$ & 414,462 & 24,123 \\
\hline
\end{tabular}

Supplementary Table 6: Number of individuals, total SNPs, pruned SNPs used for gene- $\varepsilon$, and genes and transcription factors (regions) included in the analysis for each trait in the Hispanic and Latin American cohort of the PAGE data. 


\begin{tabular}{|c|c|c|c|c|}
\hline Trait Name or Code & Sample Size & Total SNPs & Pruned SNPs & Regions \\
\hline BMI & 645 & $8,374,976$ & 421,826 & 24,124 \\
\hline CRP & 574 & $8,504,922$ & 417,287 & 24,136 \\
\hline DBP & 636 & $8,376,521$ & 421,528 & 24,136 \\
\hline eGFR & 602 & $8,336,044$ & 417,540 & 24,132 \\
\hline Hemoglobin A1c & 645 & $8,374,976$ & 421,826 & 24,124 \\
\hline HDL & 604 & $8,315,912$ & 415,939 & 24,121 \\
\hline Height & 645 & $8,375,624$ & 421,750 & 24,117 \\
\hline LDL & 591 & $8,360,719$ & 419,544 & 24,123 \\
\hline MCHC & 20 & $3,970,246$ & 62,339 & 17,381 \\
\hline PLC & 603 & $8,294,302$ & 414,530 & 24,133 \\
\hline Systolic blood pressure & 636 & $8,376,521$ & 421,528 & 24,136 \\
\hline Cholesterol & 604 & $8,586,887$ & 415,939 & 24,121 \\
\hline WBC & 602 & $8,289,567$ & 414,462 & 24,133 \\
\hline
\end{tabular}

Supplementary Table 7: Number of individuals, total SNPs, pruned SNPs used for gene- $\varepsilon$, and genes and transcription factors (regions) included in the analysis for each trait in the AIAN cohort of the PAGE data.

\begin{tabular}{|c|c|c|c|c|}
\hline Trait Name or Code & Sample Size & Total SNPs & Pruned SNPs & Regions \\
\hline BMI & 3,936 & $6,664,738$ & 415,221 & 23,885 \\
\hline CRP & 1,777 & $6,966,169$ & 428,517 & 23,834 \\
\hline Hemoglobin A1c & 3,936 & $6,664,738$ & 415,221 & 23,885 \\
\hline HDL & 1,912 & $6,656,996$ & 416,255 & 23,894 \\
\hline Height & 3,938 & $6,660,920$ & 415,172 & 23,878 \\
\hline LDL & 1,900 & $6,662,802$ & 416,810 & 23,895 \\
\hline Cholesterol & 1,915 & $6,660,807$ & 416,425 & 23,899 \\
\hline Triglycerides & 1,915 & $6,660,807$ & 416,425 & 23,899 \\
\hline
\end{tabular}

Supplementary Table 8: Number of individuals, total SNPs, pruned SNPs used for gene- $\varepsilon$, and genes and transcription factors (regions) included in the analysis for each trait in the Native Hawaiian (Native Hawaiian) cohort of the PAGE data. 


\begin{tabular}{|c|c|c|c|c|}
\hline Trait Name or Code & Sample Size & Total SNPs & Pruned SNPs & Regions \\
\hline BMI & 4,647 & $15,362,633$ & 433,356 & 24,085 \\
\hline CRP & 1,811 & $14,374,461$ & 428,656 & 24,116 \\
\hline DBP & 1,086 & $12,470,507$ & 416,273 & 24,112 \\
\hline eGFR & 150 & $8,314,417$ & 337,167 & 24,017 \\
\hline HbA1c & 4,647 & $15,362,633$ & 433,356 & 24,085 \\
\hline HDL & 2,378 & $13,413,244$ & 428,598 & 24,072 \\
\hline Height & 4,679 & $15,366,710$ & 433,005 & 24,103 \\
\hline LDL & 2,316 & $13,327,313$ & 428,741 & 24,075 \\
\hline MCHC & 128 & $8,089,136$ & 315,583 & 23,946 \\
\hline PLC & 541 & $10,528,072$ & 421,929 & 24,098 \\
\hline SBP & 1,086 & $12,470,507$ & 416,273 & 24,112 \\
\hline Cholesterol & 2,387 & $13,436,190$ & 428,656 & 24,078 \\
\hline Triglyceride & 2,381 & $13,423,953$ & 429,246 & 24,073 \\
\hline WBC & 543 & $10,570,051$ & 421,776 & 24,095 \\
\hline
\end{tabular}

Supplementary Table 9: Number of individuals, total SNPs, pruned SNPs used for gene- $\varepsilon$, and genes and transcription factors (regions) included in the analysis for each trait in the Asian cohort of the PAGE data. 


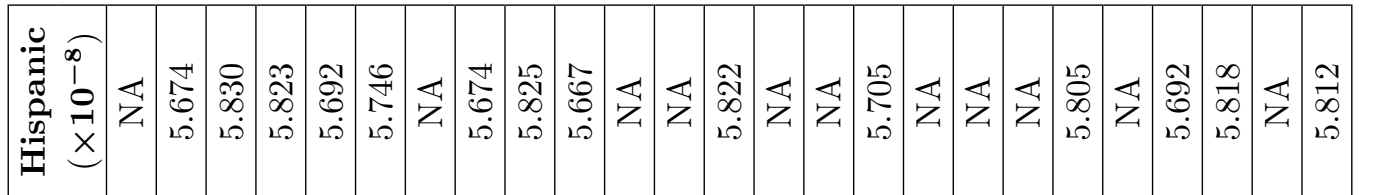

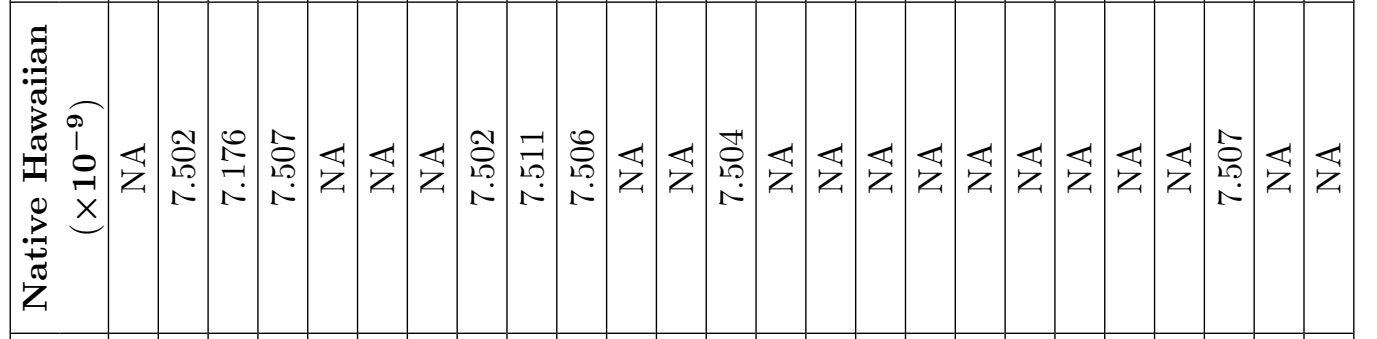

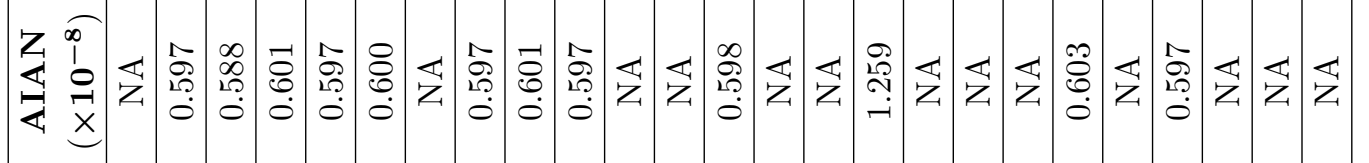

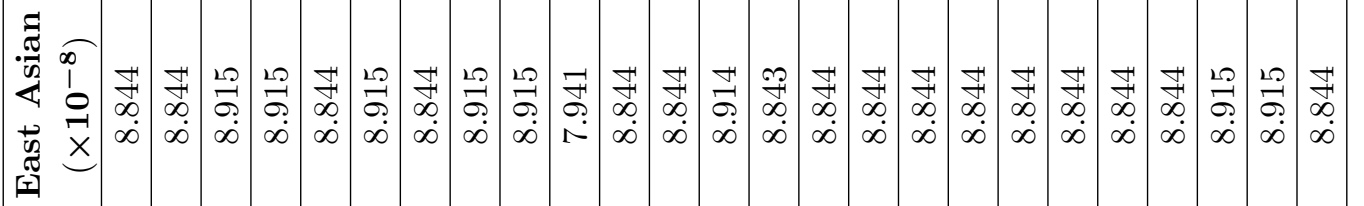

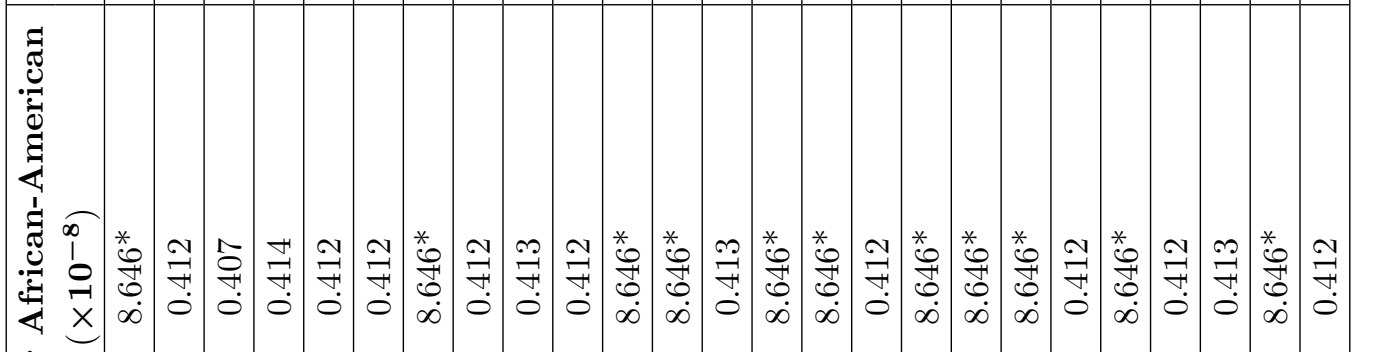

ó

(ู)

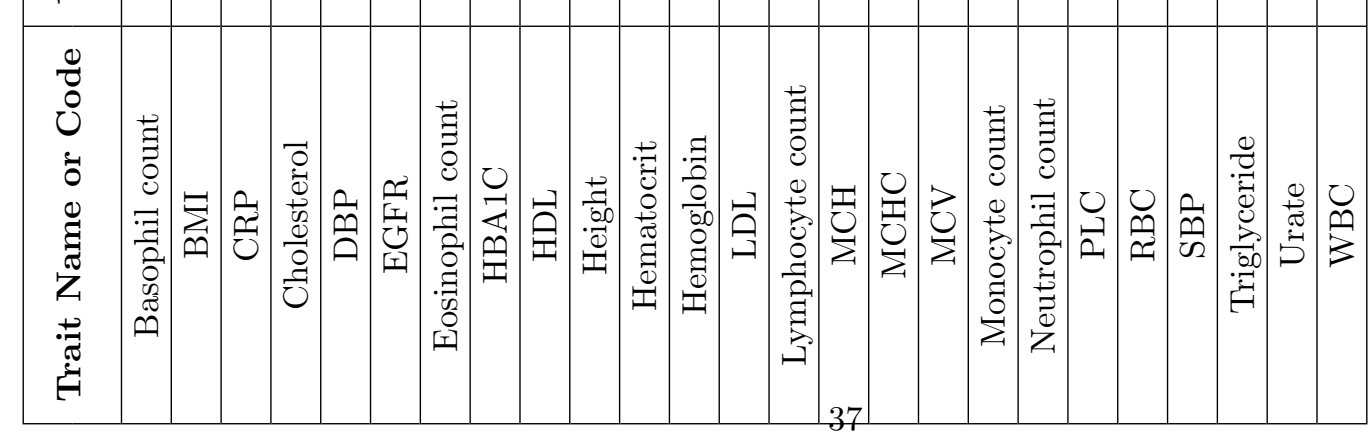

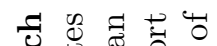

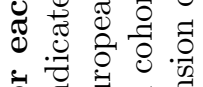

苟.

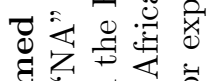

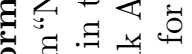

कृ है

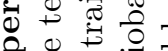

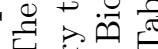

के

范 0

.

需

0

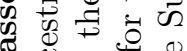

D.

임 矛过

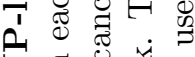

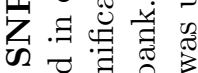

क .0

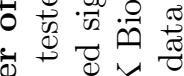

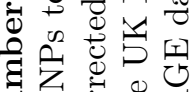

望导导

च

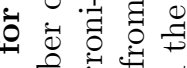

ठ艹

경

of

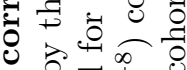

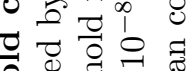

过岛 $\times$

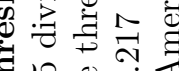

İ 20 \&

$0 \quad \dot{F} \vee \frac{\pi}{0}$

శิ

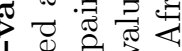

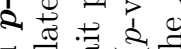

규

సี

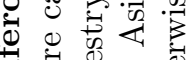

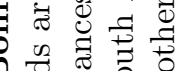

党 $\begin{aligned} & 0 \\ & 0\end{aligned}$

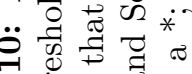

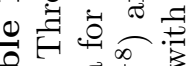

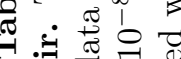

$\lambda$ 政

을 웅

需

范

$\sum_{0} V$ D

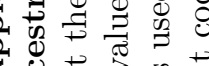

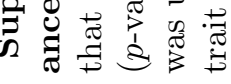




\begin{tabular}{|c|c|c|c|c|}
\hline $\begin{array}{c}\text { Trait Name or } \\
\text { Code }\end{array}$ & $\begin{array}{c}\text { Median Effect } \\
\text { Size Correlation }\end{array}$ & $\begin{array}{c}\text { Effect Sizes } \\
>0.1 \text { (Mean) }\end{array}$ & $\begin{array}{c}\text { Median PIP } \\
\text { Correlation } \\
\end{array}$ & $\begin{array}{c}\text { PIPs }>0.01 \\
(\text { Mean })\end{array}$ \\
\hline BMI & 0.0143 & 0 & 0.073 & 67.3 \\
\hline Basophil & $3.69 \times 10^{-6}$ & 0 & 0.002 & 211.8 \\
\hline $\mathrm{CRP}$ & 0.096 & 0.7 & 0.159 & 142.6 \\
\hline Cholesterol & 0.966 & 1 & 0.232 & 53.1 \\
\hline DBP & 0.001 & 0.6 & 0.012 & 173.1 \\
\hline EGFR & $4.71 \times 10^{-6}$ & 0 & 0.005 & 149 \\
\hline Eosinophil & 0.0179 & 0.2 & 0.042 & 325.8 \\
\hline HBA1C & 0.012 & 0.2 & 0.025 & 26.8 \\
\hline HDL & 0.325 & 0.1 & 0.219 & 57.6 \\
\hline Height & $9.20 \times 10^{-5}$ & 1.6 & 0.010 & 187.7 \\
\hline Hematocrit & $-4.16 \times 10^{-6}$ & 0.5 & 0.015 & 40.3 \\
\hline Hemoglobin & $-1.13 \times 10^{-5}$ & 0.6 & 0.014 & 45.8 \\
\hline LDL & 0.949 & 1 & 0.331 & 60.9 \\
\hline Lymphocyte & $-1.01 \times 10^{-6}$ & 1.5 & 0.001 & 1368.5 \\
\hline $\mathrm{MCH}$ & $7.098 \times 10^{-6}$ & 0.6 & 0.007 & 60.9 \\
\hline $\mathrm{MCHC}$ & $-2.00 \times 10^{-6}$ & 0.9 & 0.01 & 58.7 \\
\hline $\mathrm{MCV}$ & $1.71 \times 10^{-5}$ & 0.6 & 0.01 & 70 \\
\hline Monocyte & 0.002 & 0.1 & 0.017 & 349.4 \\
\hline Neutrophil & 0.038 & 0.1 & 0.071 & 66.5 \\
\hline PLC & 0.609 & 0.9 & 0.154 & 112.5 \\
\hline $\mathrm{RBC}$ & $9.650 \times 10^{-5}$ & 0.9 & 0.028 & 49.7 \\
\hline SBP & 0.001 & 0.6 & 0.019 & 166.8 \\
\hline Triglyceride & 0.348 & 0.0 & 0.247 & 164.7 \\
\hline Urate & 0.252 & 0.5 & 0.24 & 39.9 \\
\hline WBC & 0.0002 & 0.1 & 0.01 & 347.8 \\
\hline
\end{tabular}

Supplementary Table 11: Replication of effect sizes and posterior inclusion probabilities (PIPs) among ten independent subsamples of the UK Biobank European ancestry cohort using $\mathrm{SuSiE}^{46}$ for fine-mapping. The sample size of the ten independent, non-overlapping subsamples of the UK Biobank European ancestry cohort was set to 10,000. For the 1,895,051 SNPs that were analyzed in every European ancestry cohort subsample (Supplementary Table 1) and the effect sizes and PIPs (columns 2 and 4, respectively) generated using the $\mathrm{SuSiE}$ method ${ }^{46}$, we calculated the median correlation coefficient between all possible pairwise comparisons (10 choose 2) of the European ancestry cohort subsamples. Column 3 reports the mean number of SNPs with effect sizes greater than 0.1 across all ten European ancestry cohort subsamples for each trait. Column 5 reports the mean number of SNPs with a posterior inclusion probability greater than 0.01 across the ten European ancestry cohort subsamples for each trait. 
bioRxiv preprint doi: https://doi.org/10.1101/2021.04.20.440612; this version posted April 20, 2021. The copyright holder for this preprint (which was not certified by peer review) is the author/funder, who has granted bioRxiv a license to display the preprint in perpetuity. It is made

คิ ava lable under aCC-BY-NC-ND 4.0 International license.

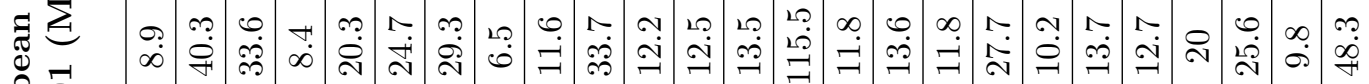

$\stackrel{0}{0}$

氠 1

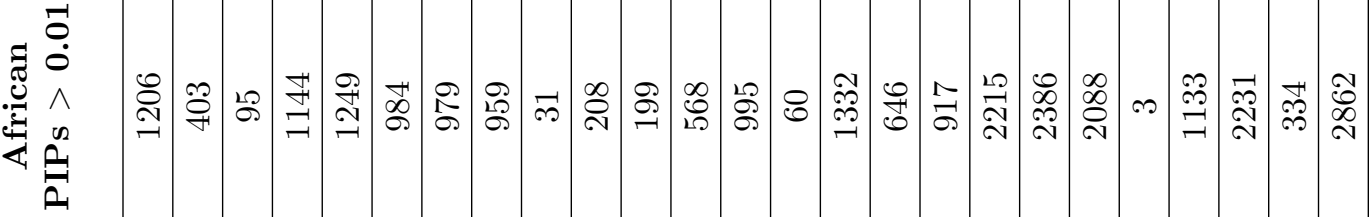

ㄴ.

สี $\frac{\pi}{0}$

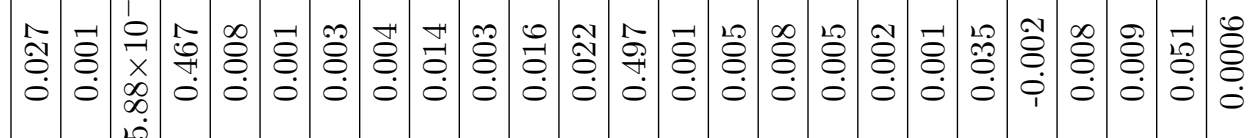

تृ

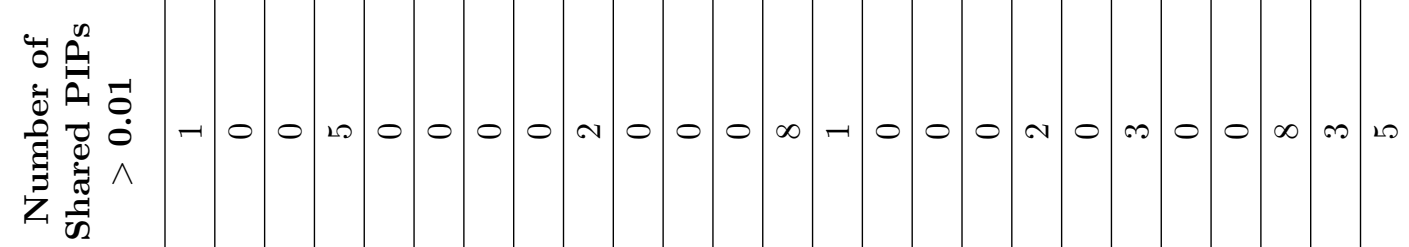

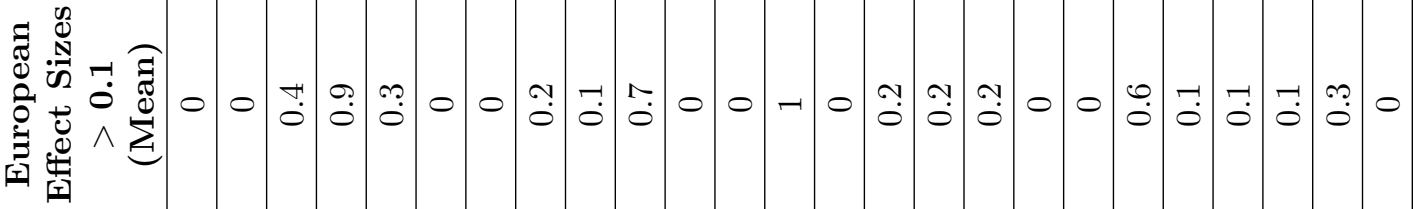

过

되

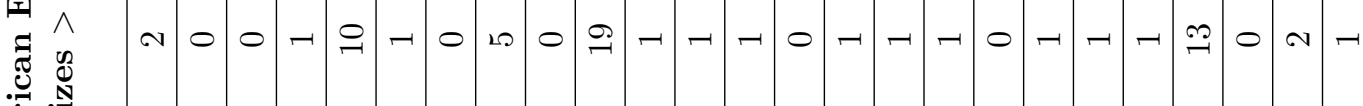

in

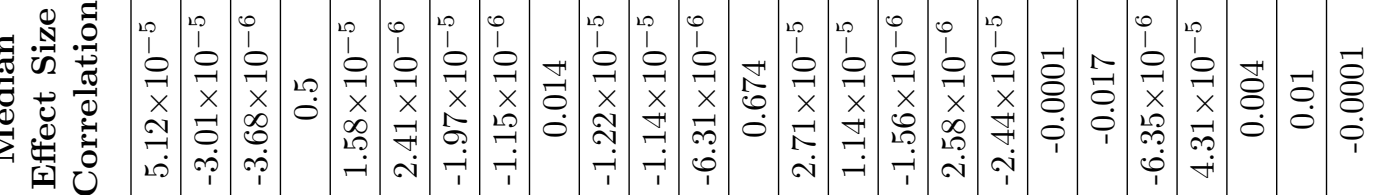

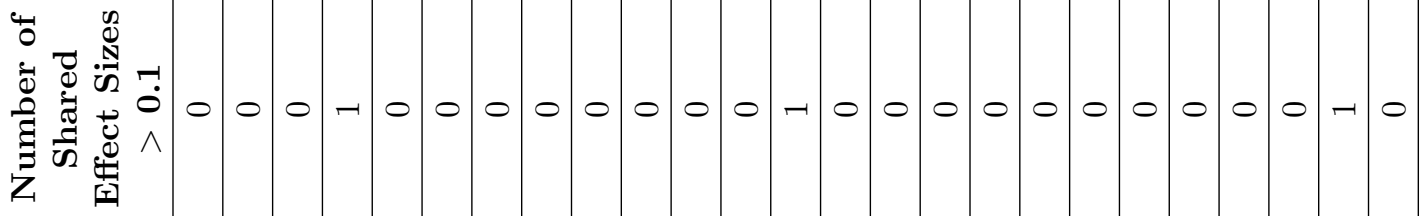

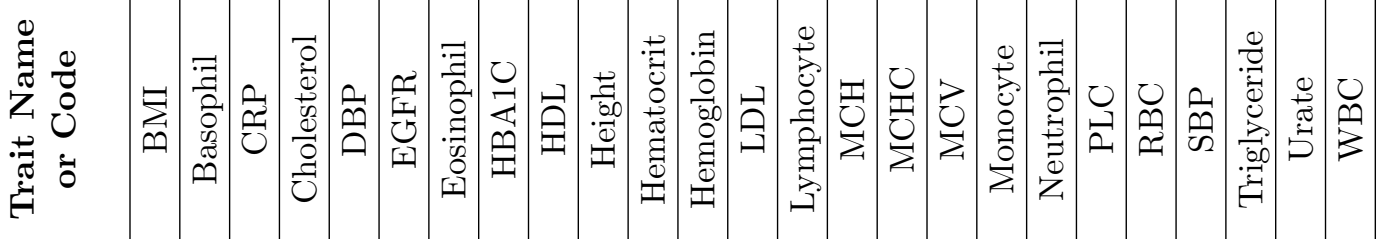

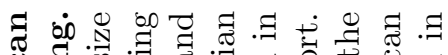

.

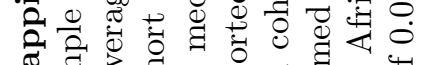

శ్ శี

है

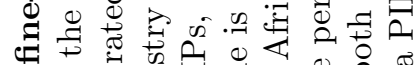

世

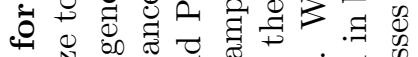

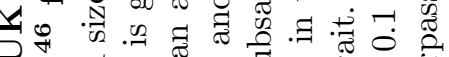

I $\exists$ चี

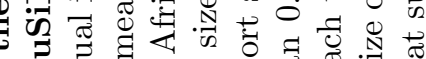

क ज

20 $00 \%$ \&

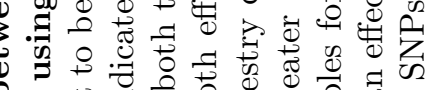

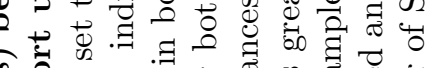

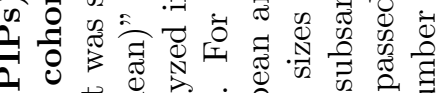

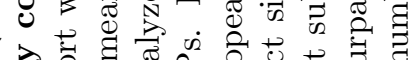

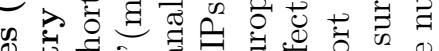

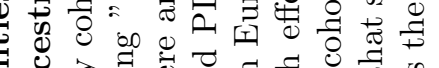

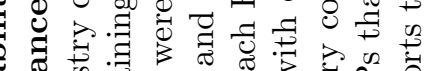

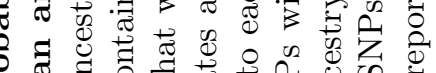



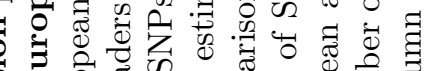

国

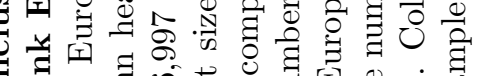

ส

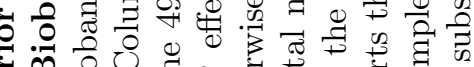

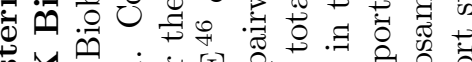

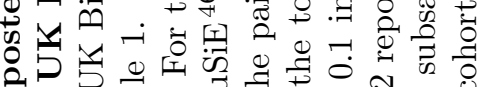

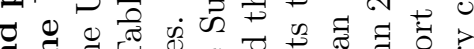

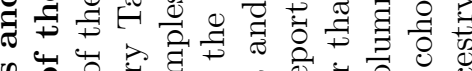

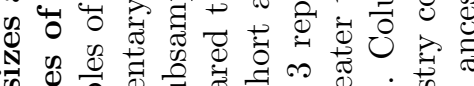

क है द्व

ల छ

过

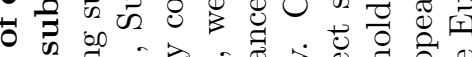

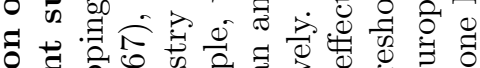

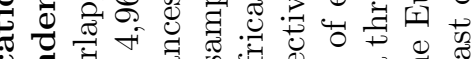

㐘

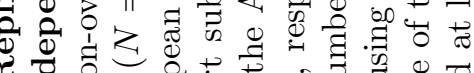

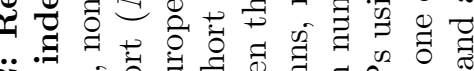

N

๑

क

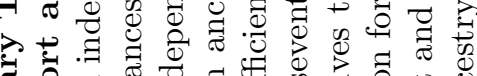

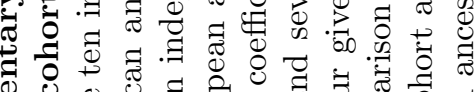

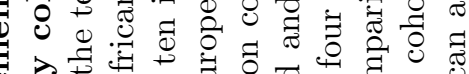

0

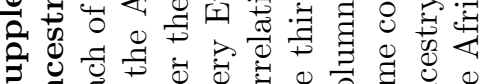

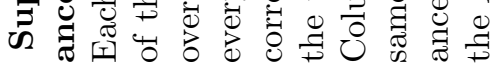


bioRxiv preprint doi: https://doi.org/10.1101/2021.04.20.440612; this version posted April 20, 2021. The copyright holder for this preprint (which was not certified by peer review) is the author/funder, who has grahted bioRxiv a license to display the preprint in perpetuity. It is made

คి ava lable under aCC-BY-NC-ND 4.0 International license.

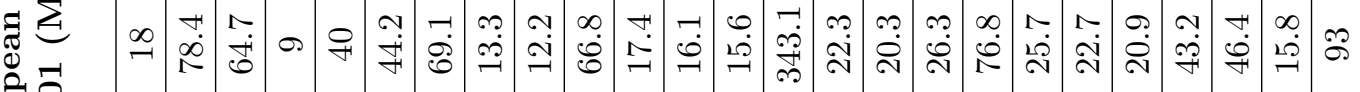

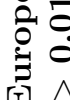

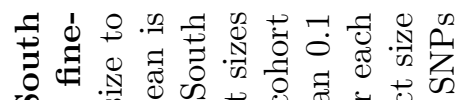

ڤ

สี ฮิ

$\cdot \frac{\pi}{\pi} \dot{0}$

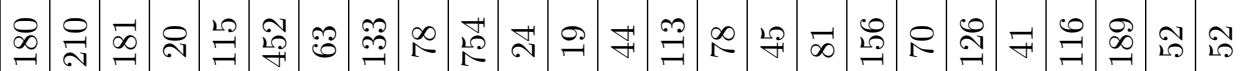

in

สี

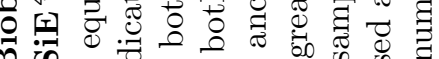

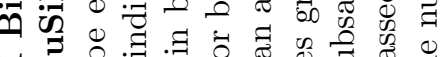

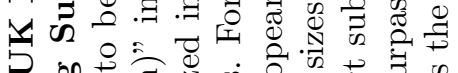

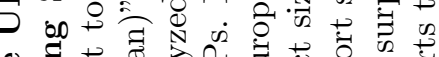

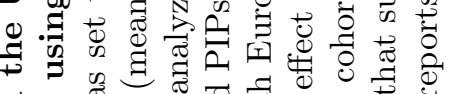

A

A

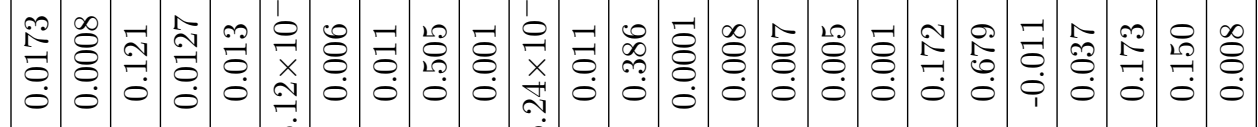

可

$\sum_{i}^{0} 0$

$+\pi=\pi \quad z=0$

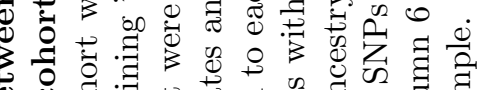

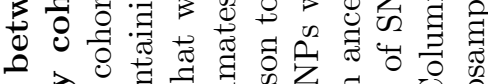

क

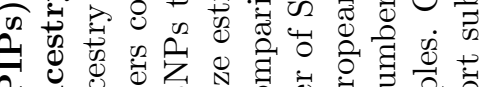

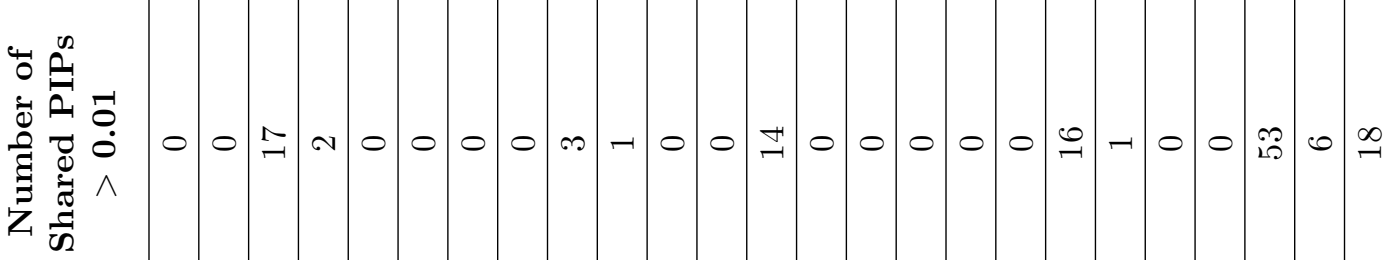

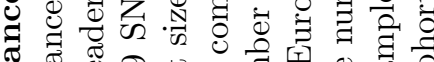

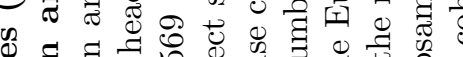

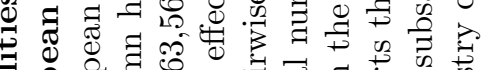

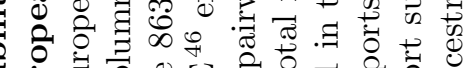

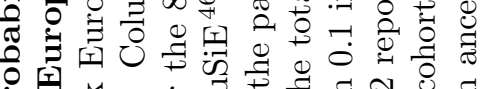

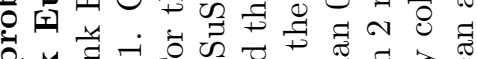

कै

สี สิ응

品

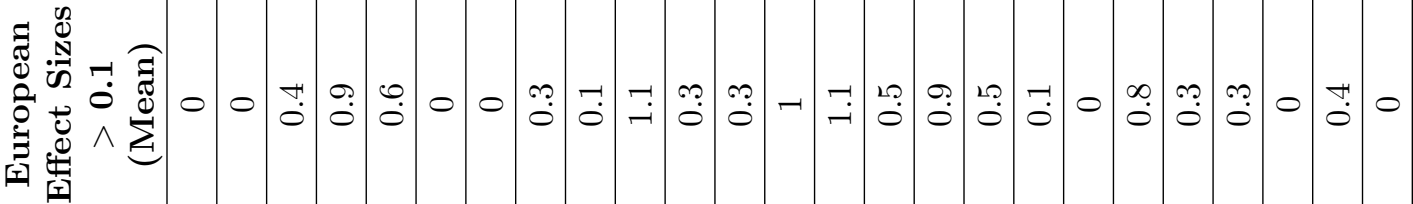

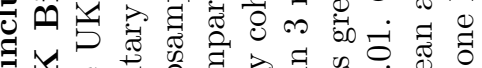

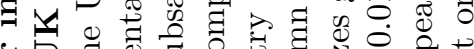

oิ

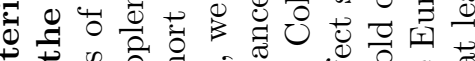

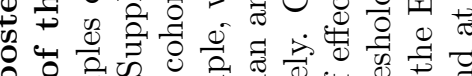

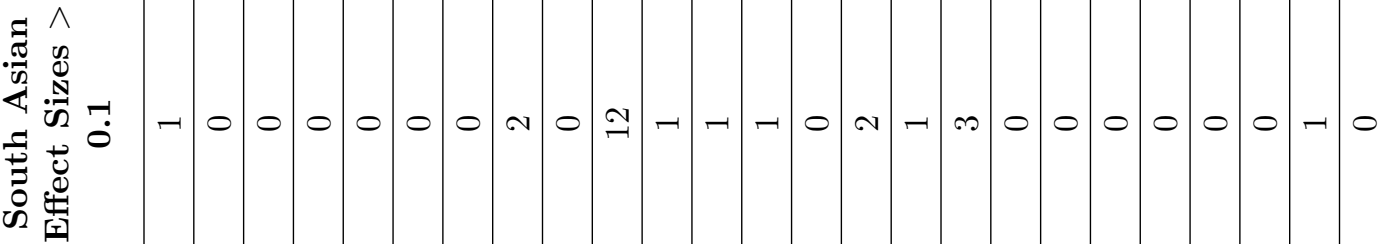

n

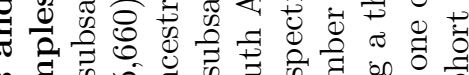

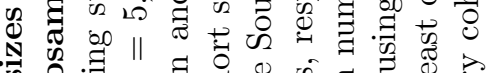

क

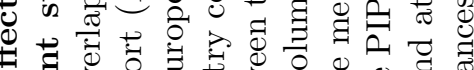

बै 0

过

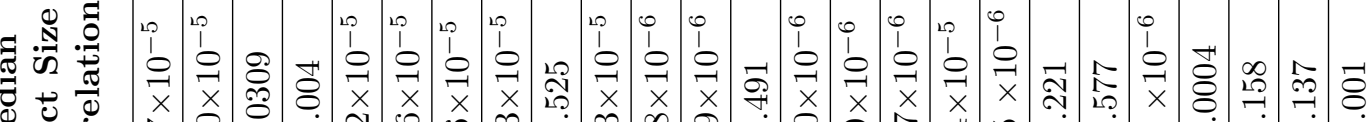

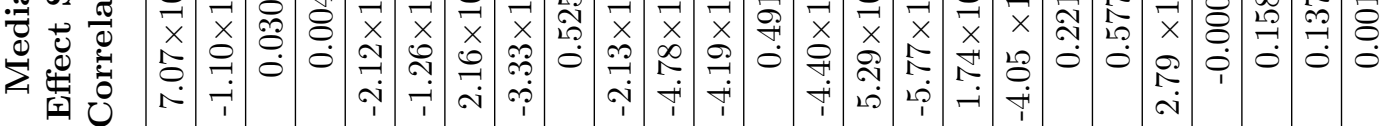

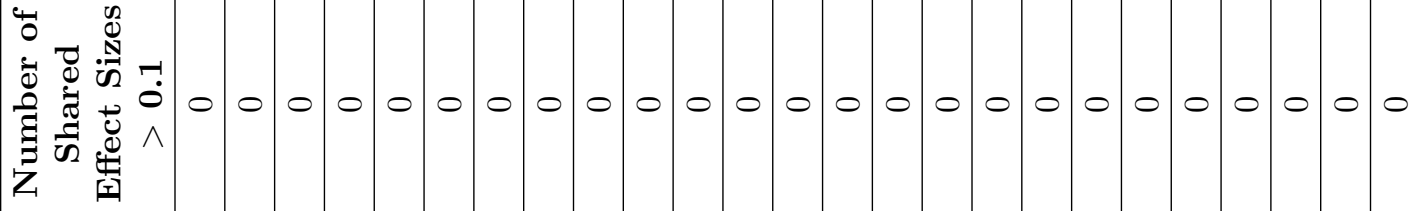

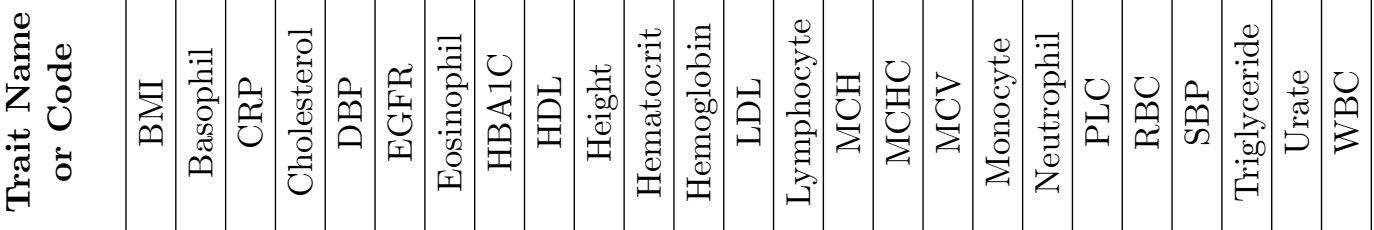

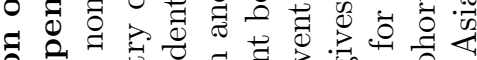

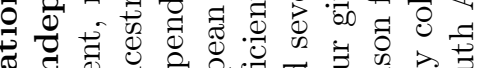

记

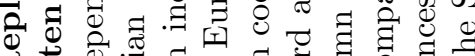

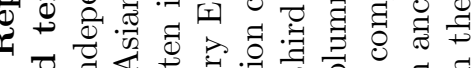

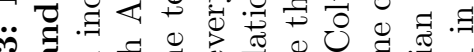

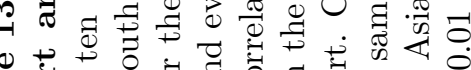

0.

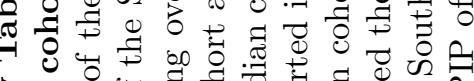

ते

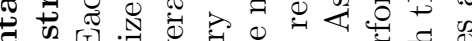

की

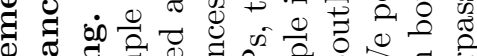

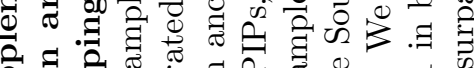

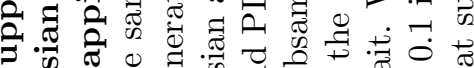

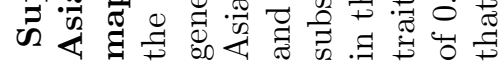




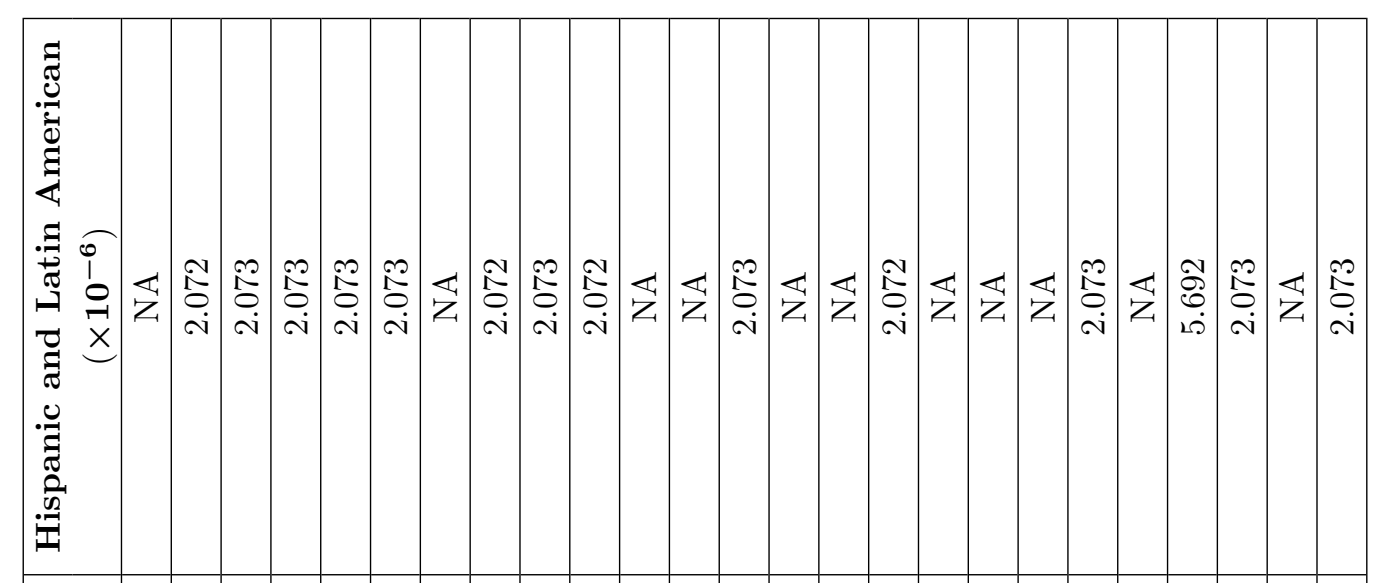

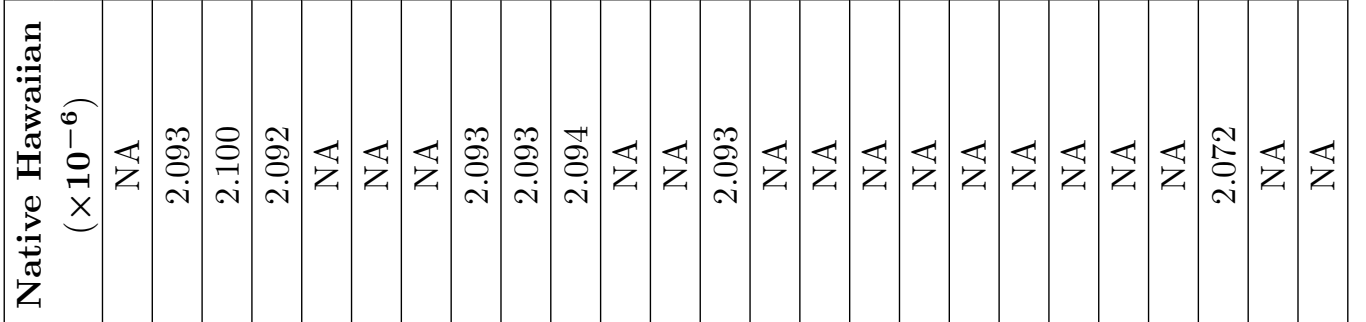

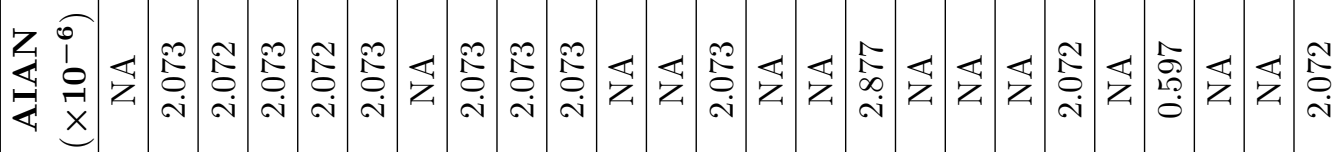

晃

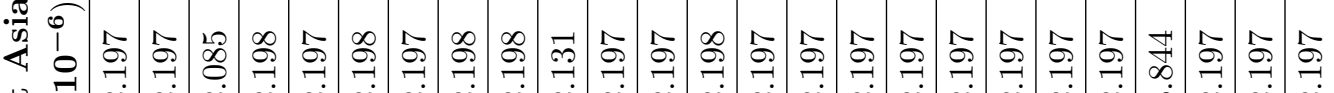

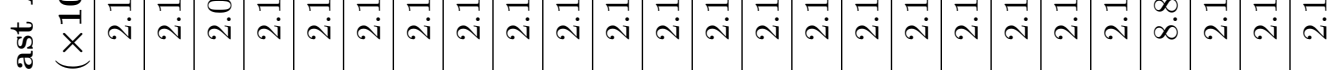
疍

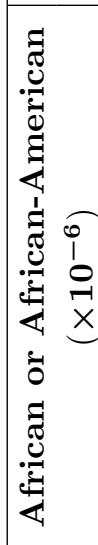

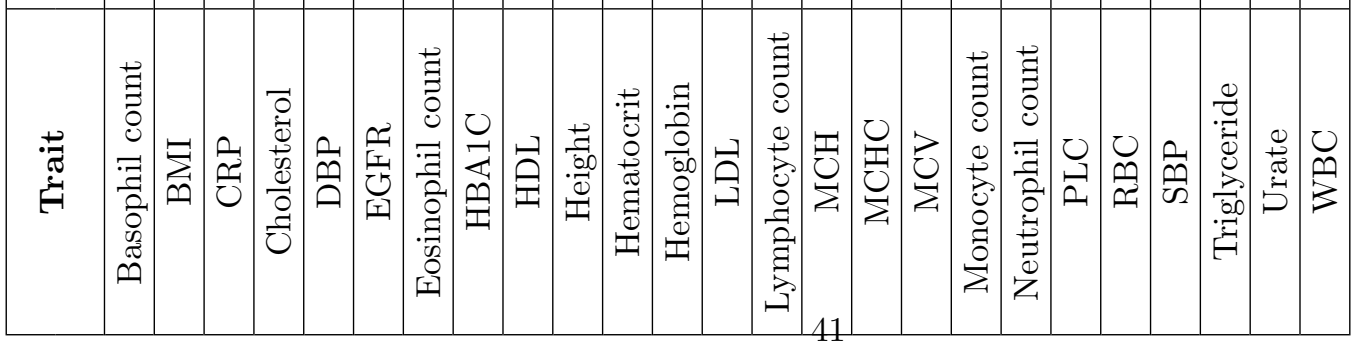

궁 प्र ठ․ 过节四

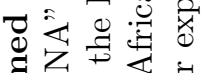
. 룽 क

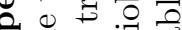
$\rightarrow$ 䨔

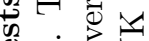
.ं $ठ 5$ 菅

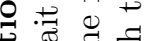

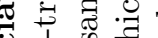
30 至

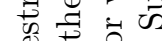

శ $\mathbb{C}_{0}$ is व 궁 㐘出 बं

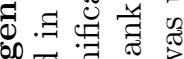
-

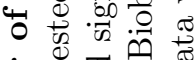

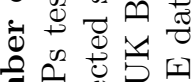

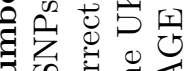

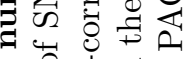
ᄒ․ ठदे औ्ञ ज

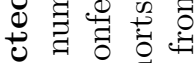
ช.

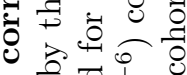
둥후을 . $0: \exists$ 웡

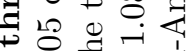
○芦 $\vee$ ส

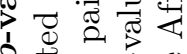

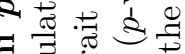

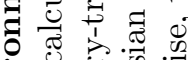

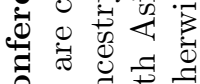
वृ ต 궁

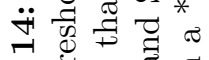
웥 东䒕 ส 의웅 ส. 궝 踏 O V D 式恶焉 d

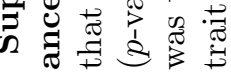


bioRxiv preprint doi: https://doi.org/10.1101/2021.04.20.440612; this version posted April 20, 2021. The copyright holder for this preprint (which

was not certified by peer review) is the author/funder, who has granted bioRxiv a license to display the preprint in perpetuity. It is made available under aCC-BY-NC-ND 4.0 International license.

\begin{tabular}{|c|c|c|c|c|}
\hline Gene & African or African-American & European & East Asian & Hispanic \\
\hline ANGPTL4 & 110 & 111 & NA & 111 \\
\hline$A P O A 1$ & 112 & 112 & NA & 112 \\
\hline $\mathrm{APOA}_{4}$ & $\mathrm{NA}$ & 113 & $\mathrm{NA}$ & $\mathrm{NA}$ \\
\hline$A P O A 5$ & 114 & \begin{tabular}{|l|l|}
114 & 117 \\
\end{tabular} & 118 & 114 \\
\hline$A P O B$ & 119 & 119 & NA & 119 \\
\hline$A P O C 1$ & 112 & 113 & $\mathrm{NA}$ & 112 \\
\hline$A P O C 2$ & 114 & 114 & NA & 114 \\
\hline АРОСЗ & 112 & \begin{tabular}{|l|l|l|}
112 & 113 \\
\end{tabular} & NA & 112 \\
\hline $\mathrm{APOC}_{4}$ & 113 & 113 & NA & 113 \\
\hline$A P O E$ & 113 & $113 \mid 114$ & NA & 113 \\
\hline CETP & 112 & 113 & NA & 112 \\
\hline$L M F 1$ & $\mathrm{NA}$ & 113 & $\mathrm{NA}$ & $\mathrm{NA}$ \\
\hline$L P L$ & 112 & 113 & 118 & 112 \\
\hline PCSK6 & 110 & 113 & $\mathrm{NA}$ & 110 \\
\hline PCSKY & 114 & 114 & 118 & 114 \\
\hline PLTP & \begin{tabular}{|l|l|l|l|}
$111 \mid 112$ & 114 & 119 \\
\end{tabular} & \begin{tabular}{|l|l|l|l|}
111 & 113 & 119 & 121 \\
\end{tabular} & $\mathrm{NA}$ & \begin{tabular}{|l|l|l|l|}
111 & 112 & 114 & 119 \\
\end{tabular} \\
\hline
\end{tabular}

Supplementary Table 15: Genes shown in Figure 3 as associated with triglyceride levels are supported by publications in the GWAS Catalog. Each of the genes listed is present in the significantly mutated subnetworks identified using Hierarchical HotNet $\underline{61}$ as enriched for associations with triglyceride levels in the European, East Asian, or Native Hawaiian ancestry cohorts. We mapped SNP-level associations from the GWAS Catalog to the 29 genes present in the significantly mutated subnetworks shown in Figure 3 (using the gene list provided by Gusev et al. ${ }^{104}$ ) to generate the results for the 16 genes shown here. 


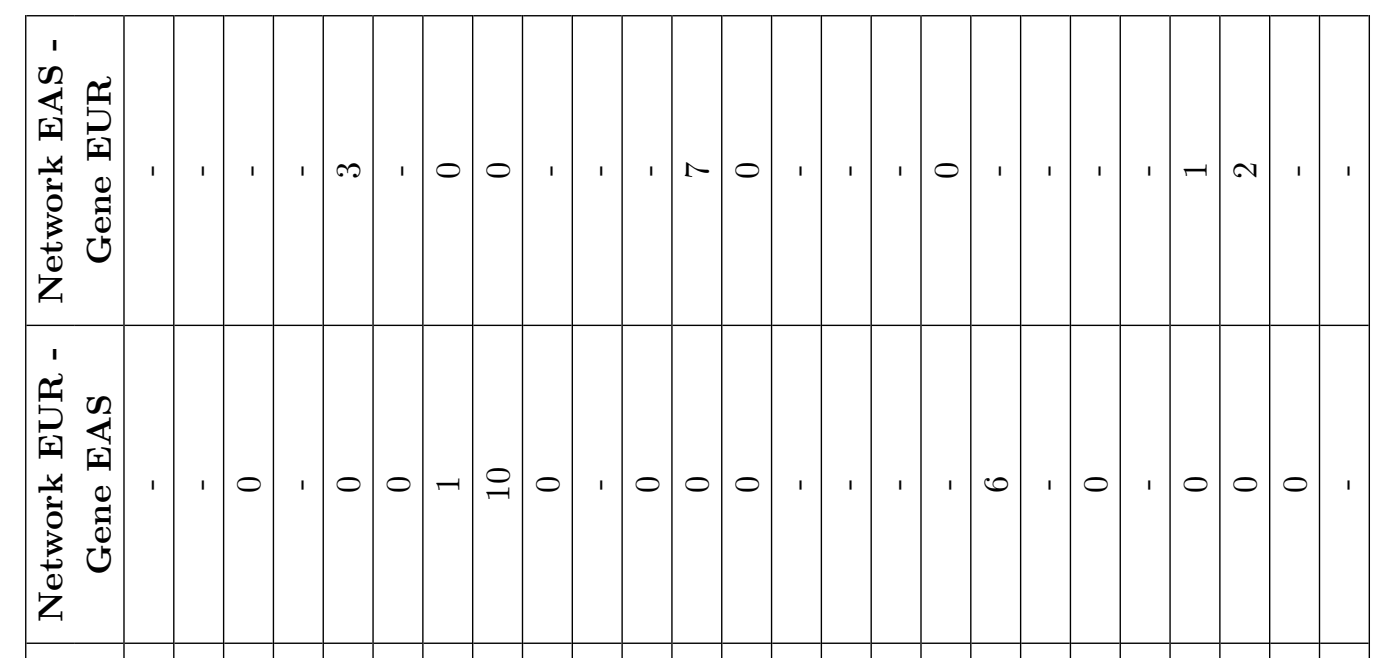

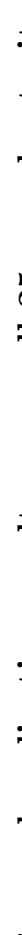

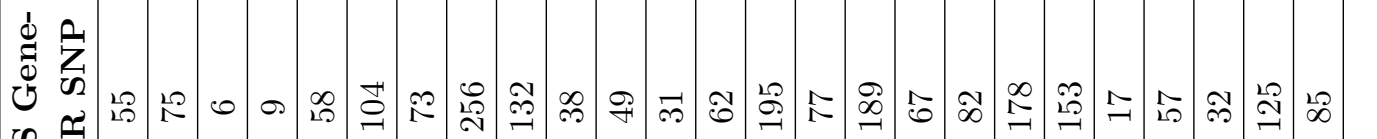
近罢

\begin{tabular}{|c|c|c|c|c|c|c|c|c|c|c|c|c|c|c|c|c|c|c|c|c|c|c|c|c|c|c|}
\hline 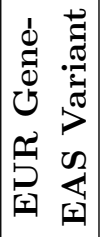 & $\neg$ & $\infty$ & H & $N$ & $\stackrel{m}{1}$ & $\begin{array}{l}2 \\
\end{array}$ & \begin{tabular}{l|l}
0 & 1
\end{tabular} & N & $\ddot{\infty}$ & 그 & $\infty$ & 二 & $\stackrel{ }{\circ}$ & $\neg$ & $\stackrel{\curvearrowright}{\curvearrowright}$ & $\stackrel{\sim}{\sim}$ & 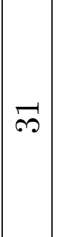 & $\stackrel{120}{-1}$ & $\stackrel{0}{-1}$ & $\stackrel{N}{N}$ & $\infty$ & $\infty$ & $\stackrel{\Omega}{-1}$ & $\stackrel{N}{N}$ & $\stackrel{\infty}{-1}$ & 20 \\
\hline
\end{tabular}

\begin{tabular}{|c|c|c|c|c|c|c|c|c|c|c|c|c|c|c|c|c|c|c|c|c|c|c|c|c|c|}
\hline 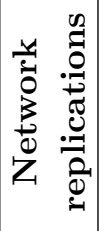 & 1 & 1 & 1 & 1 & 0 & 0 & $\sigma$ & 0 & 1 & 1 & 1 & $\theta$ & -1 & 1 & 1 & 1 & 1 & 1 & 1 & 1 & 1 & $N$ & $\exists$ & 1 & 1 \\
\hline
\end{tabular}

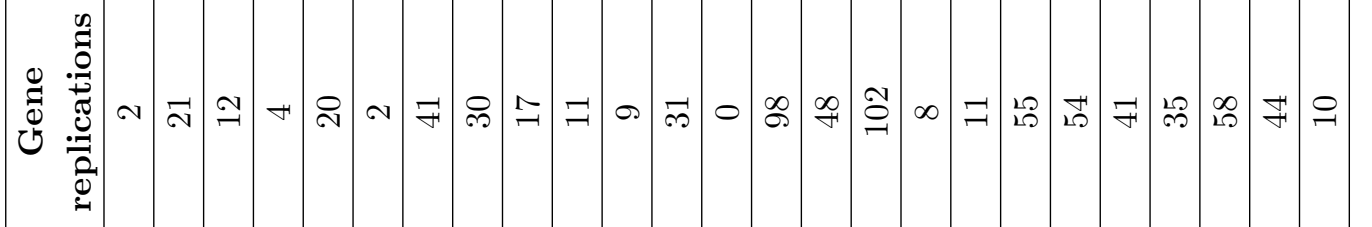

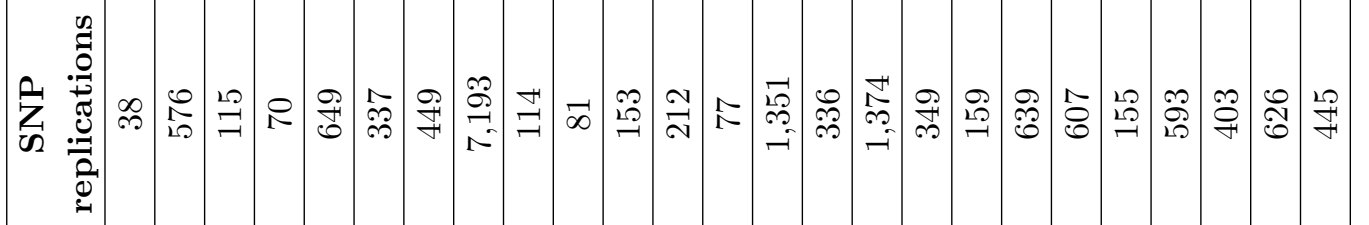

\begin{tabular}{|c|c|c|c|c|c|c|c|c|c|c|c|c|c|c|c|c|c|c|c|c|c|c|c|c|}
\hline 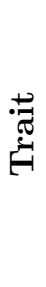 & 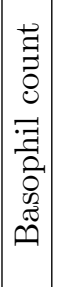 & $\sum_{m}$ & $\frac{\hat{2}}{0}$ & $\overrightarrow{\hat{n}}$ & 至 & 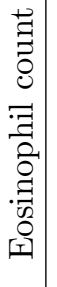 & $\vec{\Theta}$ & 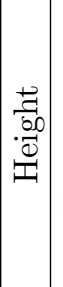 & 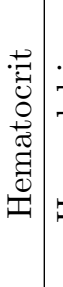 & 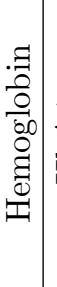 & 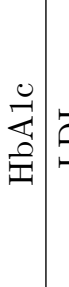 & $\overrightarrow{\mathrm{A}}$ & 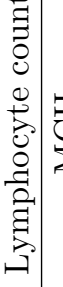 & $\begin{array}{l}\vec{J} \\
己 \\
\end{array}$ & 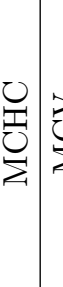 & 它 & 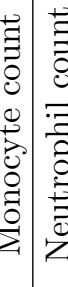 & 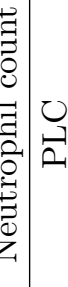 & 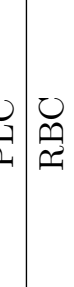 & $\vec{n}$ & 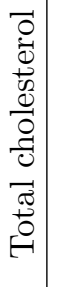 & 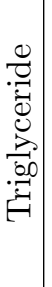 & 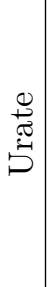 & $\begin{array}{c}0 \\
n \\
3 \\
3\end{array}$ \\
\hline
\end{tabular}

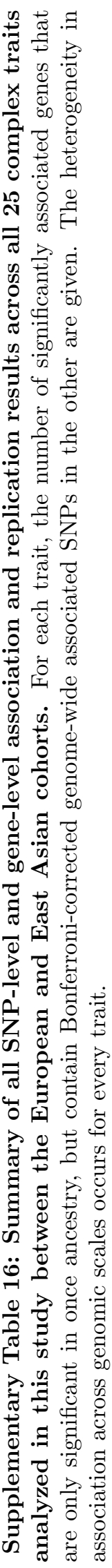


bioRxiv preprint doi: https://doi.org/10.1101/2021.04.20.440612; this version posted April 20, 2021. The copyright holder for this preprint (which

was not certified by peer review) is the author/funder, who has granted bioRxiv a license to display the preprint in perpetuity. It is made available under aCC-BY-NC-ND 4.0 International license.

\begin{tabular}{|c|c|c|c|c|c|c|}
\hline Gene & African-American & European & South Asian & East Asian & AIAN & $\begin{array}{c}\text { Hispanic and } \\
\text { Latin American }\end{array}$ \\
\hline RDH13 & $4.14 \times 10^{-10}$ & $9.95 \times 10^{-1}$ & $8.80 \times 10^{-2}$ & $1.76 \times 10^{-6}$ & 1 & $7.88 \times 10^{-1}$ \\
\hline AGPAT5 & 1 & $1.30 \times 10^{-6}$ & $7.83 \times 10^{-1}$ & $5.00 \times 10^{-1}$ & $7.33 \times 10^{-8}$ & 1 \\
\hline GP6 & $7.20 \times 10^{-10}$ & $9.93 \times 10^{-1}$ & $1.47 \times 10^{-1}$ & $9.07 \times 10^{-7}$ & 1 & $6.92 \times 10^{-1}$ \\
\hline ALDH2 & 1 & $1.00 \times 10^{-20}$ & $1.13 \times 10^{-2}$ & $1.00 \times 10^{-20}$ & 1 & 1 \\
\hline RAB8A & $9.57 \times 10^{-1}$ & $1.00 \times 10^{-20}$ & 1 & $5.76 \times 10^{-6}$ & 1 & $9.97 \times 10^{-1}$ \\
\hline CUX2 & 1 & $5.13 \times 10^{-7}$ & $1.16 \times 10^{-1}$ & $3.44 \times 10^{-11}$ & 1 & 1 \\
\hline ACAD10 & 1 & $1.47 \times 10^{-10}$ & $1.10 \times 10^{-2}$ & $2.00 \times 10^{-10}$ & 1 & 1 \\
\hline
\end{tabular}

Supplementary Table 17: Gene-level association $p$-values for seven genes associated with platelet count in at last two ancestry cohorts. Of the 65 genes that were associated with platelet count in at least two ancestry cohorts, these seven contained previously identified SNP-level associations in studies submitted to the GWAS Catalog. Previous associations in the GWAS Catalog are discussed in the Supplementary Note. Ancestry-specific Bonferroni corrected significance thresholds for gene-level association analysis of platelet count are shown in Supplementary Table 14 
bioRxiv preprint doi: https://doi.org/10.1101/2021.04.20.440612; this version posted April 20, 2021. The copyright holder for this preprint (which

was not certified by peer review) is the author/funder, who has granted bioRxiv a license to display the preprint in perpetuity. It is made available under aCC-BY-NC-ND 4.0 International license.

\begin{tabular}{|c|c|c|c|c|c|c|}
\hline Gene & $\begin{array}{c}\text { African-American } \\
\text { (PAGE) }\end{array}$ & European & South Asian & East Asian & $\begin{array}{c}\text { Native } \\
\text { Hawaiian }\end{array}$ & $\begin{array}{c}\text { Hispanic and } \\
\text { Latin American }\end{array}$ \\
\hline$A P O A 1$ & $4.99 \times 10^{-1}$ & $1.00 \times 10^{-20}$ & $9.91 \times 10^{-1}$ & $1.00 \times 10^{-20}$ & $7.52 \times 10^{-1}$ & $4.99 \times 10^{-1}$ \\
\hline$A P O A 4$ & $4.99 \times 10^{-1}$ & $1.00 \times 10^{-20}$ & $2.51 \times 10^{-5}$ & $1.00 \times 10^{-20}$ & $9.15 \times 10^{-1}$ & $4.99 \times 10^{-1}$ \\
\hline APOA5 & $4.99 \times 10^{-1}$ & $1.42 \times 10^{-11}$ & $1.60 \times 10^{-6}$ & $9.95 \times 10^{-1}$ & $3.67 \times 10^{-12}$ & $4.99 \times 10^{-1}$ \\
\hline APOC3 & $4.99 \times 10^{-1}$ & $1.00 \times 10^{-20}$ & $9.82 \times 10^{-1}$ & $9.83 \times 10^{-1}$ & $3.05 \times 10^{-15}$ & $4.99 \times 10^{-1}$ \\
\hline$A P O E$ & $4.99 \times 10^{-1}$ & $1.00 \times 10^{-20}$ & $8.65 \times 10^{-1}$ & $1.00 \times 10^{-20}$ & 1 & 1 \\
\hline PLTP & $4.99 \times 10^{-1}$ & $4.29 \times 10^{-9}$ & $9.66 \times 10^{-1}$ & $6.66 \times 10^{-15}$ & $1.00 \times 10^{-2}$ & $4.99 \times 10^{-1}$ \\
\hline$L P L$ & $4.99 \times 10^{-1}$ & $4.08 \times 10^{-13}$ & $3.00 \times 10^{-3}$ & $1.00 \times 10^{-20}$ & $6.59 \times 10^{-1}$ & $4.99 \times 10^{-1}$ \\
\hline ANGPTL3 & $4.99 \times 10^{-1}$ & $8.86 \times 10^{-8}$ & $2.00 \times 10^{-3}$ & $1.00 \times 10^{-20}$ & $4.00 \times 10^{-3}$ & 1 \\
\hline ANGPTL4 & $4.99 \times 10^{-1}$ & $1.00 \times 10^{-20}$ & $9.78 \times 10^{-1}$ & $9.99 \times 10^{-1}$ & $9.89 \times 10^{-1}$ & 1 \\
\hline$A P O C 1$ & $4.99 \times 10^{-1}$ & $1.67 \times 10^{-16}$ & $4.99 \times 10^{-1}$ & $1.00 \times 10^{-20}$ & $9.81 \times 10^{-1}$ & $4.99 \times 10^{-1}$ \\
\hline APOC2 & $4.99 \times 10^{-1}$ & $3.57 \times 10^{-13}$ & $7.71 \times 10^{-1}$ & $1.11 \times 10^{-1}$ & $9.11 \times 10^{-1}$ & $4.99 \times 10^{-1}$ \\
\hline $\mathrm{APOC}_{4}$ & $4.99 \times 10^{-1}$ & $3.72 \times 10^{-13}$ & $7.36 \times 10^{-1}$ & $2.58 \times 10^{-14}$ & $9.73 \times 10^{-1}$ & $4.99 \times 10^{-1}$ \\
\hline$A P O B$ & $4.99 \times 10^{-1}$ & $1.00 \times 10^{-20}$ & $9.99 \times 10^{-1}$ & $7.32 \times 10^{-12}$ & $1.00 \times 10^{-3}$ & 1 \\
\hline$L M F 1$ & $9.98 \times 10^{-1}$ & $8.03 \times 10^{-7}$ & 1 & $3.21 \times 10^{-2}$ & $3.79 \times 10^{-5}$ & $9.98 \times 10^{-1}$ \\
\hline$A P O L 1$ & $4.99 \times 10^{-1}$ & $5.30 \times 10^{-2}$ & $6.40 \times 10^{-2}$ & 1 & $8.89 \times 10^{-11}$ & $4.99 \times 10^{-1}$ \\
\hline$H B A 1$ & $4.99 \times 10^{-1}$ & $3.75 \times 10^{-5}$ & $9.99 \times 10^{-1}$ & $4.51 \times 10^{-1}$ & $2.46 \times 10^{-10}$ & 1 \\
\hline$H B A 2$ & $4.99 \times 10^{-1}$ & $1.30 \times 10^{-5}$ & $9.99 \times 10^{-1}$ & $4.51 \times 10^{-1}$ & $3.93 \times 10^{-10}$ & $4.99 \times 10^{-1}$ \\
\hline B4GALT3 & $4.99 \times 10^{-1}$ & $6.80 \times 10^{-2}$ & $7.21 \times 10^{-1}$ & $4.99 \times 10^{-1}$ & $1.23 \times 10^{-6}$ & 1 \\
\hline$K L K 8$ & 1 & 1 & $1.62 \times 10^{-6}$ & $9.89 \times 10^{-1}$ & $1.00 \times 10^{-3}$ & 1 \\
\hline PNLIP & $4.99 \times 10^{-1}$ & $9.99 \times 10^{-1}$ & $9.26 \times 10^{-1}$ & $7.75 \times 10^{-1}$ & $1.00 \times 10^{-3}$ & $4.99 \times 10^{-1}$ \\
\hline WNT4 & $4.99 \times 10^{-1}$ & $9.61 \times 10^{-1}$ & $9.99 \times 10^{-1}$ & $4.99 \times 10^{-1}$ & $3.29 \times 10^{-5}$ & $4.99 \times 10^{-1}$ \\
\hline$B A C E 1$ & $4.99 \times 10^{-1}$ & $5.55 \times 10^{-17}$ & $2.20 \times 10^{-2}$ & $9.99 \times 10^{-16}$ & $6.69 \times 10^{-1}$ & $4.99 \times 10^{-1}$ \\
\hline CETP & $4.99 \times 10^{-1}$ & $1.00 \times 10^{-3}$ & $9.99 \times 10^{-1}$ & $1.41 \times 10^{-6}$ & $9.99 \times 10^{-1}$ & $4.99 \times 10^{-1}$ \\
\hline PCSK6 & $4.99 \times 10^{-1}$ & 1 & $9.99 \times 10^{-1}$ & $1.83 \times 10^{-5}$ & $1.00 \times 10^{-3}$ & $4.99 \times 10^{-1}$ \\
\hline PCSK 7 & $4.99 \times 10^{-1}$ & $1.66 \times 10^{-8}$ & $9.97 \times 10^{-1}$ & $1.00 \times 10^{-20}$ & $9.99 \times 10^{-1}$ & $4.99 \times 10^{-1}$ \\
\hline$L C A T$ & $4.99 \times 10^{-1}$ & $5.00 \times 10^{-1}$ & 1 & $6.24 \times 10^{-3}$ & $4.38 \times 10^{-1}$ & $4.99 \times 10^{-1}$ \\
\hline$A P O F$ & $4.99 \times 10^{-1}$ & $5.78 \times 10^{-1}$ & $7.79 \times 10^{-1}$ & $4.10 \times 10^{-3}$ & $9.64 \times 10^{-1}$ & $4.99 \times 10^{-1}$ \\
\hline TYRO3 & $4.99 \times 10^{-1}$ & $9.28 \times 10^{-1}$ & $9.99 \times 10^{-1}$ & $1.20 \times 10^{-2}$ & $8.57 \times 10^{-1}$ & $4.99 \times 10^{-1}$ \\
\hline
\end{tabular}

Supplementary Table 18: Gene- $\varepsilon p$-values for the 28 genes present in the significantly mutated sunnetworks associated with triglyceride level in the European, East Asian, and Native Hawaiian cohorts. Each of these genes is present in Figure 3 which depicts the overlapping significantly mutated subnetworks identified using Hierarchical HoNet ${ }^{61}$ identified in an anlaysis of triglyceride levels in the European, East Asian, and Native Hawaiian cohorts. Known SNP-level associations identified within the bounds of these genes in previous studies submitted to the GWAS Catalog are discussed in the Supplementary Note. Ancestry-specific Bonferroni corrected significance thresholds for gene-level association analysis of triglyceride levels are shown in Supplementary Table 14. 


\section{Supplementary Figures}

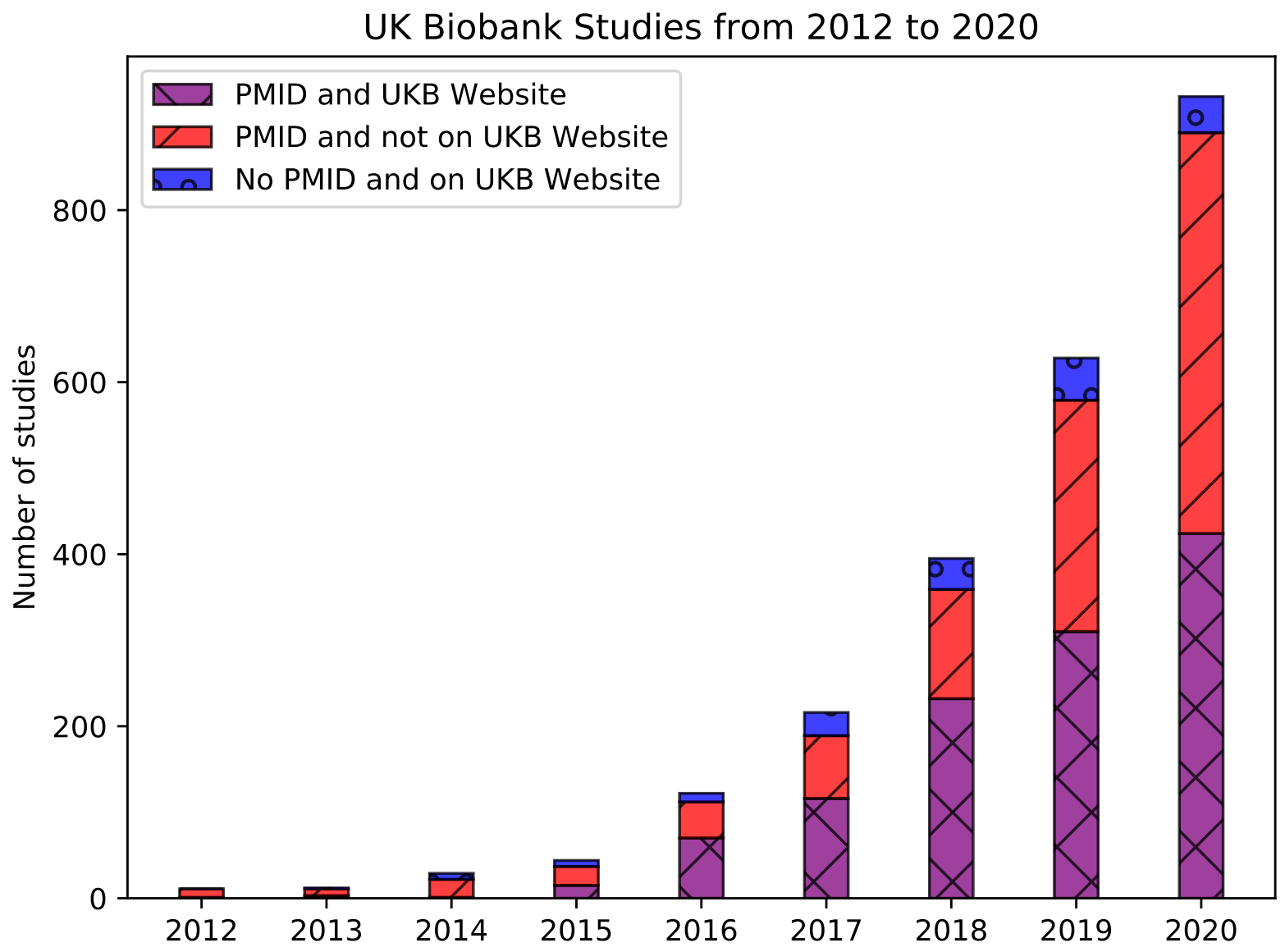

Supplementary Figure 1: Number of publications we identified using UK Biobank data from 2012 to 2020. Studies identified using PMIDs as described in the Online Methods section. Studies that are displayed on the UK Biobank website (https://www.ukbiobank.ac.uk/) and identified on PubMed are shown in purple. Studies listed on the UK Biobank website but do not have a PMID are shown in blue, and studies only identified using PubMed but not listed on the UK Biobank website are shown in red. The protocols for identifying studies both on PubMed and the UK Biobank website are detailed in the Online Methods section. Data from both the UK Biobank website and PubMed were accessed on January 12, 2021. 


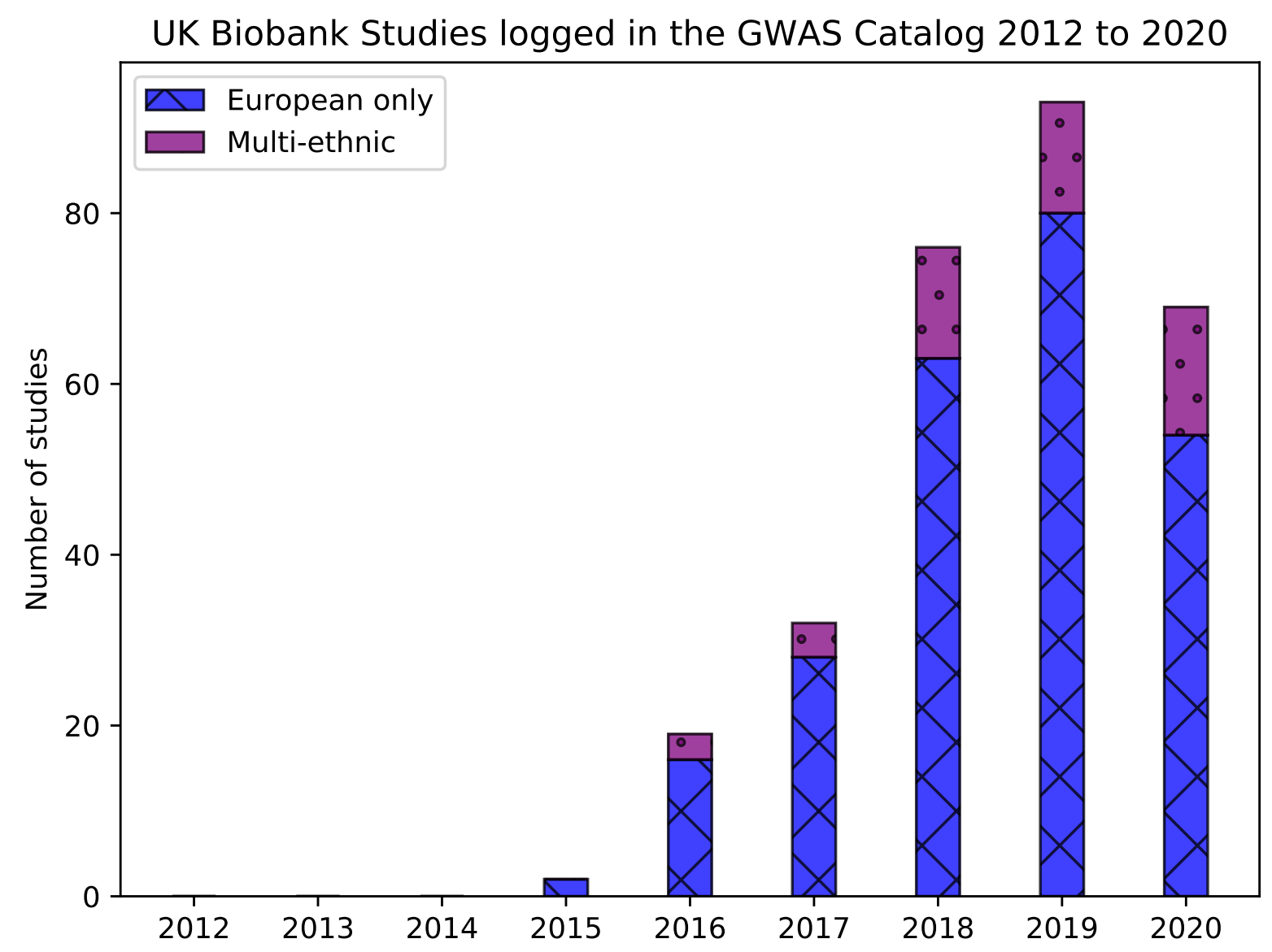

Supplementary Figure 2: Number of studies published using UK Biobank data from 2012 to 2020 that have available metadata in the GWAS Catalog. Our protocols for identifying studies from the GWAS Catalog are detailed in the Online Methods section. Multi-ancestry studies are shown in purple and include those that list samples of more than one ancestral group in the GWAS catalog (as defined according to the protocol using Popejoy and Fullerton ${ }^{25}$, available on the GitHub page https: //github.com/ramachandran-lab/redefining_replication). Studies that only list samples of European ancestry in the GWAS catalog are shown in blue. Every multi-ancestry analysis includes samples of European ancestry and of at least one other ancestry. GWAS Catalog data was accessed on January 10, 2021 from the website https://www.ebi.ac.uk/gwas/docs/file-downloads using the final release file of 2020 (see file named gwas_catalog_v1.0.2-associations_e100_r2020-12-15.tsv). 
bioRxiv preprint doi: https://doi.org/10.1101/2021.04.20.440612; this version posted April 20, 2021. The copyright holder for this preprint (which was not certified by peer review) is the author/funder, who has granted bioRxiv a license to display the preprint in perpetuity. It is made available under aCC-BY-NC-ND 4.0 International license.

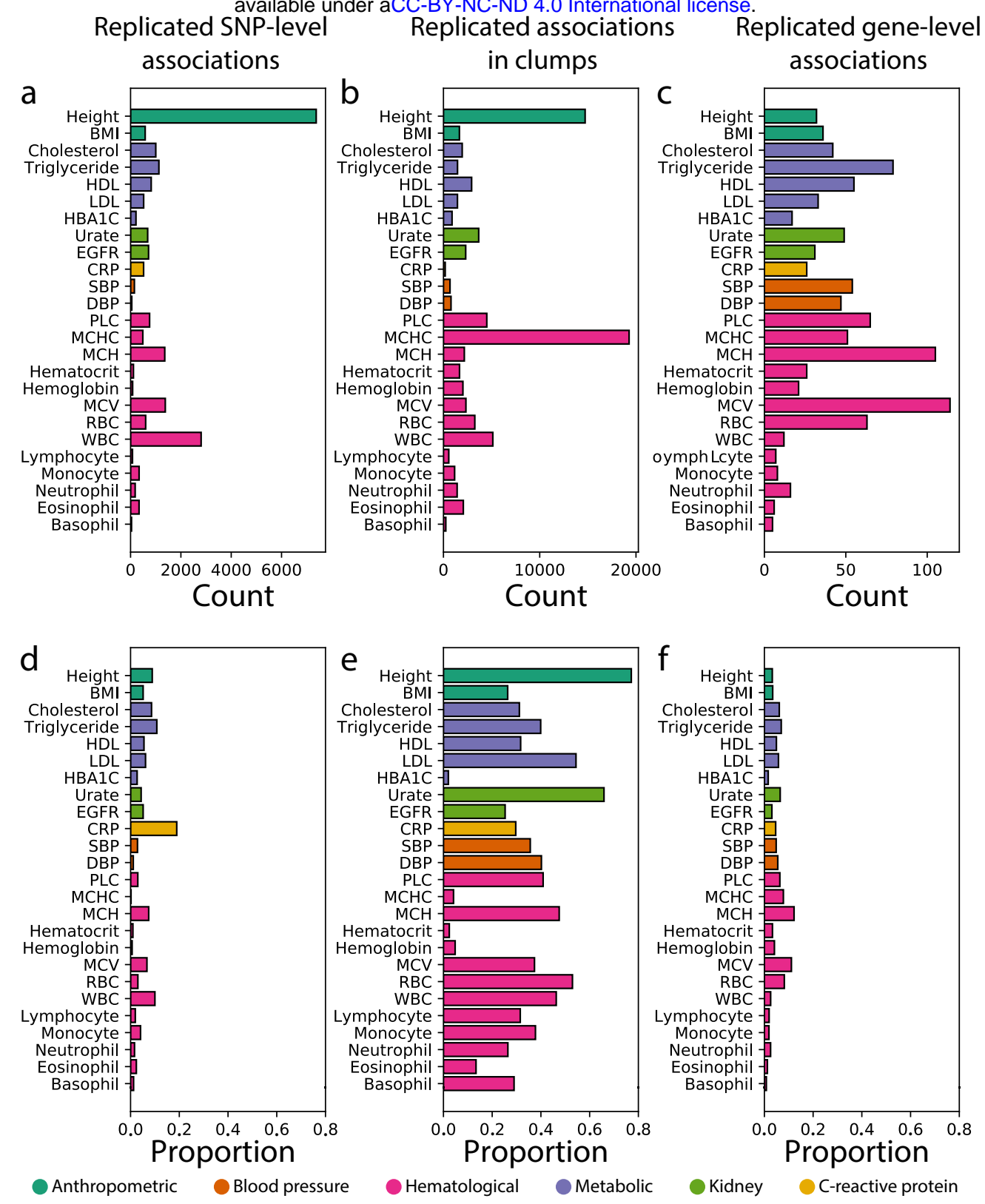

Supplementary Figure 3: Summaries of replicated associations at multiple genomic scales among ancestry cohorts for all 25 traits analyzed using Bonferroni-corrected thresholds. Expansion of three letter trait codes are given in Supplementary Table 2. (a) Number and (d) proportion of genome-wide significant SNPs associated with a phenotype in at least one ancestry cohort that were replicated in at least two ancestry cohorts. In all 25 traits, genome-wide significant SNPs replicate in at least two ancestry cohorts. Height contains over 7,000 replicated SNPs among the seven ancestry cohorts analyzed, illustrating its highly polygenic architecture. For many traits across all categories, with the exception of other biochemical (i.e., CRP), the replication rate of genes is higher in gene-level associations than at the SNP-level. (b) Number and (e) proportion of $1 \mathrm{Mb}$ windows, or "clumps", that contain at least one genomewide significant SNP-level associations for a given phenotype in at least two ancestry cohorts. (c) Number and (f) proportion of genome-wide significant gene-level associations that replicate among ancestry cohorts. Replicated associations in hematological are common at the gene-level in hematological and metabolic traits. For instance, in three of the four cohorts with mean corpuscular hemoglobin (MCH) measurements HBA1 and HBA2 were identified as significant associated with $\mathrm{MCH}$ in the African, European, and East Asian ancestry cohorts Supplementary Table 3. The denominator of the proportion is calculated as the total number of unique SNPs, clumps, or genes that are significantly associated with a trait in at least on ancestral cohort. Note that $\mathbf{d}$ and $\mathbf{f}$ correspond to Figure $1 \mathbf{a}$ and $\mathbf{b}$, with an altered $\mathrm{x}$-axis upper limit of 0.8 in this figure. 
a

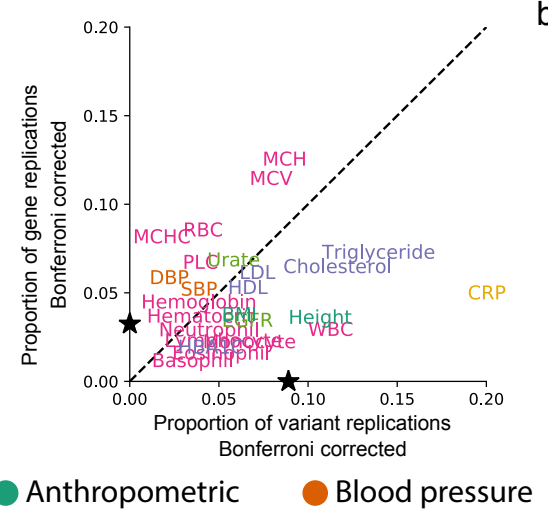

b

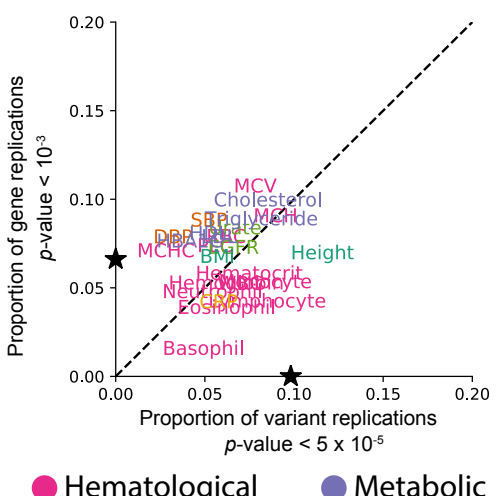

C

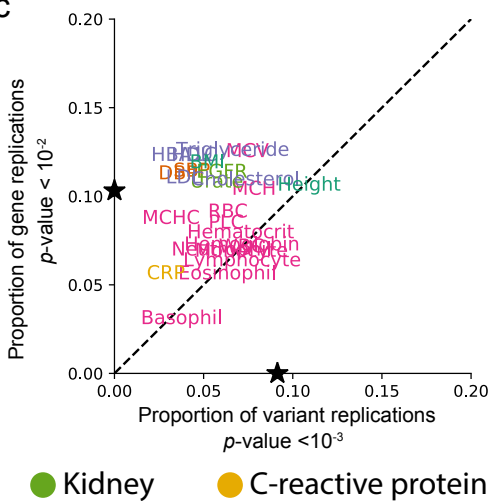

Supplementary Figure 4: Less stringent significance thresholds lead to a decrease in the proportion of replicated SNP-level associations and an increase in the proportion of gene-level associations among ancestries for each of the 25 traits analyzed. a. Proportion of all SNP-level Bonferroni-corrected genome-wide significant associations in any ancestry that replicate in at least one other ancestry is shown on the $\mathrm{x}$-axis (see Supplementary Table 10 for ancestry-trait specific Bonferroni corrected $p$-value thresholds). On the $\mathrm{y}$-axis we show the proportion of significant gene-level associations that were replicated for a given phenotype in at least two ancestries (see Supplementary Table 14 for Bonferroni corrected significance thresholds for each ancestry-trait pair). The black stars on the $\mathrm{x}$ - and $\mathrm{y}$-axes represent the mean proportion of replicates in SNP and gene analyses, respectively. C-reactive protein (CRP) contains the greatest proportion of replicated SNP-level associations of any of the phenotypes. $\mathbf{b}$. The $\mathbf{x}$-axis indicates the proportion of SNP-level associations that surpass a nominal threshold of $p$-value $<10^{-5}$ in at least one ancestry cohort that replicate in at least one other ancestry cohort. The y-axis indicates the proportion of gene-level associations that surpass a nominal threshold of $p$-value $<10^{-3}$ in at least one ancestry cohort and replicate in at least one other ancestry cohort. Nominal $p$-value thresholds tend to decrease the proportion of replicated SNP-level associations and tend to increase the proportion of replicated gene-level associations. The number of unique SNPs and genes that replicated in each cohort is given in Supplementary Figure 5. c. The $\mathrm{x}$-axis indicates the proportion of SNP-level associations that surpass a nominal threshold of $p$-value $<10^{-3}$ in at least one ancestry cohort that replicate in at least one other ancestry cohort. The $\mathrm{y}$-axis indicates the proportion of gene-level associations that surpass a nominal threshold of $p$-value $<10^{-2}$ in at least one ancestry cohort and replicate in at least one other ancestry cohort. The number of unique SNPs and genes that replicated in each cohort is given in Supplementary Figure 6 As shown in panel $\mathbf{b}$, nominal $p$-value thresholds tend to decrease the proportion of replicated SNP-level associations and tend to increase the proportion of replicated gene-level associations. Expansion of three letter trait codes are given in Supplementary Table 2. 
bioRxiv preprint doi: https://doi.org/10.1101/2021.04.20.440612; this version posted April 20, 2021. The copyright holder for this preprint (which was not certified by peer review) is the author/funder, who has granted bioRxiv a license to display the preprint in perpetuity. It is made available under aCC-BY-NC-ND 4.0 International license.

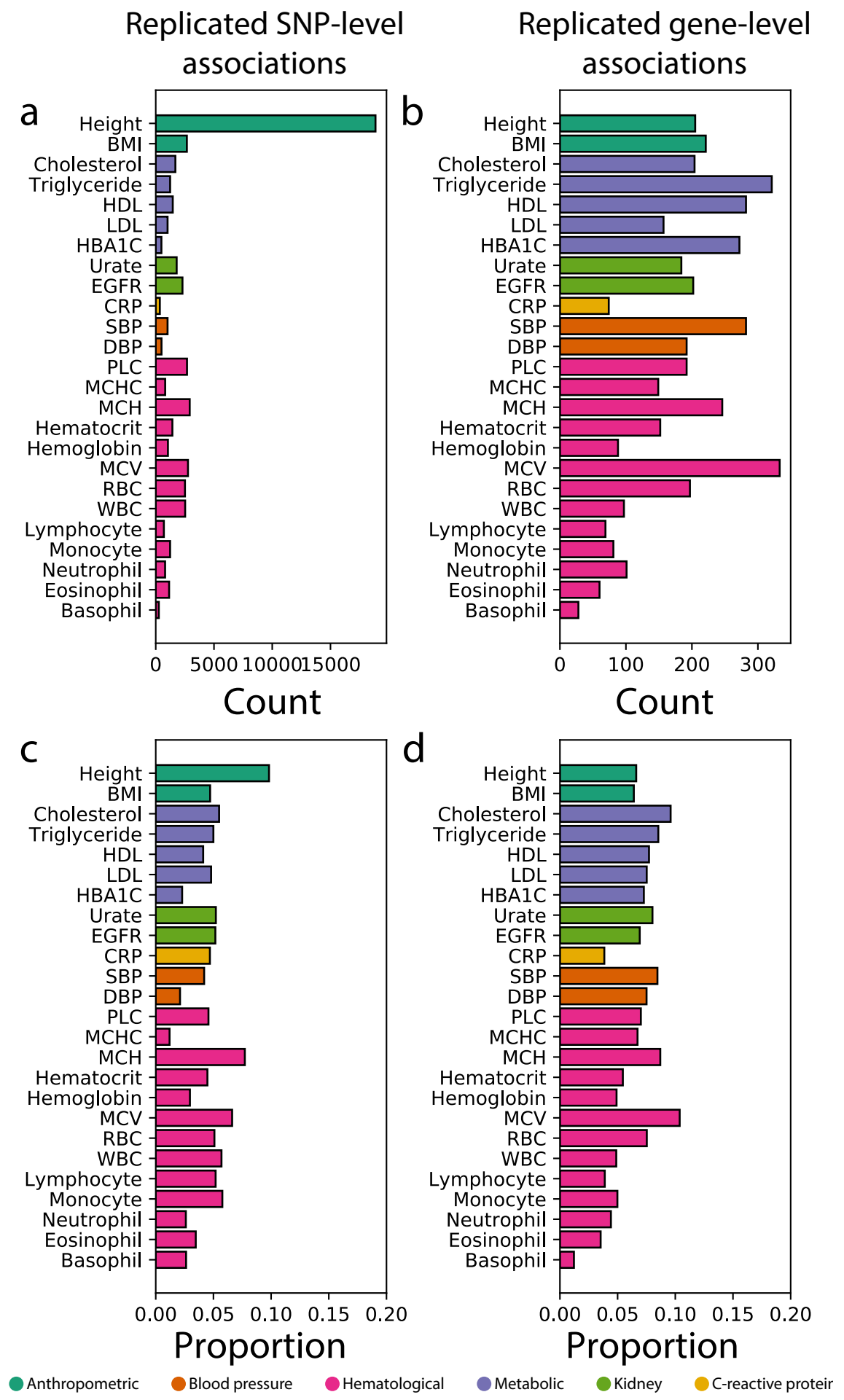

Supplementary Figure 5: Summaries of replicated associations at multiple genomic scales among ancestry cohorts for all 25 traits analyzed using nominal $p$-value thresholds. (a) Number and (c) proportion of genome-wide significant SNPs associated with a phenotype in at least one ancestry cohort that were replicated in at least two ancestry cohorts using a nominal $p$-value threshold of $5 \times 10^{-5}$. (b) Number and (d) proportion of genome-wide significant gene-level associations that replicate among ancestry cohorts using a nominal $p$-value threshold of $10^{-3}$. Expansion of three letter trait codes are given in Supplementary Table 2. 
bioRxiv preprint doi: https://doi.org/10.1101/2021.04.20.440612; this version posted April 20, 2021. The copyright holder for this preprint (which was not certified by peer review) is the author/funder, who has granted bioRxiv a license to display the preprint in perpetuity. It is made available under aCC-BY-NC-ND 4.0 International license.
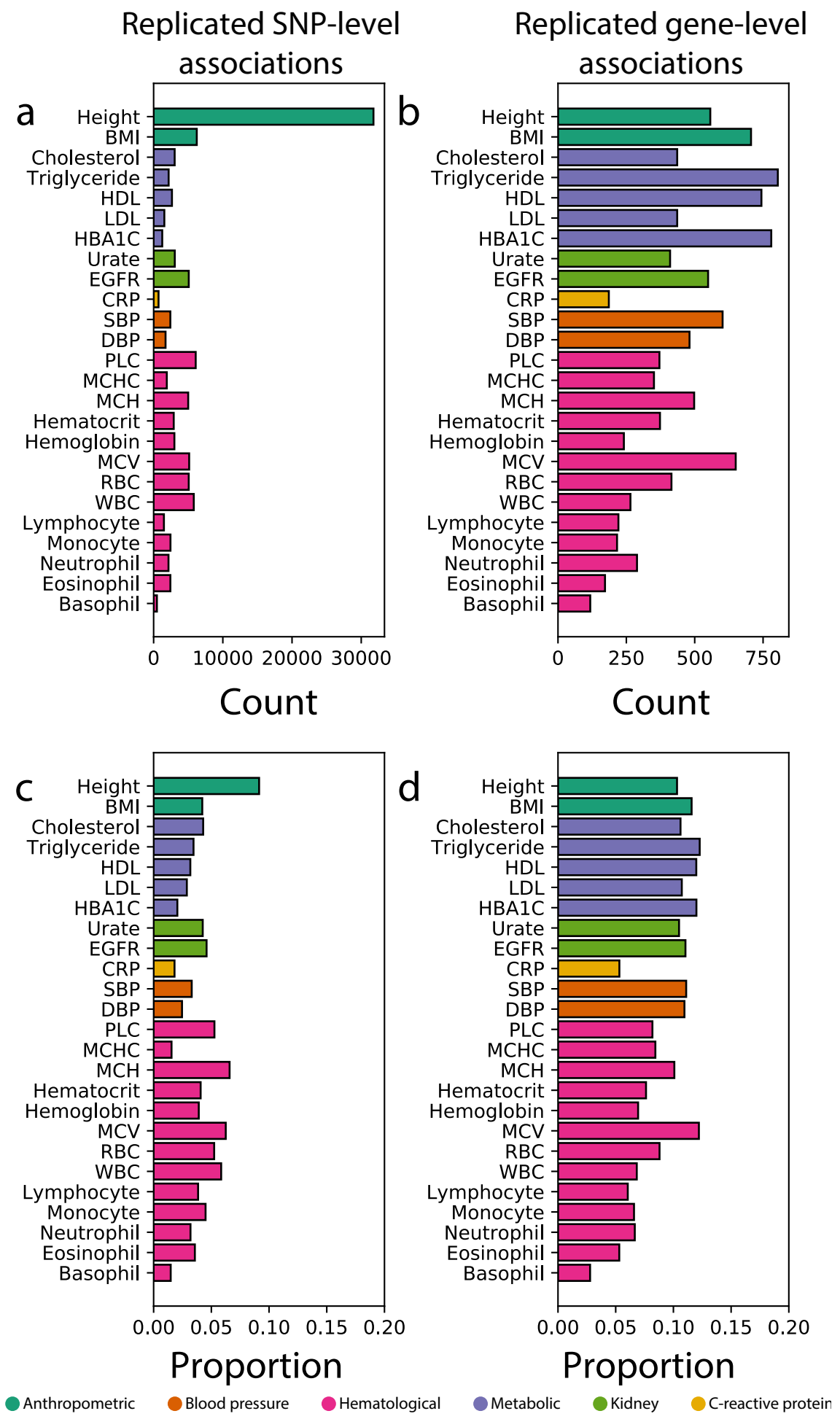

Supplementary Figure 6: Summaries of replicated associations at multiple genomic scales among ancestry cohorts for all 25 traits analyzed using nominal $p$-value thresholds. (a) Number and (c) proportion of genome-wide significant SNPs associated with a phenotype in at least one ancestry cohort that were replicated in at least two ancestry cohorts using a nominal $p$-value threshold of $10^{-3}$. (b) Number and (d) proportion of genome-wide significant gene-level associations that replicate among ancestry cohorts using a nominal $p$-value threshold of $10^{-2}$. Expansion of three letter trait codes are given in Supplementary Table 2. Expansion of three letter trait codes are given in Supplementary Table 2. 


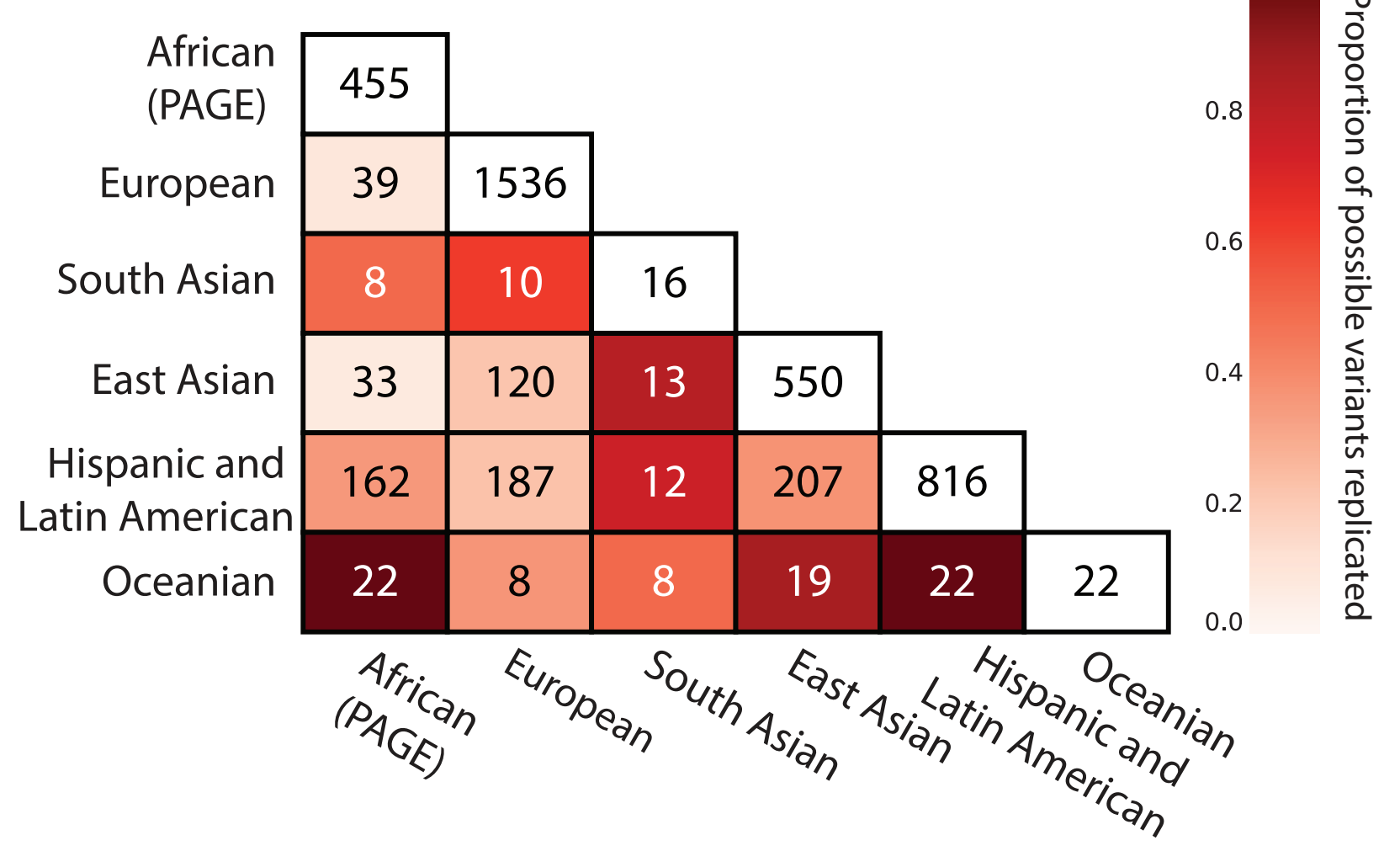

Supplementary Figure 7: Pairwise replication of SNP-level association signals for C-reactive protein in six ancestral cohorts using genotype and imputed data. Imputed data was available and included in this analysis for each of the six cohorts. The inclusion of imputed SNPs in GWA analysis of C-reactive protein increases both the number of significant SNPs in each ancestry (along the diagonal) as well as the number of replicating significant SNP-level associations among pairs of ancestry cohorts (lower triangular). The corresponding analysis of pairwise SNP-level replication using only genotype data from each cohort is shown in Figure $2 \mathrm{c}$. 


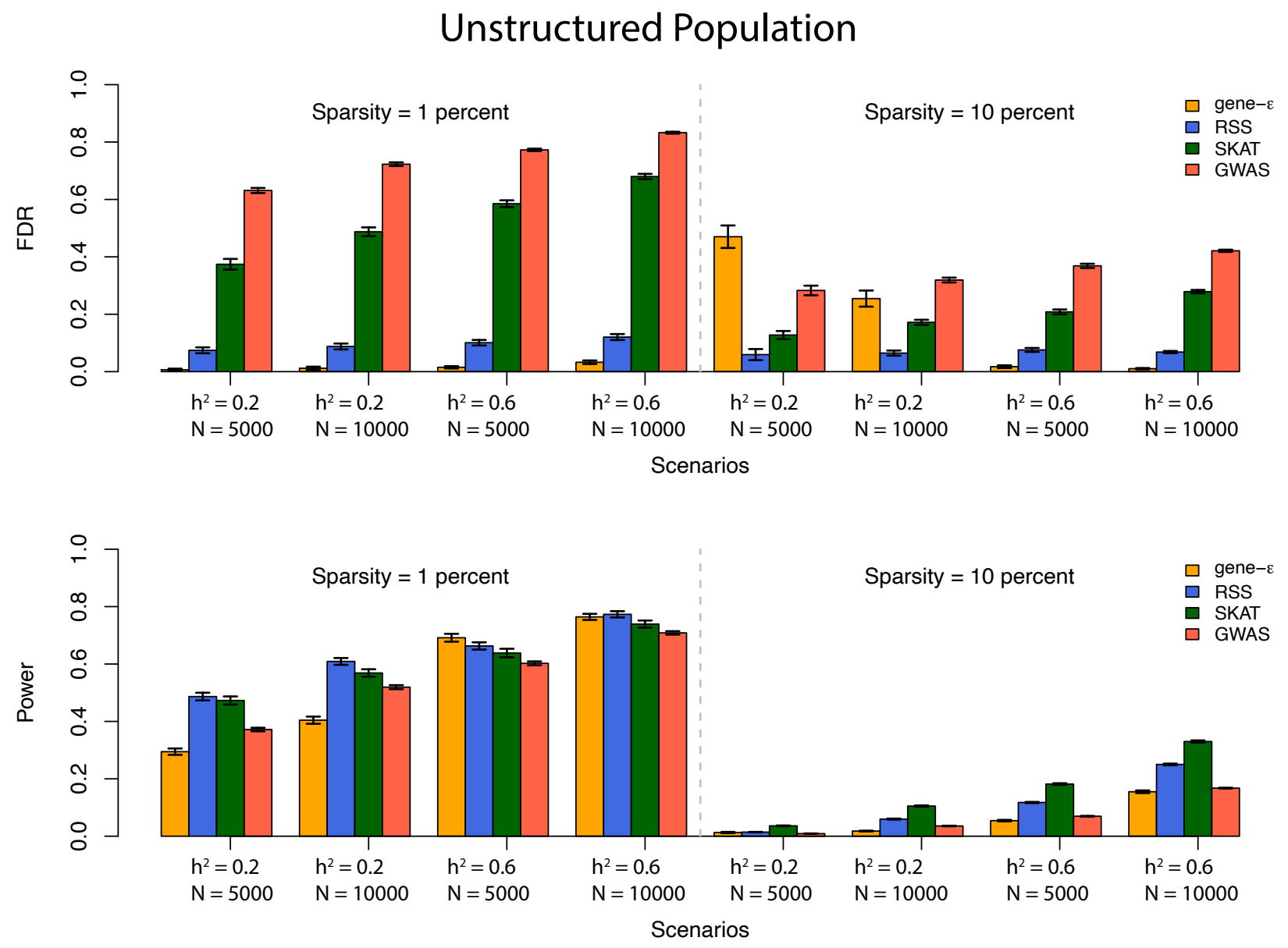

Supplementary Figure 8: gene- $\varepsilon$ outperforms and controls false discovery rate (FDR) better than other association methods in simulations with varying heritability and sample size. Simulations were designed to assess gene versus SNP-level association false discovery rate (FDR) and power in an unstructured population as described by the protocols in the Online Methods section. The top and bottom panels show the FDR and power of four different association methods on 100 simulated datasets, respectively. We compared performance of three gene-level association test methods (gene- $\varepsilon^{53}$, RSS $\frac{52}{52}$, SKAT $\frac{48}{4}$ ) with outputs from the standard GWA association test under different simulation parameters (sample size $N$, narrow-sense heritability $h^{2}$, and sparsity). We define sparsity as the proportion of SNPs that are groundtruth causal. Standard errors across the simulated replicates are shown using black whisker plots. Simulation protocol is described in the Online Methods section. 


\section{Structured Population}
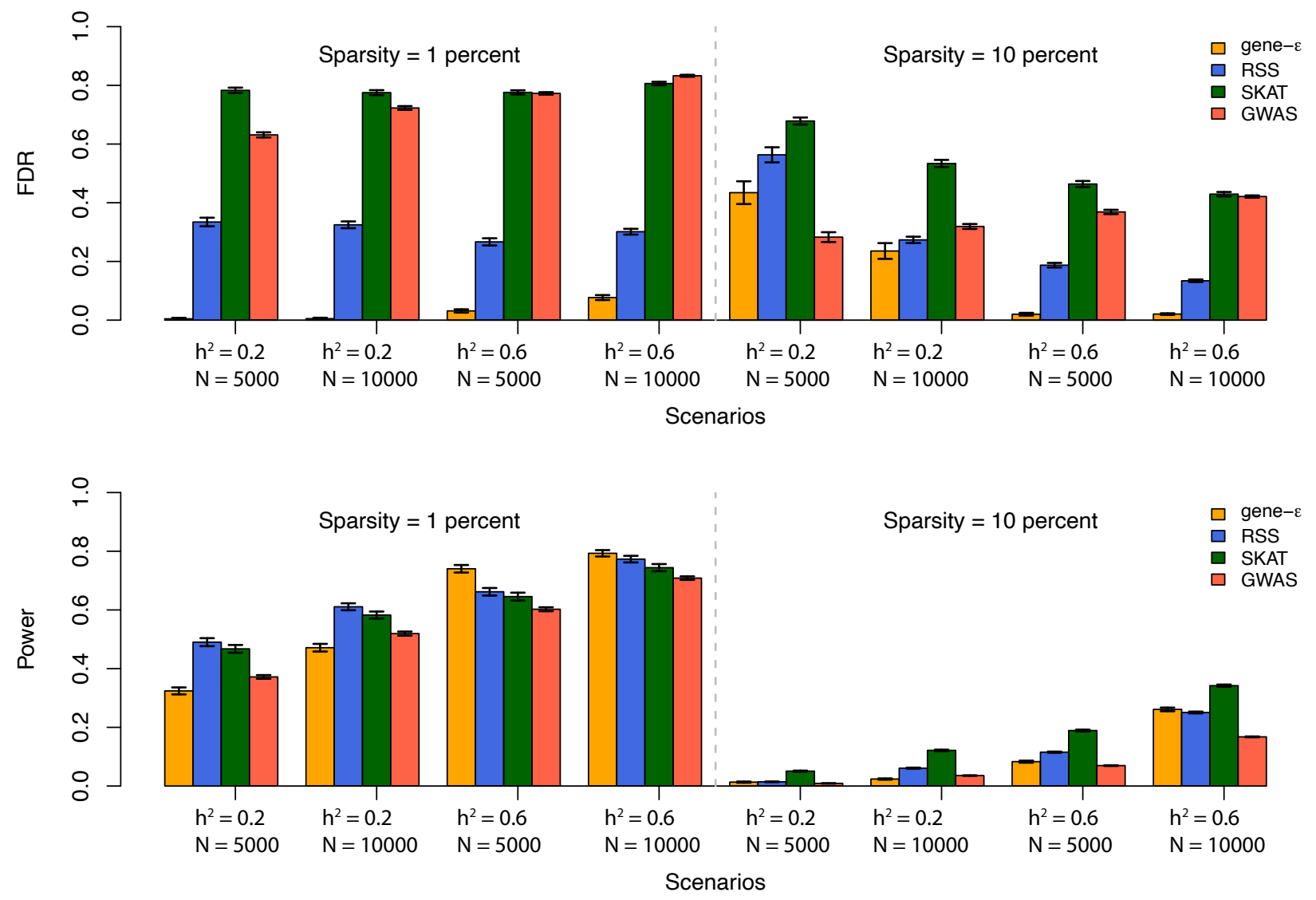

Supplementary Figure 9: gene- $\varepsilon$ outperforms and controls false discovery rate (FDR) better than other association methods in simulations with varying heritability and sample size. Simulations are designed to assess gene versus SNP-level association false discovery rate (FDR) and power in an structured population as described by the protocols in the Online Methods section. The top and bottom panels show the FDR and power of four different association methods on 100 simulated datasets, respectively. We compared performance of three gene-level association test methods (gene- $\varepsilon^{53}$, RSS $\frac{52}{2}$, SKAT ${ }^{[48}$ ) with outputs from the standard GWA association test under different simulation parameters (sample size $N$, narrow-sense heritability $h^{2}$, and sparsity). We define sparsity as the proportion of SNPs that are designated to be causal. Standard errors across the simulated replicates are shown using black whisker plots. 


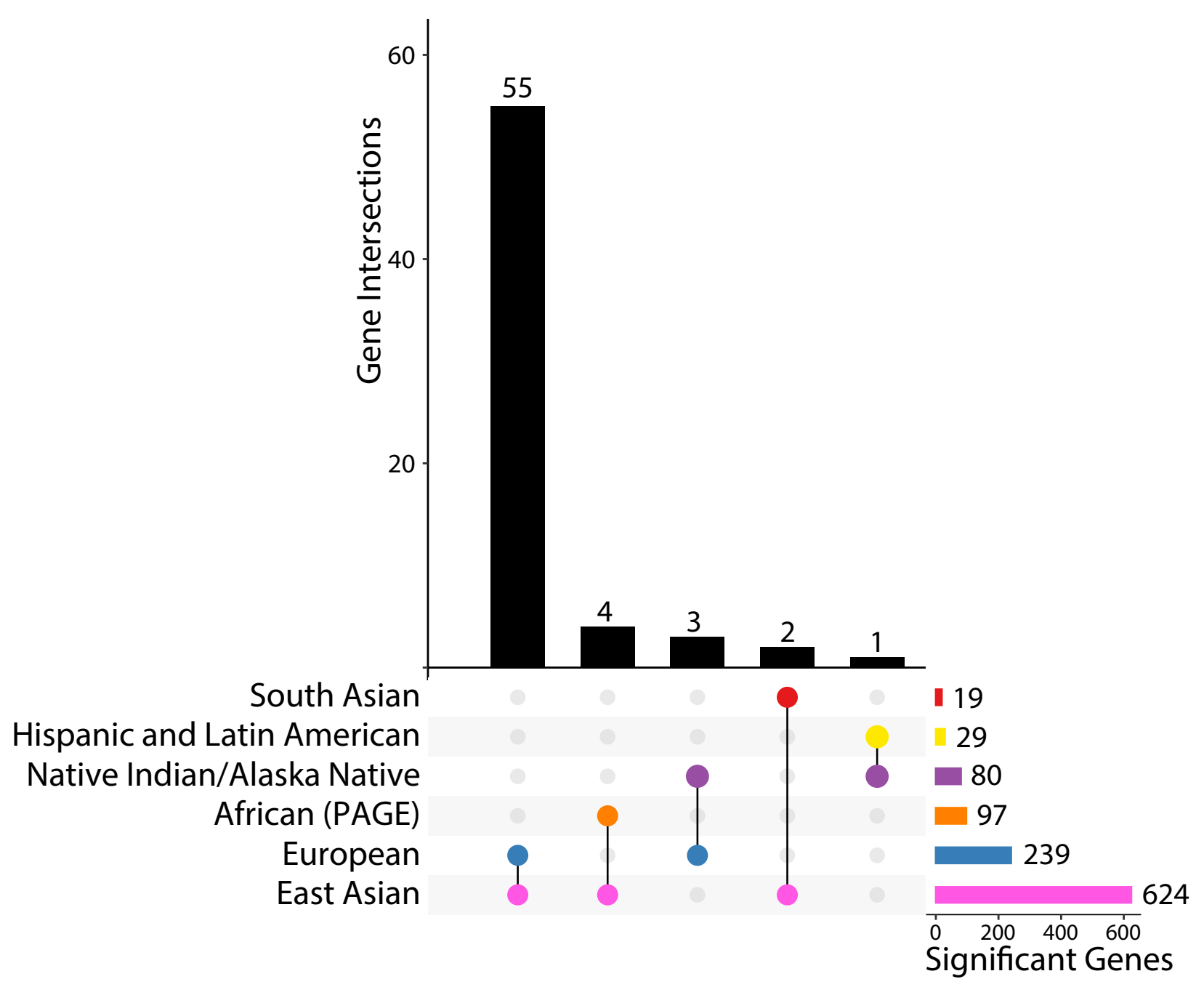

Supplementary Figure 10: All six ancestries have gene-level associations with platelet count that replicate in at least one other ancestry. Total number of genome-wide significant genes in each ancestry, after correcting for total number of regions tested, are given in the bar plot located in the bottom right (significance thresholds are given in Supplementary Table 14, sample sizes are given in Supplementary Table 5 - Supplementary Table 8). Shared gene-level association statistics between pairs of ancestries are shown in the vertical bar plot; the pair of ancestries represented by each bar can be identified using the dots and links below the barplot. Of the 65 genome-wide significant gene-level association statistics that replicate in at least two ancestry cohorts, only 25 contain SNPs that have been previously associated with platelet count in at least one ancestry in at least one study in the GWAS catalog (https://www.ebi.ac.uk/gwas/home) This plot was generated using the UpSetR package ${ }^{122}$. 

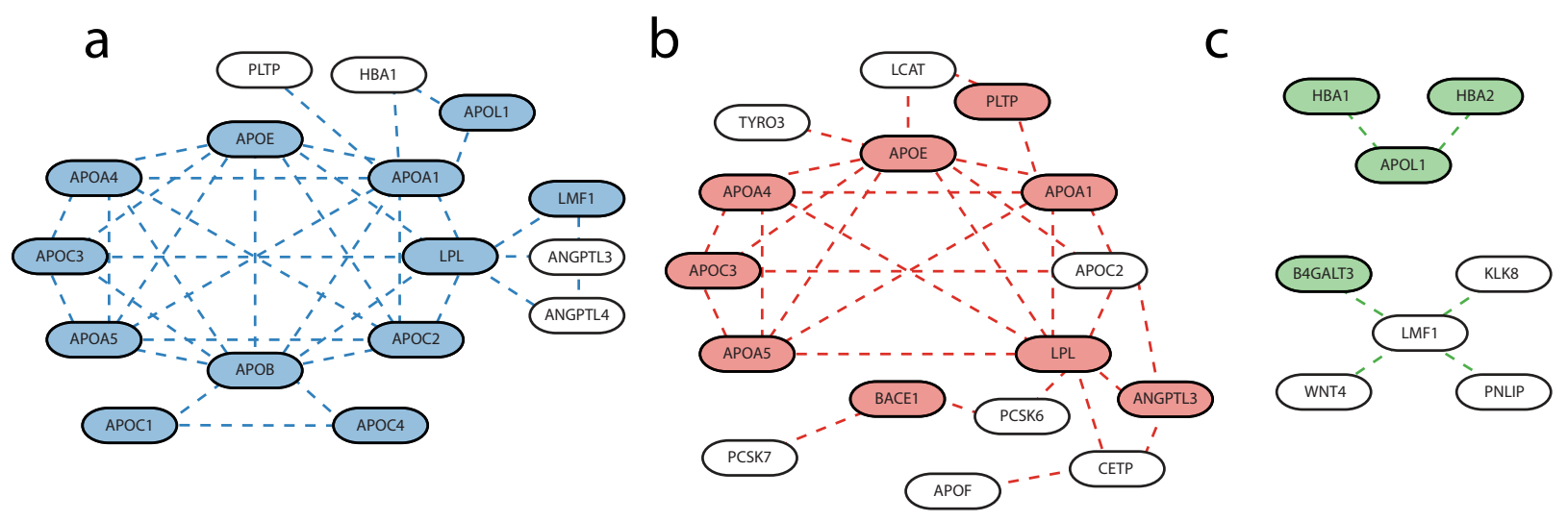

Supplementary Figure 11: Significantly mutated subnetworks associated with triglyceride levels identified in the (a) European, (b) East Asian, and (c) Native Hawaiian ancestry cohorts. Significantly mutated subnetworks were identified using the Hierarchical HotNet method ${ }^{61}$. Genes that were identified in each ancestry as significantly associated with triglyceride levels using the gene- $\varepsilon$ method are shaded using ancestry-specific color coding (also used in Figure 3, European — blue, East Asian — pink, Native Hawaiian - green). Significantly mutated subnetworks in the (a) European and (b) East Asian cohorts were identified using the ReactomeFI ${ }^{94}$ protein-protein interaction network, and the significantly mutated subnetwork in the (c) the Native Hawaiian cohort was identified using the iRefIndex $15.0^{95}$ protein-protein interaction networks. Genes that are present in any of the significantly mutated subnetworks that contain SNPs previously associated with triglyceride levels in the GWAS Catalog are listed with corresponding citations in Supplementary Table 15. 


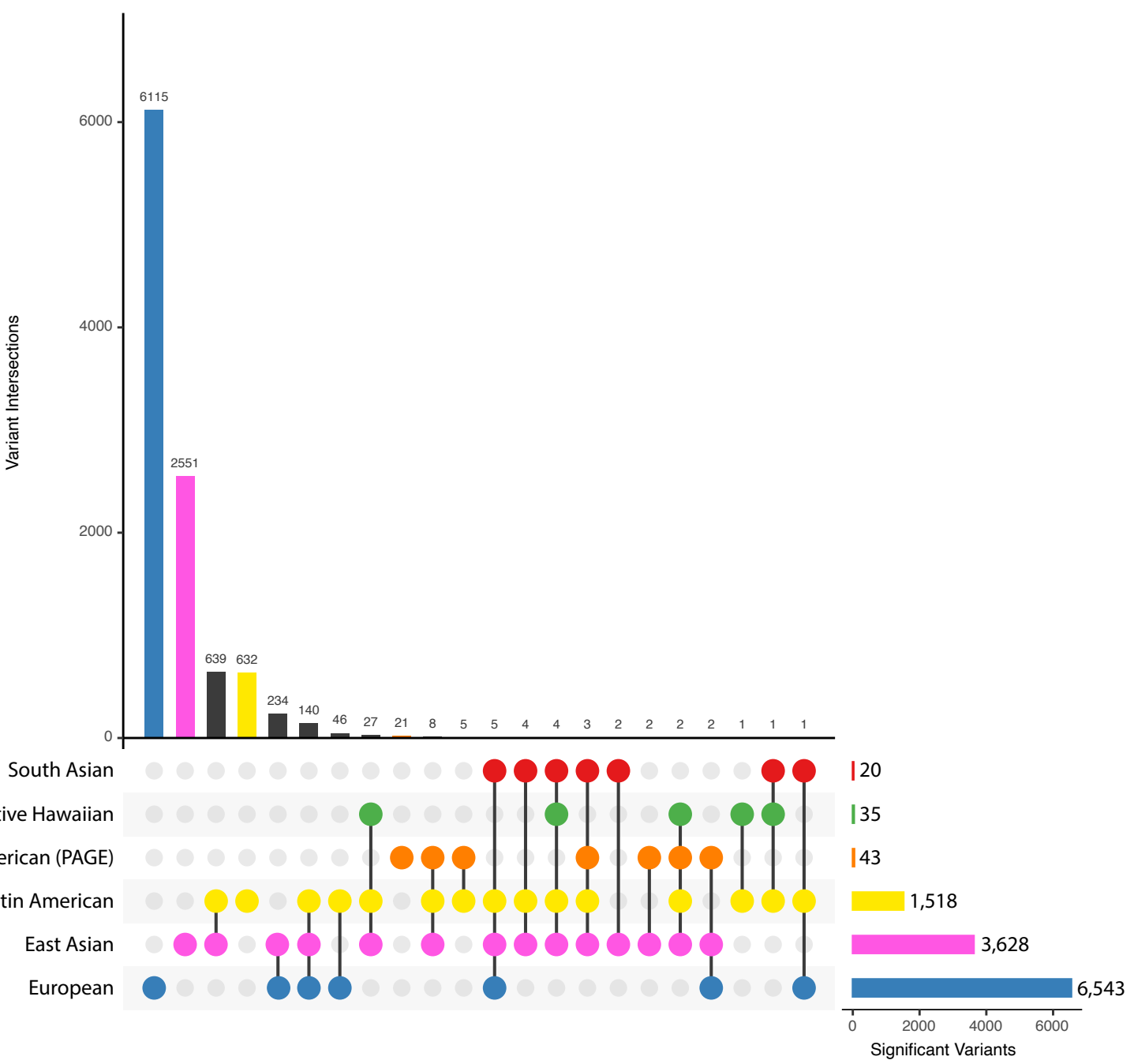

Supplementary Figure 12: Shared SNP-level associations with triglyceride levels in six ancestral cohorts. Total number of genome-wide significant genes in each ancestry, after correcting for total number of regions tested, are given in the bar plot located in the bottom right (significance thresholds are given in Supplementary Table 10 and sample sizes are given in Supplementary Table 5 - Supplementary Table 8). Shared SNP-level association statistics between pairs of ancestries are shown in the vertical bar plot. The pair of ancestries represented by each bar can be identified using the dots and links below the vertical barplot. This plot was generated using the UpSetR package ${ }^{122}$. 


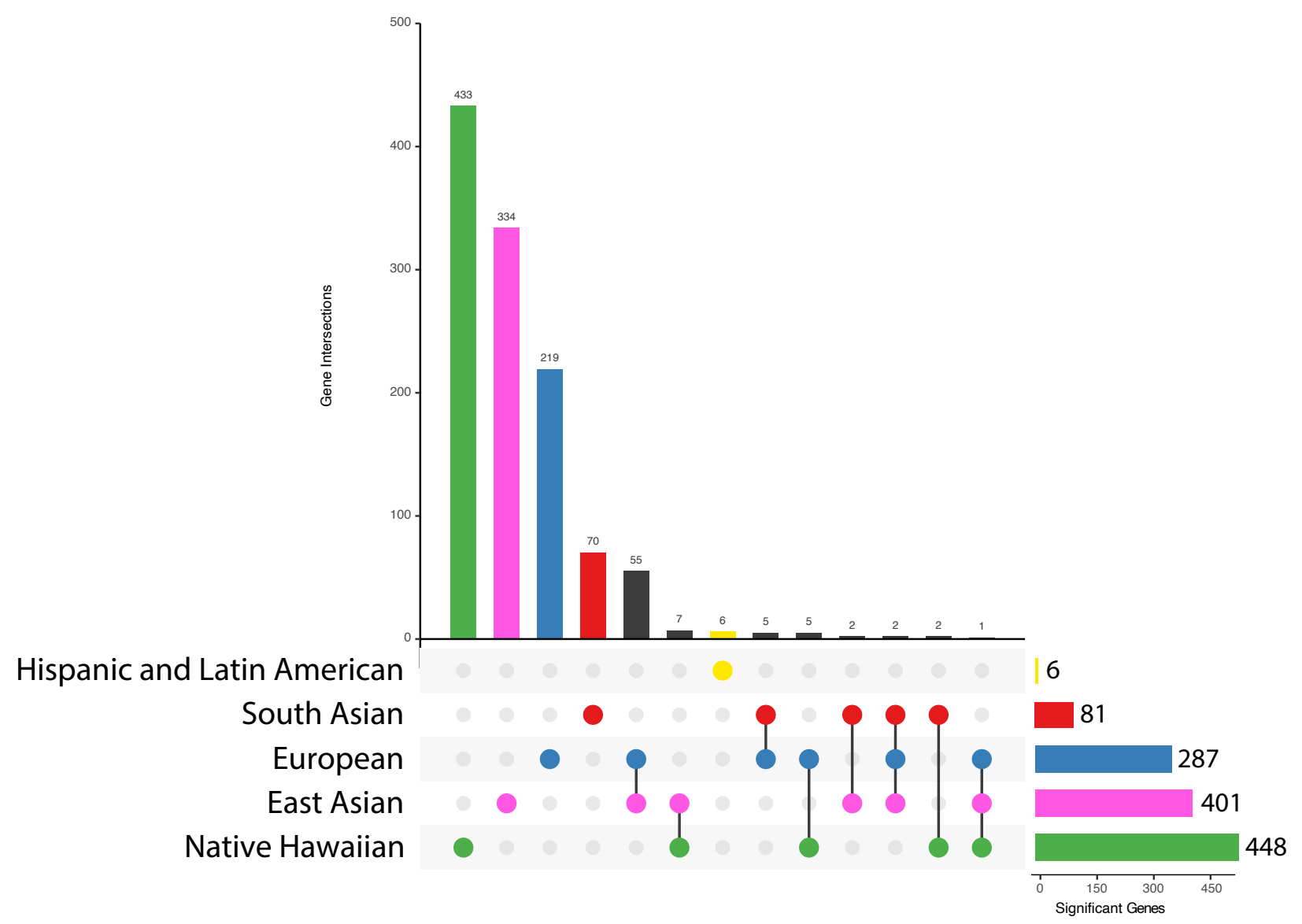

Supplementary Figure 13: Shared gene-level associations with triglyceride levels in five ancestral cohorts. Total number of genome-wide significant genes in each ancestry, after correcting for total number of regions tested, are given in the bar plot located in the bottom right (significance thresholds are given in Supplementary Table 14 and sample sizes are given in Supplementary Table 5 - Supplementary Table 8). Shared gene-level association statistics between pairs of ancestries are shown in the vertical bar plot. The pair of ancestries represented by each bar can be identified using the dots and links below the vertical barplot. This plot was generated using the UpSetR package ${ }^{122}$. 

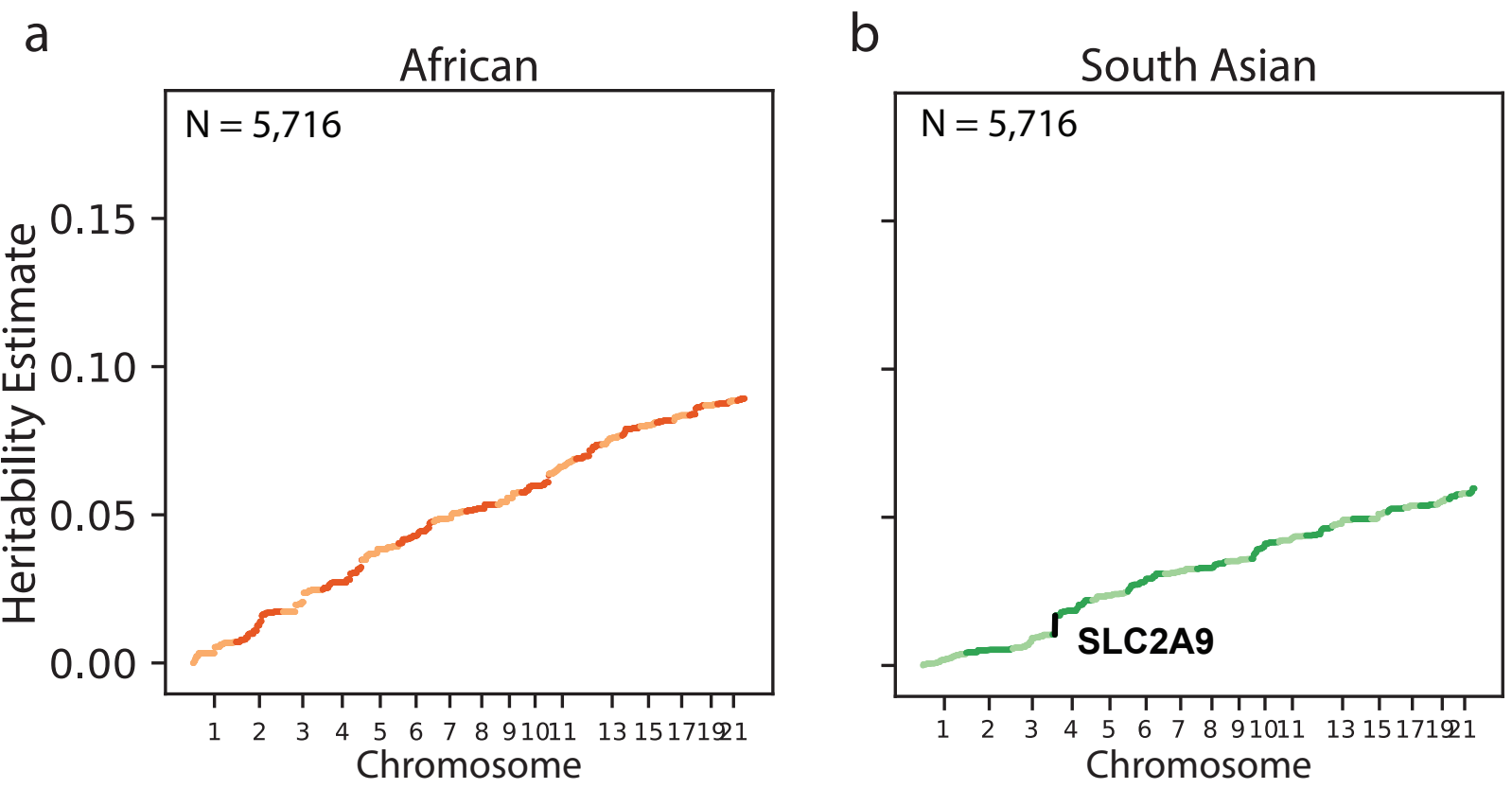

Supplementary Figure 14: A single region is identified as explaining a significant amount of narrow-sense heritability for urate levels in the South Asian ancestry cohort. Narrow-sense heritability estimates for each ancestry were obtained using HESS ${ }^{66}$. The region containing known urate transporter SLC2A9, is also identified in our analysis of the South Asian ancestry cohort. 


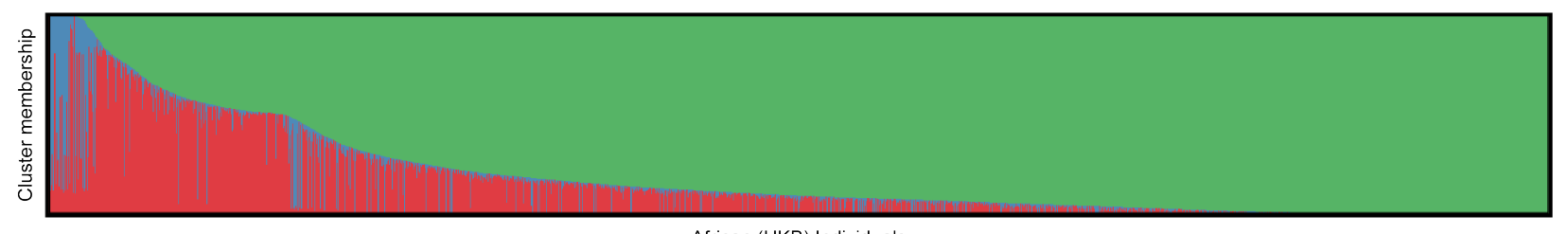

African (UKB) Individuals

Supplementary Figure 15: ADMIXTURE results from self-identified African individuals in the UK Biobank. We performed 10 runs of unsupervised ADMIXTURE ${ }^{96}$ setting $K=3$ on 4,967 selfidentified African individuals in the UK Biobank. We included YRI and CEU individuals from the 1000 Genomes Project $\frac{101}{10}$ to identify European and African ancestry components in the ADMIXTURE results. We then filtered out all individuals with less than $5 \%$ membership in the African component (identified as the component shown in green). The AIAN and European ancestry components are shown in blue and red, respectively. Our ADMIXTURE pipeline used the same protocol described in Bitarello and Mathieson 82 . All scripts for ADMIXTURE runs as well as filtration steps are available on the GitHub page given in the Data Availability section. 
a

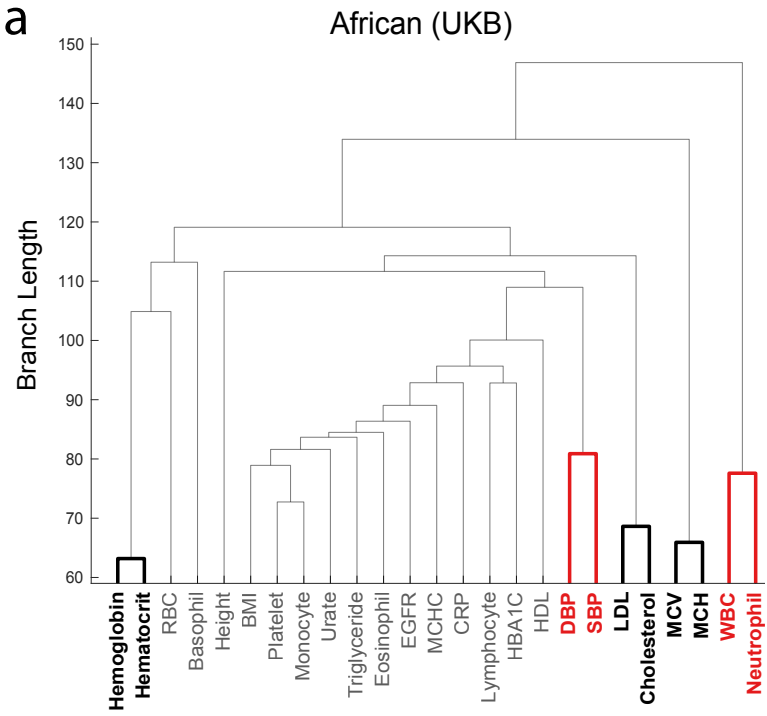

C

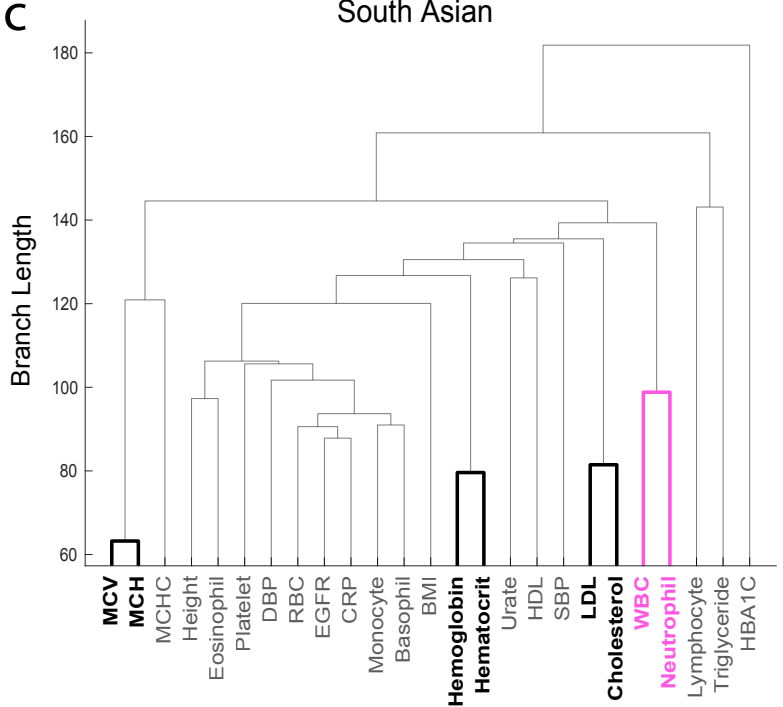

b

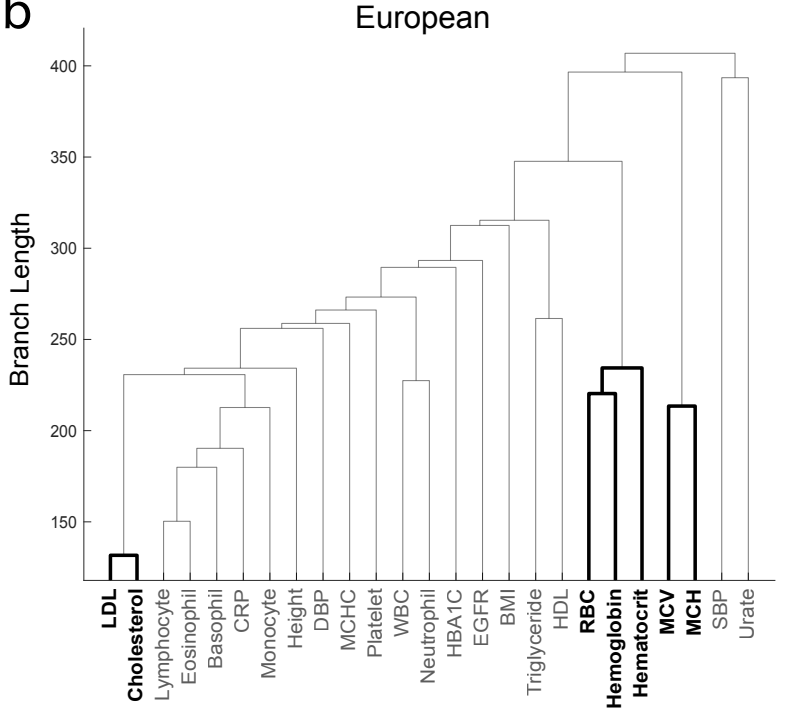

d

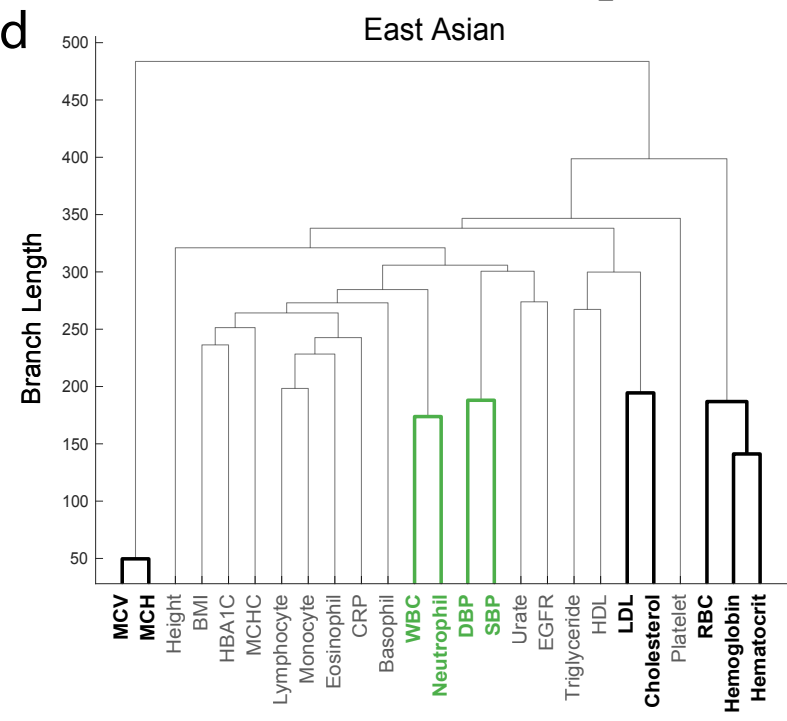

Supplementary Figure 16: Multiple prioritized trait clusters with shared core genetic trait architecture replicate in the (a) African (UKB), (b) European, (c) South Asian, and (d) East Asian ancestry cohorts. The WINGS algorithm identified prioritized phenotype clusters in each of these ancestry cohorts, denoted in each dendrogram as clades with emboldened lines. Three clusters of phenotypes were found in all ancestries (shown and labeled in black), comprising: mean corpuscular volume (MCV) and mean corpuscular hemoglobin $(\mathrm{MCH})$, hemoglobin and hematocrit, and the metabolic traits low-density lipoprotein (LDL) and cholesterol levels. In both the European and East Asian ancestry cohorts, red blood cell count (RBC) was also a member of the hemoglobin and hematocrit phenotype cluster. Two other phenotype clusters were identified in at least two ancestry cohorts. One of these clusters contains white blood cell count (WBC) and neutrophil count, and the other contains diastolic and systolic blood pressure (DBP and SBP). These two clusters are color-coded according to the ancestry cohorts in which they are prioritized. The WINGS algorithm was applied to traits from each ancestry cohort separately as described in the Online Methods section. 


\section{Supplementary Note}

\section{Standard GWA SNP-level association results for height and C- reactive protein}

In Supplementary Figure 3a and Supplementary Figure 3d, we found that, across 25 traits analyzed, height had the greatest number of genome-wide significant SNP-level associations (76,910 unique associations) in at least one ancestry. Of these SNP-level associations, 8.90\% (7,377 SNPs) replicate based off of rsID in at least two ancestry cohorts. Height is not the only trait in which the standard GWA SNP-level association test detects associations that replicate extensively across ancestries. In fact, SNP-level associations replicate in each of the 25 continuous traits that we analyze in this study.

We analyzed SNP-level associations with C-reactive protein in six ancestry cohorts: African-American (PAGE), European, South Asian, East Asian, Native Hawaiian, and Hispanic and Latin American cohorts. C-reactive protein is an example of a trait with a sparse and highly conserved genetic architecture across ancestries, as shown in Figure 2. Many SNPs within the CRP gene have been previously associated with C-reactive protein plasma levels $\sqrt{123}-125$. In our analysis, rs3091244 is genome-wide significant in only the European ancestry cohort, and has been functionally validated as influencing C-reactive protein levels 43144 . The variant rs3091244 is located in a promoter region slightly upstream of $C R P$, and it has clinical implications for both atrial fibrillation $^{[126}$ and lupus erythematosus ${ }^{127}$ (European $p=1.54 \times 10^{-116}$; East Asian $\left.p=1.15 \times 10^{-9}\right)$. For some traits, the number of SNP-level associations is small and are localized to a few genomic regions (see WBC and MCV in Supplementary Table 16.

We expanded our search for replicated GWA SNP-level association signals across ancestry cohorts by scanning for $1 \mathrm{Mb}$ regions that contained associations to the same phenotype in two or more ancestriesa process often referred to as "clumping". These windows were centered at every unique genome-wide significant SNP in any ancestry for a given trait (we refer the $1 \mathrm{Mb}$ window around the significant SNP as a "clump", Supplementary Figure 3p and Supplementary Figure 3p). In addition to the largest number of unique SNP-level associations, height also had the largest proportion of clumps containing a significant SNPlevel GWA association signal that replicated in at least two ancestry cohorts (see Supplementary Figure $3 \mathrm{~b}$ and Supplementary Figure 33). The three traits with the greatest proportion of clumps containing SNP-level GWA signals that replicate in multiple ancestry cohorts were height (77.09\% of clumps), urate (65.89\%), and low density lipoprotein $(54.40 \%)$.

In addition to the SNP-level associations on chromosome 1 surrounding the CRP gene across all six ancestry cohorts (displayed in Figure 2), there are other regions of the genome that contain significant GWA 
associations with C-reactive protein that replicate in multiple ancestry cohorts. On chromosome 2, there is a cluster of four SNPs significantly associated with C-reactive protein levels in the European, East Asian, and Hispanic and Latin American ancestry cohorts. Of these, rs1260326 (European $p=1.01 \times 10^{-55}$; East Asian $p=1.70 \times 10^{-9} ;$ Hispanic and Latin American $p=1.24 \times 10^{-20}$ ), rs780094 (European $p=9.95 \times 10^{-51}$; East Asian $p=1.70 \times 10^{-9}$; Hispanic and Latin American $p=1.14 \times 10^{-16}$ ), and rs6734238 (AfricanAmerican (PAGE) $p=3.04 \times 10^{-10}$; European $p=8.38 \times 10^{-34}$; South Asian $p=2.17 \times 10^{-9}$ ) were statistically significant in three of the six ancestry cohorts that we analyzed. Each of these three SNPs has been previously associated with C-reactive protein levels in a European ancestry cohort $128-130$. Of these three SNPs, only one (rs6734238) had previously been replicated in other ancestries (in African-American, and Hispanic and Latin American cohorts ${ }^{131}$ ).

On chromosome 19 there are 23 SNPs that are associated with CRP in the African-American PAGE, European, and Hispanic and Latin American ancestry cohorts. Two other SNPs are associated with Creactive protein in the African-American (PAGE), European, and Hispanic and Latin American cohorts, as well as the East Asian ancestry cohort. One of these two SNPs, rs7310409 (African-American (PAGE) $p=8.57 \times 10^{-9} ;$ European $p=3.57 \times 10^{-210} ;$ East Asian $p=2.72 \times 10^{-27} ;$ Hispanic and Latin American $p=$ $\left.5.35 \times 10^{-29}\right)$ located in the HNF1 homeobox A (HNF1A) gene, has been previously associated with C-reactive protein levels in only a European ancestry cohort129|130. Three additional significant SNPs in our analysis have been previously associated with European ancestry cohorts in previous studies, including: rs1169310 131 (European $p=1.52 \times 10^{-172}$; East Asian $p=1.28 \times 10^{-18}$; Hispanic and Latin American $p=1.17 \times 10^{-27}$ ), $\mathrm{rs} 1183910^{128 \mid 132}$ (European $p=5.50 \times 10^{-177}$; East Asian $p=3.16 \times 10^{-29}$; Hispanic and Latin American

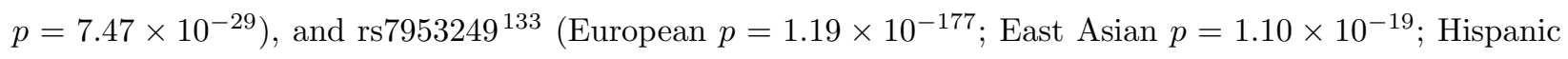
and Latin American $p=4.80 \times 10^{-29}$ ). Two SNPs, rs2259816 (European $p=2.77 \times 10^{-172}$; East Asian $p=9.33 \times 10^{-18} ;$ Hispanic and Latin American $p=1.90 \times 10^{-27}$ ) and rs7979473 (African $p=1.49 \times 10^{-9}$; East Asian $p=6.06 \times 10^{-29}$; Hispanic and Latin American $\left.p=1.56 \times 10^{-30}\right)$, have been previously associated with C-reactive protein in both African-American and Hispanic and Latin American ancestry cohorts ${ }^{131}$. There is one final group of three SNPs associated with C-reactive protein in the African-American (PAGE), European, East Asian, and Hispanic and Latin American ancestry cohorts on chromosome 19. One of them, rs4420638 (East Asian $p=9.93 \times 10^{-29}$; Hispanic and Latin American $p=2.03 \times 10^{-30}$ ), has been previously associated in a European ancestry cohort $[128|130| 132$. These four regions indicate a highly conserved SNPlevel architecture of C-reactive protein across six ancestry cohorts. Interestingly, we were unable to replicate associations with C-reactive protein across ancestries at the gene or pathway levels. 


\section{Gene and pathway association results}

Three genes, GP6, RDH13, and AGPAT5, were significantly associated with platelet count (PLC) in the African-American (PAGE) ancestry cohort and the East Asian ancestry cohort Supplementary Figure 10. Of these, no significant SNPs in the glycoprotein VI platelet (GP6) gene have been reported in the GWAS catalog for either ancestry cohort. However, a single SNP within GP6, rs1613662, has previously been associated with mean platelet volume in a GWA study analyzing a European ancestry cohort 134 . GP6 plays a critical role in platelet aggregation, and mutations have been previously associated with fetal loss $\frac{135}{13}$. The retinol dehydrogenase 13 (RDH13) gene has no reported GWAS catalog associations with platelet count, but is within $60 \mathrm{~kb}$ of a SNP significantly associated with platelet aggregation ${ }^{136}$. Of the three genes significantly associated with PLC in both the European and AIAN cohorts, 1-Acylglycerol-3-Phosphate O-Acyltransferase 5 (AGPAT5) is a member of a gene family known to play a role in immunity and inflammation response ${ }^{137}$.

Alcohol dehydrogenase 2 (ALDH2) has additionally been associated with hypertension in an elderly Japanese cohort 138 . A member of the RAS oncogene family $(R A B 8 A)$ has been shown to play a role in the inhibition of inflammatory response. In contrast, the cut like homeobox 2 CUX2 gene contains a significantly associated SNP in the array used in this study for the East Asian ancestry cohort, but it has no previous associations in a European ancestry cohort. However, CUX2 is significantly associated at the gene-level in both the European and East Asian ancestry cohorts. Although not reported as being associated with PLC in the GWAS Catalog, a single SNP, rs61745424 which encodes a missense mutation, has been previously identified as being related to the trait 139 . The gene- $\varepsilon$ association statistics for the seven genes significantly associated with PLC are available in Supplementary Table 17.

Finally, a single gene, acyl-CoA dehydrogenase family member $10(A C A D 10)$ associated in our gene-level analysis of PLC, was significant in both the European and East Asian ancestry cohorts (European gene$\varepsilon p=1.47 \times 10^{-10} ;$ East Asian gene- $\varepsilon p=2.00 \times 10^{-10}$ ) but contained no previous associations in the GWAS catalog. The African-American and Hispanic and Latin American ancestry cohorts analyzed in Qayyum et al. 140 both contain SNPs within $A C A D 10$ that are significantly associated with PLC.

In our analysis of triglyceride levels in six ancestry cohorts (African-American (PAGE), European, East Asian, South Asian, Hispanic and Latin American, and Native Hawaiian), we identified shared genetic architecture at the SNP, gene, and subnetwork level. Replicated SNPs and genes between the six ancestry cohorts are shown in Supplementary Figure 12 Supplementary Figure 13 We focus our discussion of results at the network level in the European, East Asian, and Native Hawaiian ancestry cohorts (Figure 3). In the European and East Asian ancestry cohorts, we identified 55 shared genome-wide significant associations at the gene-level. Of these results, eight genes lie in the same significantly mutated subnetwork (Hierarchical 
HotNet $p<10^{-3}$ ) when analyzing each ancestry cohort independently. Five of those eight genes belong to the apolipoprotein family of genes, including: apolipoprotein A1 (APOA1), apolipoprotein A4 (APOA4), apolipoprotein A5 (APOA5), apollipoprotein C3 (APOC3), apolipoprotein E (APOE). Specifically, the apolipoprotein play a central role in lipoprotein biosynthesis and transport. All of these genes contain SNPs previously associated with triglyceride levels in a European ancestry cohort 112117 . All five genes also contain SNPs previously associated with triglyceride levels in non-European ancestry cohorts. Specifically, $A P O A 1, A P O C 3$, and $A P O E$ each contain SNPs previously associated with triglyceride levels in AfricanAmerican and Hispanic and Latin American ancestry cohorts 1121113 . The APOA5 gene has previously been associated to triglyceride levels in an East Asian, African-American, and Hispanic and Latin American ancestry cohorts $114[118$.

The other three genes that were significantly associated with triglyceride levels in the European and East Asian ancestry cohorts are members of the largest significantly mutated subnetwork including phospholipid transfer protein (PLTP; European gene- $\varepsilon p=4.29 \times 10^{-9}$; East Asian gene- $\varepsilon p=6.66 \times 10^{-15}$ ), lipoprotein lipase $\left(L P L\right.$; European gene- $\varepsilon p=4.08 \times 10^{-13}$; East Asian gene- $\left.\varepsilon p=1.00 \times 10^{-20}\right)$, and angiopoietin like 3 (ANGPTL3; European gene- $\varepsilon p=8.86 \times 10^{-8}$; East Asian gene- $\varepsilon p=1.00 \times 10^{-20}$ ). PLTP has previously been associated with triglyceride levels in European, African-American, and Hispanic and Latin American ancestry cohorts 111 114/119 121/141. LPL is one of the most well-studied genes in the regulation of triglyceride levels. It has previously been associated with triglyceride levels in European ancestry cohorts $110-117|119-121| 141|141| 153$, East Asian ancestry cohorts $118 \mid 154$, and African ancestry cohorts as well as Hispanic and Latin American ancestry cohorts $110|112| 114|119| 152|153| 155 \mid 156$. The final gene that was genome-wide significant in both the European and East Asian ancestry cohorts, ANGPTL3, has no previous associations in the GWAS catalog and presents a novel candidate gene within the network. While not significant in any gene-level analysis, the gene ANGPTL4 (European gene- $\varepsilon p=1.00 \times 10^{-20}$; East Asian gene- $\varepsilon p=9.99 \times 10^{-1}$ ) from the same family is present in the largest subnetwork in the European cohort and also has also been previously identified as having associations in European, African, and Hispanic and Latin American ancestry cohorts $110|111| 114|152| 153 \mid 157$.

In our analysis of the European ancestry cohort from the UK Biobank, we additionally identified a set of eight genes that are connected to the core network discussed above. One of these genes is ANGPTL4, which we discussed above. Five of these genes were significant at the gene-level in the European ancestry cohort, including four apoliprotein genes (APOC1; European gene- $\varepsilon p=1.67 \times 10^{-16}$, APOC2; European gene$\varepsilon p=3.57 \times 10^{-13}, A P O C 4 ;$ European gene- $\varepsilon p=3.72 \times 10^{-13}$, and $A P O B ;$ European gene- $\varepsilon p=1.00 \times 10^{-20}$ ) and lipase maturation factor $1\left(L M F 1\right.$; European gene- $\left.\varepsilon p=8.03 \times 10^{-7}\right)$. Each of these genes have been previously associated with triglyceride levels in a European ancestry cohort 114 . Additional associations 
were also found in that same study which conducted a meta-analysis of European, African-American, and Hispanic and Latin American ancestry cohorts. The final two genes included in the significantly mutated subnetwork of the European ancestral cohort, APOL1 and HBA1, were not were not identified as genomewide significant by gene- $\varepsilon$ and have no previous SNP-level associations with triglyceride levels in the GWAS Catalog. Interestingly, both APOL1 (Native Hawaiian gene- $\varepsilon p=8.89 \times 10^{-11}$ ) and $H B A 1$ (Native Hawaiian gene- $\varepsilon p=2.46 \times 10^{-10}$ ) were both identified as genome-wide significant by gene- $\varepsilon$ in our analysis of the Native Hawaiian ancestry cohort and the interaction between them was identified in our Hierarchical HotNet analysis as present in both the European and Native Hawaiian ancestry cohorts.

In addition to APOL1 and $H B A 1$, six more genes are connected to the core network of genes that overlap in the East Asian and European significantly mutated subnetworks. Of these, both HBA2 and B4GALT3 are significant at the gene-level in the Native Hawaiian ancestry cohort alone. They are each connected to genes identified in both the European and Native Hawaiian ancestry cohorts as members of the largest significantly mutated subnetwork. The final three genes include kallikrein related peptidase 8 (KLK8), pancreatic lipase PNLIP, and wnt family member 4 (WNT4) which were not significant at the gene-level and did not contain previous SNP-level associations in the GWAS catalog.

In the largest significantly mutated subnetwork identified in our analysis of the East Asian ancestry cohort, we identified seven genes that were not shared by the networks in other ancestry cohorts. One of these genes, beta-secretase 1 ( $B A C E 1$; East Asian gene- $\varepsilon p=3.57 \times 10^{-13}$; European gene- $\varepsilon p=5.55 \times 10^{-} 17$ ), was significant at the gene-level but contained no previously associated SNPs in any cohort in the GWAS catalog. BACE1 plays a role in the metabolism of amyloid beta precursor protein 158 . Three of the genes within the network identified in the East Asian ancestry cohort contain previously associated SNPs in both European and non-European ancestry cohorts, including: cholesteryl ester transfer protein $(C E T P)$, proprotein convertase subtilisin/kexin type 6 (PCSK6), and proprotein convertase subtilisin/kexin type 7 (PCSK 7$)^{112[119}$. The final three genes in the significantly mutated subnetwork identified in the East Asian ancestry cohort were not significant at the gene-level and do not contain previously associated SNPs in the GWAS catalog in any ancestral cohort. Lecithin-cholesterol acyltransferase $(L C A T)$ is involved in cholesterol biosynthesis and apolipoprotein $\mathrm{F}(A P O F)$ encodes one of the minor apolipoprotein genes present in plasma. Finally, tyrosine-protein kinase receptor 3 (TYRO3) plays a role in ligand recognition and cell metabolism $\frac{159}{}$. The gene- $\varepsilon$ p-values in each ancestry cohort for each of the 28 genes discussed here are shown in Supplementary Table 18 . 


\section{WHI study cohort description}

The Women's Health Initiative (WHI) is a long-term, prospective, multi-center cohort study investigating post-menopausal women's health in the US. WHI was funded by the National Institutes of Health and the National Heart, Lung, and Blood Institute to study strategies to prevent heart disease, breast 124 cancer, colon cancer, and osteoporotic fractures in women 50-79 years of age. WHI involves 161,808 women recruited between 1993 and 1998 at 40 centers across the US. The study consists of two parts: the WHI Clinical Trial which was a randomized clinical trial of hormone therapy, dietary modification, and calcium/Vitamin D supplementation, and the WHI Observational Study, which focused on many of the inequities in women's health research and provided practical information about incidence, risk factors, and interventions related to heart disease, cancer, and osteoporotic fractures. For this project, women who self identified as European were excluded from the study sample (dbGaP study accession number: phs000227).

\section{HCHC/SOL study cohort description}

The Hispanic Community Health Study / Study of Latinos (HCHS/SOL) is a multi center study of Hispanic/Latino populations with the goal of determining the role of acculturation in the prevalence and development of diseases, and to identify other traits that impact Hispanic/Latino health. The study is sponsored by the National Heart, Lung, and Blood Institute (NHLBI) and other institutes, centers, and offices of the National Institutes of Health (NIH). Recruitment began in 2006 with a target population of 16,000 persons of Cuban, Puerto Rican, Dominican, Mexican or Central/South American origin. Household sampling was employed as part of the study design. Participants were recruited through four sites affiliated with San Diego State University, Northwestern University in Chicago, Albert Einstein College of Medicine in Bronx, New York, and the University of Miami. Researchers from seven academic centers provided scientific and logistical support. Study participants who were self-identified Hispanic/Latino and aged 18-74 years underwent extensive psycho-social and clinical assessments during 2008-2011. A re-examination of the HCHS/SOL cohort is conducted during 2015-2017. Annual telephone follow-up interviews are ongoing since study inception to determine health outcomes of interest. (dbGaP study accession number: phs000555).

\section{BioMe Biobank study cohort description}

The Charles Bronfman Institute for Personalized Medicine at Mount Sinai Medical Center (MSMC), BioMeTM 54 BioBank (BioMe) is an EMR-linked bio-repository drawing from Mount Sinai Medical Center consented patients which were drawn from a population of over 70,000 inpatients and 800,000 outpatients annually. 
The MSMC serves diverse local communities of upper Manhattan, including Central Harlem (86\% African American), East Harlem (88\% Hispanic/Latino), and Upper East Side (88\% Caucasian/White) with broad health disparities. BioMeTM 58 enrolled over 26,500 participants from September 2007 through August 2013, with 25\% African American, 36\% Hispanic/Latino (primarily of Caribbean origin), 30\% Caucasian, and $9 \%$ of Other ancestry. The BioMeTM 60 population reflects community-level disease burdens and health disparities with broad public health impact. Biobank operations are fully integrated in clinical care processes, including direct recruitment from clinical sites waiting areas and phlebotomy stations by dedicate Biobank recruiters independent of clinical care providers, prior to or following a clinician standard of care visit. Recruitment currently occurs at a broad spectrum of over 30 clinical care sites. Study participants of self-reported European ancestry were not included in this analysis. (dbGaP study accession number: phs000925).

\section{MEC study cohort description}

The Multiethnic Cohort (MEC) is a population-based prospective cohort study including approximately 215,000 men and women from Hawaii and California. All participants were 45-75 years of age at baseline, and primarily of 5 ancestries: Japanese Americans, African Americans, European Americans, Hispanic/Latinos, and Native Hawaiians. MEC was funded by the National Cancer Institute in 1993 to examine lifestyle risk factors and genetic susceptibility to cancer. All eligible cohort members completed baseline and follow-up questionnaires. Within the PAGE II investigation, MEC proposes to study: 1) diseases for which we have DNA available for large numbers of cases and controls (breast, prostate, and colorectal cancer, diabetes, and obesity); 2) common traits that are risk factors for these diseases (e.g., body mass index / weight, waist-to-hip ratio, height), and 3) relevant disease-associated biomarkers (e.g., fasting insulin and lipids, steroid hormones). The specific aims are: 1) to determine the population-based epidemiologic profile (allele frequency, main effect, heterogeneity by disease characteristics) of putative causal variants in the five racial/ethnic groups in MEC; 2) for variants displaying effect heterogeneity across ethnic/racial groups, we will utilize differences in LD to identify a more complete spectrum of associated variants at these loci; 3 ) investigate gene $\mathrm{x}$ gene and gene $\mathrm{x}$ environment interactions to identify modifiers; 4) examine the associations of putative causal variants with already measured intermediate phenotypes (e.g., plasma insulin, lipids, steroid hormones); and 5) for variants that do not fall within known genes, start to investigate their relationships with gene expression and epigenetic patterns in small genomic studies. For this project, MEC contributed African American, Japanese American, and Native Hawaiian samples.(dbGaP study accession number: phs000220). 


\section{References}

[1] Akiko Nagai, Makoto Hirata, Yoichiro Kamatani, Kaori Muto, Koichi Matsuda, Yutaka Kiyohara, Toshiharu Ninomiya, Akiko Tamakoshi, Zentaro Yamagata, Taisei Mushiroda, et al. Overview of the biobank japan project: study design and profile. Journal of epidemiology, 27(Supplement_III):S2-S8, 2017.

[2] Alicia R Martin, Christopher R Gignoux, Raymond K Walters, Genevieve L Wojcik, Benjamin M Neale, Simon Gravel, Mark J Daly, Carlos D Bustamante, and Eimear E Kenny. Human demographic history impacts genetic risk prediction across diverse populations. The American Journal of Human Genetics, 100(4):635-649, 2017.

[3] Pamela L Sankar and Lisa S Parker. The precision medicine initiative's all of us research program: an agenda for research on its ethical, legal, and social issues. Genetics in Medicine, 19(7):743-750, 2017.

[4] Clare Bycroft, Colin Freeman, Desislava Petkova, Gavin Band, Lloyd T Elliott, Kevin Sharp, Allan Motyer, Damjan Vukcevic, Olivier Delaneau, Jared O'Connell, et al. The uk biobank resource with deep phenotyping and genomic data. Nature, 562(7726):203-209, 2018.

[5] Alicia R Martin, Masahiro Kanai, Yoichiro Kamatani, Yukinori Okada, Benjamin M Neale, and Mark J Daly. Clinical use of current polygenic risk scores may exacerbate health disparities. Nature genetics, $51(4): 584,2019$.

[6] Genevieve L Wojcik, Mariaelisa Graff, Katherine K Nishimura, Ran Tao, Jeffrey Haessler, Christopher R Gignoux, Heather M Highland, Yesha M Patel, Elena P Sorokin, Christy L Avery, et al. Genetic analyses of diverse populations improves discovery for complex traits. Nature, 570(7762):514-518, 2019.

[7] Mashaal Sohail, Robert M Maier, Andrea Ganna, Alex Bloemendal, Alicia R Martin, Michael C Turchin, Charleston WK Chiang, Joel Hirschhorn, Mark J Daly, Nick Patterson, et al. Polygenic adaptation on height is overestimated due to uncorrected stratification in genome-wide association studies. Elife, 8:e39702, 2019.

[8] Jeremy J Berg, Arbel Harpak, Nasa Sinnott-Armstrong, Anja Moltke Joergensen, Hakhamanesh Mostafavi, Yair Field, Evan August Boyle, Xinjun Zhang, Fernando Racimo, Jonathan K Pritchard, et al. Reduced signal for polygenic adaptation of height in uk biobank. Elife, 8:e39725, 2019.

[9] Pak C Sham and Shaun M Purcell. Statistical power and significance testing in large-scale genetic studies. Nature Reviews Genetics, 15(5):335-346, 2014. 
[10] Alkes L Price, Chris CA Spencer, and Peter Donnelly. Progress and promise in understanding the genetic basis of common diseases. Proceedings of the Royal Society B: Biological Sciences, 282(1821): $20151684,2015$.

[11] Peter M Visscher, Naomi R Wray, Qian Zhang, Pamela Sklar, Mark I McCarthy, Matthew A Brown, and Jian Yang. 10 years of gwas discovery: biology, function, and translation. The American Journal of Human Genetics, 101(1):5-22, 2017.

[12] Jonathan K Pritchard and Molly Przeworski. Linkage disequilibrium in humans: models and data. The American Journal of Human Genetics, 69(1):1-14, 2001.

[13] Jeremy J Berg and Graham Coop. A population genetic signal of polygenic adaptation. PLoS Genet, 10(8):e1004412, 2014.

[14] Mattias Jakobsson, Michael D Edge, and Noah A Rosenberg. The relationship between fst and the frequency of the most frequent allele. Genetics, 193(2):515-528, 2013.

[15] Michael D Edge and Noah A Rosenberg. Upper bounds on fst in terms of the frequency of the most frequent allele and total homozygosity: the case of a specified number of alleles. Theoretical population biology, 97:20-34, 2014.

[16] Michael D Edge and Noah A Rosenberg. A general model of the relationship between the apportionment of human genetic diversity and the apportionment of human phenotypic diversity. Human biology, 87 (4):313-337, 2015.

[17] Farhad Hormozdiari, Anthony Zhu, Gleb Kichaev, Chelsea J-T Ju, Ayellet V Segrè, Jong Wha J Joo, Hyejung Won, Sriram Sankararaman, Bogdan Pasaniuc, Sagiv Shifman, et al. Widespread allelic heterogeneity in complex traits. The American Journal of Human Genetics, 100(5):789-802, 2017.

[18] John Novembre and Nicholas H Barton. Tread lightly interpreting polygenic tests of selection. Genetics, 208(4):1351, 2018.

[19] Noah A Rosenberg, Michael D Edge, Jonathan K Pritchard, and Marcus W Feldman. Interpreting polygenic scores, polygenic adaptation, and human phenotypic differences. Evolution, medicine, and public health, 2019(1):26-34, 2019.

[20] Arbel Harpak and Molly Przeworski. The evolution of group differences in changing environments. PLoS Biology, 19(1):e3001072, 2021. 
[21] Luisa Pereira, Leon Mutesa, Paulina Tindana, and Michèle Ramsay. African genetic diversity and adaptation inform a precision medicine agenda. Nature Reviews Genetics, 2021.

[22] Arun Durvasula and Kirk E Lohmueller. Negative selection on complex traits limits phenotype prediction accuracy between populations. The American Journal of Human Genetics, 2021.

[23] Chief Ben-Eghan, Rosie Sun, Jose Sergio Hleap, Alex Diaz-Papkovich, Hans Markus Munter, Audrey V Grant, Charles Dupras, and Simon Gravel. Don't ignore genetic data from minority populations, 2020.

[24] Nasa Sinnott-Armstrong, Yosuke Tanigawa, David Amar, Nina J Mars, Matthew Aguirre, Guhan Ram Venkataraman, Michael Wainberg, Hanna M Ollila, James P Pirruccello, Junyang Qian, et al. Genetics of 38 blood and urine biomarkers in the uk biobank. BioRxiv, page 660506, 2019.

[25] Alice B Popejoy and Stephanie M Fullerton. Genomics is failing on diversity. Nature News, 538(7624): $161,2016$.

[26] Carlos D Bustamante, M Francisco, and Esteban G Burchard. Genomics for the world. Nature, 475 (7355):163-165, 2011.

[27] Valentin Hivert, Julia Sidorenko, Florian Rohart, Michael E Goddard, Jian Yang, Naomi R Wray, Loic Yengo, and Peter M Visscher. Estimation of non-additive genetic variance in human complex traits from a large sample of unrelated individuals. bioRxiv, 2020.

[28] Farhad Hormozdiari, Emrah Kostem, Eun Yong Kang, Bogdan Pasaniuc, and Eleazar Eskin. Identifying causal variants at loci with multiple signals of association. Genetics, 198(2):497-508, 2014.

[29] Farhad Hormozdiari, Martijn Van De Bunt, Ayellet V Segre, Xiao Li, Jong Wha J Joo, Michael Bilow, Jae Hoon Sul, Sriram Sankararaman, Bogdan Pasaniuc, and Eleazar Eskin. Colocalization of gwas and eqtl signals detects target genes. The American Journal of Human Genetics, 99(6):1245-1260, 2016.

[30] Nathan LaPierre, Kodi Taraszka, Helen Huang, Rosemary He, Farhad Hormozdiari, and Eleazar Eskin. Identifying causal variants by fine mapping across multiple studies. In International Conference on Research in Computational Molecular Biology, pages 257-258. Springer, 2020.

[31] Lorin Crawford, Ping Zeng, Sayan Mukherjee, and Xiang Zhou. Detecting epistasis with the marginal epistasis test in genetic mapping studies of quantitative traits. PLoS genetics, 13(7):e1006869, 2017.

[32] Michael C Turchin and Matthew Stephens. Bayesian multivariate reanalysis of large genetic studies identifies many new associations. PLoS genetics, 15(10):e1008431, 2019. 
[33] Xiang Zhou and Matthew Stephens. Genome-wide efficient mixed-model analysis for association studies. Nature genetics, 44(7):821-824, 2012.

[34] Matthew Stephens. A unified framework for association analysis with multiple related phenotypes. PloS one, 8(7):e65245, 2013.

[35] Sarah M Urbut, Gao Wang, Peter Carbonetto, and Matthew Stephens. Flexible statistical methods for estimating and testing effects in genomic studies with multiple conditions. Nature genetics, $51(1)$ : 187-195, 2019.

[36] Brielin C Brown, Chun Jimmie Ye, Alkes L Price, Noah Zaitlen, Asian Genetic Epidemiology Network Type 2 Diabetes Consortium, et al. Transethnic genetic-correlation estimates from summary statistics. The American Journal of Human Genetics, 99(1):76-88, 2016.

[37] Kevin J Galinsky, Yakir A Reshef, Hilary K Finucane, Po-Ru Loh, Noah Zaitlen, Nick J Patterson, Brielin C Brown, and Alkes L Price. Estimating cross-population genetic correlations of causal effect sizes. Genetic epidemiology, 43(2):180-188, 2019.

[38] Christopher S Carlson, Tara C Matise, Kari E North, Christopher A Haiman, Megan D Fesinmeyer, Steven Buyske, Fredrick R Schumacher, Ulrike Peters, Nora Franceschini, Marylyn D Ritchie, et al. Generalization and dilution of association results from european gwas in populations of non-european ancestry: the page study. PLoS Biol, 11(9):e1001661, 2013.

[39] Jimmy Z Liu, Suzanne Van Sommeren, Hailiang Huang, Siew C Ng, Rudi Alberts, Atsushi Takahashi, Stephan Ripke, James C Lee, Luke Jostins, Tejas Shah, et al. Association analyses identify 38 susceptibility loci for inflammatory bowel disease and highlight shared genetic risk across populations. Nature genetics, 47(9):979, 2015.

[40] Adam Eyre-Walker. Genetic architecture of a complex trait and its implications for fitness and genomewide association studies. Proceedings of the National Academy of Sciences, 107(suppl 1):1752-1756, 2010.

[41] Huwenbo Shi, Kathryn S Burch, Ruth Johnson, Malika K Freund, Gleb Kichaev, Nicholas Mancuso, Astrid M Manuel, Natalie Dong, and Bogdan Pasaniuc. Localizing components of shared transethnic genetic architecture of complex traits from gwas summary data. The American Journal of Human Genetics, 2020.

[42] Huwenbo Shi, Steven Gazal, Masahiro Kanai, Evan M Koch, Armin P Schoech, Katherine M Siewert, Samuel S Kim, Yang Luo, Tiffany Amariuta, Hailiang Huang, et al. Population-specific causal disease 
effect sizes in functionally important regions impacted by selection. Nature Communications, 12(1): $1-15,2021$.

[43] AJ Szalai, J Wu, EM Lange, MA McCrory, CD Langefeld, A Williams, SO Zakharkin, Varghese George, DB Allison, GS Cooper, et al. Single-nucleotide polymorphisms in the c-reactive protein (crp) gene promoter that affect transcription factor binding, alter transcriptional activity, and associate with differences in baseline serum crp level. Journal of Molecular Medicine, 83(6):440-447, 2005.

[44] Shi-Chao Zhang, Ming-Yu Wang, Jun-Rui Feng, Yue Chang, Shang-Rong Ji, and Yi Wu. Reversible promoter methylation determines fluctuating expression of acute phase proteins. Elife, 9:e51317, 2020.

[45] Alicia R Martin, Meng Lin, Julie M Granka, Justin W Myrick, Xiaomin Liu, Alexandra Sockell, Elizabeth G Atkinson, Cedric J Werely, Marlo Möller, Manjinder S Sandhu, et al. An unexpectedly complex architecture for skin pigmentation in africans. Cell, 171(6):1340-1353, 2017.

[46] Gao Wang, Abhishek Sarkar, Peter Carbonetto, and Matthew Stephens. A simple new approach to variable selection in regression, with application to genetic fine mapping. Journal of the Royal Statistical Society: Series B (Statistical Methodology), 82(5):1273-1300, 2020.

[47] Brian L Browning and Sharon R Browning. Efficient multilocus association testing for whole genome association studies using localized haplotype clustering. Genetic Epidemiology: The Official Publication of the International Genetic Epidemiology Society, 31(5):365-375, 2007.

[48] Michael C Wu, Seunggeun Lee, Tianxi Cai, Yun Li, Michael Boehnke, and Xihong Lin. Rare-variant association testing for sequencing data with the sequence kernel association test. The American Journal of Human Genetics, 89(1):82-93, 2011.

[49] Jimmy Z Liu, Allan F Mcrae, Dale R Nyholt, Sarah E Medland, Naomi R Wray, Kevin M Brown, Nicholas K Hayward, Grant W Montgomery, Peter M Visscher, Nicholas G Martin, et al. A versatile gene-based test for genome-wide association studies. The American Journal of Human Genetics, 87 (1):139-145, 2010.

[50] Christiaan A de Leeuw, Joris M Mooij, Tom Heskes, and Danielle Posthuma. Magma: generalized gene-set analysis of gwas data. PLoS Comput Biol, 11(4):e1004219, 2015.

[51] Qian S Zhang, Brian L Browning, and Sharon R Browning. Genome-wide haplotypic testing in a finnish cohort identifies a novel association with low-density lipoprotein cholesterol. European Journal of Human Genetics, 23(5):672-677, 2015. 
[52] Xiang Zhu and Matthew Stephens. Bayesian large-scale multiple regression with summary statistics from genome-wide association studies. The annals of applied statistics, 11(3):1561, 2017.

[53] Wei Cheng, Sohini Ramachandran, and Lorin Crawford. Estimation of non-null snp effect size distributions enables the detection of enriched genes underlying complex traits. bioRxiv, page 597484, 2020.

[54] John D Storey and Robert Tibshirani. Statistical significance for genomewide studies. Proceedings of the National Academy of Sciences, 100(16):9440-9445, 2003.

[55] Matthew Stephens. False discovery rates: a new deal. Biostatistics, 18(2):275-294, 2017.

[56] Priyanka Nakka, Benjamin J Raphael, and Sohini Ramachandran. Gene and network analysis of common variants reveals novel associations in multiple complex diseases. Genetics, 204(2):783-798, 2016.

[57] Benjamin M Neale, Sarah E Medland, Stephan Ripke, Philip Asherson, Barbara Franke, Klaus-Peter Lesch, Stephen V Faraone, Thuy Trang Nguyen, Helmut Schäfer, Peter Holmans, et al. Meta-analysis of genome-wide association studies of attention-deficit/hyperactivity disorder. Journal of the American Academy of Child \& Adolescent Psychiatry, 49(9):884-897, 2010.

[58] Ditte Demontis, Raymond K Walters, Joanna Martin, Manuel Mattheisen, Thomas D Als, Esben Agerbo, Gísli Baldursson, Rich Belliveau, Jonas Bybjerg-Grauholm, Marie Bækvad-Hansen, et al. Discovery of the first genome-wide significant risk loci for attention deficit/hyperactivity disorder. Nature genetics, 51(1):63-75, 2019.

[59] Mark D Leiserson, Fabio Vandin, Hsin-Ta Wu, Jason R Dobson, and Benjamin R Raphael. Pan-cancer identification of mutated pathways and protein complexes, 2014.

[60] Lenore Cowen, Trey Ideker, Benjamin J Raphael, and Roded Sharan. Network propagation: a universal amplifier of genetic associations. Nature Reviews Genetics, 18(9):551, 2017.

[61] Matthew A Reyna, Mark DM Leiserson, and Benjamin J Raphael. Hierarchical hotnet: identifying hierarchies of altered subnetworks. Bioinformatics, 34(17):i972-i980, 2018.

[62] Noah Zaitlen and Peter Kraft. Heritability in the genome-wide association era. Human genetics, 131 (10):1655-1664, 2012. 
[63] Brendan K Bulik-Sullivan, Po-Ru Loh, Hilary K Finucane, Stephan Ripke, Jian Yang, Nick Patterson, Mark J Daly, Alkes L Price, and Benjamin M Neale. Ld score regression distinguishes confounding from polygenicity in genome-wide association studies. Nature genetics, 47(3):291-295, 2015.

[64] Jian Yang, Andrew Bakshi, Zhihong Zhu, Gibran Hemani, Anna AE Vinkhuyzen, Sang Hong Lee, Matthew R Robinson, John RB Perry, Ilja M Nolte, Jana V van Vliet-Ostaptchouk, et al. Genetic variance estimation with imputed variants finds negligible missing heritability for human height and body mass index. Nature genetics, 47(10):1114, 2015.

[65] Xuan Zhou, Hae Kyung Im, and S Hong Lee. Core greml for estimating covariance between random effects in linear mixed models for complex trait analyses. Nature communications, 11(1):1-11, 2020.

[66] Huwenbo Shi, Gleb Kichaev, and Bogdan Pasaniuc. Contrasting the genetic architecture of 30 complex traits from summary association data. The American Journal of Human Genetics, 99(1):139-153, 2016.

[67] Nasa Sinnott-Armstrong, Sahin Naqvi, Manuel A Rivas, and Jonathan K Pritchard. Gwas of three molecular traits highlights core genes and pathways alongside a highly polygenic background. eLife, 2021.

[68] Tomaz Berisa and Joseph K Pickrell. Approximately independent linkage disequilibrium blocks in human populations. Bioinformatics, 32(2):283, 2016.

[69] Xue Sun, Feng Jiang, Rong Zhang, Shan-shan Tang, Miao Chen, Dan-feng Peng, Jing Yan, Tao Wang, Shi-yun Wang, Yu-qian Bao, et al. Serum uric acid levels are associated with polymorphisms in the slc2a9, sf1, and gckr genes in a chinese population. Acta Pharmacologica Sinica, 35(11):1421-1427, 2014.

[70] Anna Köttgen, Eva Albrecht, Alexander Teumer, Veronique Vitart, Jan Krumsiek, Claudia Hundertmark, Giorgio Pistis, Daniela Ruggiero, Conall M O'Seaghdha, Toomas Haller, et al. Genome-wide association analyses identify 18 new loci associated with serum urate concentrations. Nature genetics, 45(2):145-154, 2013.

[71] AJ Phipps-Green, ME Merriman, R Topless, S Altaf, GW Montgomery, C Franklin, GT Jones, AM Van Rij, Douglas White, LK Stamp, et al. Twenty-eight loci that influence serum urate levels: analysis of association with gout. Annals of the rheumatic diseases, 75(1):124-130, 2016.

[72] Jade E Hollis-Moffatt, Amanda J Phipps-Green, Brett Chapman, Gregory T Jones, Andre van Rij, Peter J Gow, Andrew A Harrison, John Highton, Peter B Jones, Grant W Montgomery, et al. The 
renal urate transporter slc17a1 locus: confirmation of association with gout. Arthritis research \& therapy, 14(2):R92, 2012.

[73] Akiyoshi Nakayama, Hirotaka Matsuo, Takuya Shimizu, Hiraku Ogata, Yuzo Takada, Hiroshi Nakashima, Takahiro Nakamura, Seiko Shimizu, Toshinori Chiba, Masayuki Sakiyama, et al. A common missense variant of monocarboxylate transporter 9 (mct9/slc16a9) gene is associated with renal overload gout, but not with all gout susceptibility. Human cell, 26(4):133-136, 2013.

[74] Leo Breiman et al. Statistical modeling: The two cultures (with comments and a rejoinder by the author). Statistical science, 16(3):199-231, 2001.

[75] Galit Shmueli et al. To explain or to predict? Statistical science, 25(3):289-310, 2010.

[76] Xiang Zhou, Peter Carbonetto, and Matthew Stephens. Polygenic modeling with bayesian sparse linear mixed models. PLoS Genet, 9(2):e1003264, 2013.

[77] Lucia A Hindorff, Vence L Bonham, Lawrence C Brody, Margaret EC Ginoza, Carolyn M Hutter, Teri A Manolio, and Eric D Green. Prioritizing diversity in human genomics research. Nature Reviews Genetics, 19(3):175, 2018.

[78] Roseann E Peterson, Karoline Kuchenbaecker, Raymond K Walters, Chia-Yen Chen, Alice B Popejoy, Sathish Periyasamy, Max Lam, Conrad Iyegbe, Rona J Strawbridge, Leslie Brick, et al. Genome-wide association studies in ancestrally diverse populations: opportunities, methods, pitfalls, and recommendations. Cell, 179(3):589-603, 2019.

[79] Jon McClellan and Mary-Claire King. Genetic heterogeneity in human disease. Cell, 141(2):210-217, 2010.

[80] Evan A Boyle, Yang I Li, and Jonathan K Pritchard. An expanded view of complex traits: from polygenic to omnigenic. Cell, 169(7):1177-1186, 2017.

[81] Laura K Hayward and Guy Sella. Polygenic adaptation after a sudden change in environment. BioRxiv, page $792952,2019$.

[82] Barbara Domingues Bitarello and Iain Mathieson. Polygenic scores for height in admixed populations. bioRxiv, 2020.

[83] Davide Marnetto, Katri Pärna, Kristi Läll, Ludovica Molinaro, Francesco Montinaro, Toomas Haller, Mait Metspalu, Reedik Mägi, Krista Fischer, and Luca Pagani. Ancestry deconvolution and par- 
tial polygenic score can improve susceptibility predictions in recently admixed individuals. Nature communications, 11(1):1-9, 2020.

$[84]$

[85] Cristen J Willer, Yun Li, and Gonçalo R Abecasis. Metal: fast and efficient meta-analysis of genomewide association scans. Bioinformatics, 26(17):2190-2191, 2010.

[86] Dan-Yu Lin, Ran Tao, William D Kalsbeek, Donglin Zeng, Franklyn Gonzalez II, Lindsay FernándezRhodes, Mariaelisa Graff, Gary G Koch, Kari E North, and Gerardo Heiss. Genetic association analysis under complex survey sampling: the hispanic community health study/study of latinos. The American Journal of Human Genetics, 95(6):675-688, 2014.

[87] Jian Yang, Noah A Zaitlen, Michael E Goddard, Peter M Visscher, and Alkes L Price. Advantages and pitfalls in the application of mixed-model association methods. Nature genetics, 46(2):100-106, 2014.

[88] Scott I Vrieze, William G Iacono, and Matt McGue. Confluence of genes, environment, development, and behavior in a post-gwas world. Development and Psychopathology, 24(4):1195, 2012.

[89] Suzanne H Gage, George Davey Smith, Jennifer J Ware, Jonathan Flint, and Marcus R Munafo. G= e: What gwas can tell us about the environment. PLoS Genetics, 12(2):e1005765, 2016.

[90] Luisa N. Borrell, Jennifer R. Elhawary, Elena Fuentes-Afflick, Jonathan Witonsky, Nirav Bhakta, Alan H.B. Wu, Kirsten Bibbins-Domingo, Jos Ã (c) R. RodrÃguez-Santana, Michael A. Lenoir, James R. Gavin, Rick A. Kittles, Noah A. Zaitlen, David S. Wilkes, Neil R. Powe, Elad Ziv, and Esteban G. Burchard. Race and genetic ancestry in medicine â a time for reckoning with racism. New England Journal of Medicine, 0(0):null, 0. doi: 10.1056/NEJMms2029562. URL https://doi.org/10.1056/ NEJMms2029562,

[91] Yan Zhang, Guanghao Qi, Ju-Hyun Park, and Nilanjan Chatterjee. Estimation of complex effect-size distributions using summary-level statistics from genome-wide association studies across 32 complex traits. Nature genetics, 50(9):1318-1326, 2018.

[92] Alexander I Young, Stefania Benonisdottir, Molly Przeworski, and Augustine Kong. Deconstructing the sources of genotype-phenotype associations in humans. Science, 365(6460):1396-1400, 2019.

[93] Farid Rajabli, Briseida E Feliciano, Katrina Celis, Kara L Hamilton-Nelson, Patrice L Whitehead, Larry D Adams, Parker L Bussies, Clara P Manrique, Alejandra Rodriguez, Vanessa Rodriguez, et al. 
Ancestral origin of apoe $\varepsilon 4$ alzheimer disease risk in puerto rican and african american populations. PLoS genetics, 14(12), 2018.

[94] Antonio Fabregat, Konstantinos Sidiropoulos, Phani Garapati, Marc Gillespie, Kerstin Hausmann, Robin Haw, Bijay Jassal, Steven Jupe, Florian Korninger, Sheldon McKay, et al. The reactome pathway knowledgebase. Nucleic acids research, 44(D1):D481-D487, 2016.

[95] Sabry Razick, George Magklaras, and Ian M Donaldson. irefindex: a consolidated protein interaction database with provenance. BMC bioinformatics, 9(1):405, 2008.

[96] David H Alexander, John Novembre, and Kenneth Lange. Fast model-based estimation of ancestry in unrelated individuals. Genome research, 19(9):1655-1664, 2009.

[97] Christopher C Chang, Carson C Chow, Laurent CAM Tellier, Shashaank Vattikuti, Shaun M Purcell, and James J Lee. Second-generation plink: rising to the challenge of larger and richer datasets. Gigascience, 4(1):s13742-015, 2015.

[98] Masato Akiyama, Yukinori Okada, Masahiro Kanai, Atsushi Takahashi, Yukihide Momozawa, Masashi Ikeda, Nakao Iwata, Shiro Ikegawa, Makoto Hirata, Koichi Matsuda, et al. Genome-wide association study identifies 112 new loci for body mass index in the japanese population. Nature genetics, 49(10): $1458,2017$.

[99] Masahiro Kanai, Masato Akiyama, Atsushi Takahashi, Nana Matoba, Yukihide Momozawa, Masashi Ikeda, Nakao Iwata, Shiro Ikegawa, Makoto Hirata, Koichi Matsuda, et al. Genetic analysis of quantitative traits in the japanese population links cell types to complex human diseases. Nature genetics, 50(3):390-400, 2018.

[100] Masato Akiyama, Kazuyoshi Ishigaki, Saori Sakaue, Yukihide Momozawa, Momoko Horikoshi, Makoto Hirata, Koichi Matsuda, Shiro Ikegawa, Atsushi Takahashi, Masahiro Kanai, et al. Characterizing rare and low-frequency height-associated variants in the japanese population. Nature communications, 10 (1):1-11, 2019.

[101] Peter H Sudmant, Tobias Rausch, Eugene J Gardner, Robert E Handsaker, Alexej Abyzov, John Huddleston, Yan Zhang, Kai Ye, Goo Jun, Markus Hsi-Yang Fritz, et al. An integrated map of structural variation in 2,504 human genomes. Nature, 526(7571):75-81, 2015.

[102] Anders Bergström, Shane A McCarthy, Ruoyun Hui, Mohamed A Almarri, Qasim Ayub, Petr Danecek, Yuan Chen, Sabine Felkel, Pille Hallast, Jack Kamm, et al. Insights into human genetic variation and population history from 929 diverse genomes. Science, 367(6484), 2020. 
[103] Gad Abraham, Yixuan Qiu, and Michael Inouye. Flashpca2: principal component analysis of biobankscale genotype datasets. Bioinformatics, 2017.

[104] Alexander Gusev, Arthur Ko, Huwenbo Shi, Gaurav Bhatia, Wonil Chung, Brenda WJH Penninx, Rick Jansen, Eco JC De Geus, Dorret I Boomsma, Fred A Wright, et al. Integrative approaches for large-scale transcriptome-wide association studies. Nature genetics, 48(3):245, 2016.

[105] Priyanka Nakka, Natalie P Archer, Heng Xu, Philip J Lupo, Benjamin J Raphael, Jun J Yang, and Sohini Ramachandran. Novel gene and network associations found for acute lymphoblastic leukemia using case-control and family-based studies in multiethnic populations. Cancer Epidemiology and Prevention Biomarkers, 26(10):1531-1539, 2017.

[106] Jishnu Das and Haiyuan Yu. Hint: High-quality protein interactomes and their applications in understanding human disease. BMC systems biology, 6(1):92, 2012.

[107] Thomas Rolland, Murat Taşan, Benoit Charloteaux, Samuel J Pevzner, Quan Zhong, Nidhi Sahni, Song Yi, Irma Lemmens, Celia Fontanillo, Roberto Mosca, et al. A proteome-scale map of the human interactome network. Cell, 159(5):1212-1226, 2014.

[108] Melissa R McGuirl, Samuel Pattillo Smith, Björn Sandstede, and Sohini Ramachandran. Detecting shared genetic architecture among multiple phenotypes by hierarchical clustering of gene-level association statistics. Genetics, 2020.

[109] Maria Maddalena Barbieri, James O Berger, et al. Optimal predictive model selection. The annals of statistics, 32(3):870-897, 2004.

[110] Derek Klarin, Scott M Damrauer, Kelly Cho, Yan V Sun, Tanya M Teslovich, Jacqueline Honerlaw, David R Gagnon, Scott L DuVall, Jin Li, Gina M Peloso, et al. Genetics of blood lipids among 300,000 multi-ethnic participants of the million veteran program. Nature genetics, 50(11):1514-1523, 2018.

[111] Paul S De Vries, Michael R Brown, Amy R Bentley, Yun J Sung, Thomas W Winkler, Ioanna Ntalla, Karen Schwander, Aldi T Kraja, Xiuqing Guo, Nora Franceschini, et al. Multiancestry genomewide association study of lipid levels incorporating gene-alcohol interactions. American journal of epidemiology, 188(6):1033-1054, 2019.

[112] Raymond Noordam, Maxime M Bos, Heming Wang, Thomas W Winkler, Amy R Bentley, Tuomas O Kilpeläinen, Paul S de Vries, Yun Ju Sung, Karen Schwander, Brian E Cade, et al. Multi-ancestry 
sleep-by-snp interaction analysis in 126,926 individuals reveals lipid loci stratified by sleep duration. Nature communications, 10(1):1-13, 2019.

[113] Tom G Richardson, Eleanor Sanderson, Tom M Palmer, Mika Ala-Korpela, Brian A Ference, George Davey Smith, and Michael V Holmes. Evaluating the relationship between circulating lipoprotein lipids and apolipoproteins with risk of coronary heart disease: A multivariable mendelian randomisation analysis. PLoS medicine, 17(3):e1003062, 2020.

[114] Thomas J Hoffmann, Elizabeth Theusch, Tanushree Haldar, Dilrini K Ranatunga, Eric Jorgenson, Marisa W Medina, Mark N Kvale, Pui-Yan Kwok, Catherine Schaefer, Ronald M Krauss, et al. A large electronic-health-record-based genome-wide study of serum lipids. Nature genetics, 50(3):401$413,2018$.

[115] Alexander M Kulminski, Jian Huang, Yury Loika, Konstantin G Arbeev, Olivia Bagley, Arseniy Yashkin, Matt Duan, and Irina Culminskaya. Strong impact of natural-selection-free heterogeneity in genetics of age-related phenotypes. Aging (Albany NY), 10(3):492, 2018.

[116] Aldi T Kraja, Dhananjay Vaidya, James S Pankow, Mark O Goodarzi, Themistocles L Assimes, Iftikhar J Kullo, Ulla Sovio, Rasika A Mathias, Yan V Sun, Nora Franceschini, et al. A bivariate genome-wide approach to metabolic syndrome: Stampeed consortium. Diabetes, 60(4):1329-1339, 2011.

[117] Cassandra N Spracklen, Peng Chen, Young Jin Kim, Xu Wang, Hui Cai, Shengxu Li, Jirong Long, Ying Wu, Ya Xing Wang, Fumihiko Takeuchi, et al. Association analyses of east asian individuals and trans-ancestry analyses with european individuals reveal new loci associated with cholesterol and triglyceride levels. Human molecular genetics, 26(9):1770-1784, 2017.

[118] Makoto Kurano, Kazuhisa Tsukamoto, Shigeo Kamitsuji, Naoyuki Kamatani, Masumi Hara, Toshio Ishikawa, Bong-Jo Kim, Sanghoon Moon, Young Jin Kim, and Tamio Teramoto. Genome-wide association study of serum lipids confirms previously reported associations as well as new associations of common snps within pcsk7 gene with triglyceride. Journal of human genetics, 61(5):427-433, 2016.

[119] Amy R Bentley, Yun J Sung, Michael R Brown, Thomas W Winkler, Aldi T Kraja, Ioanna Ntalla, Karen Schwander, Daniel I Chasman, Elise Lim, Xuan Deng, et al. Multi-ancestry genome-wide gene-smoking interaction study of 387,272 individuals identifies new loci associated with serum lipids. Nature genetics, 51(4):636-648, 2019. 
[120] Ida Surakka, Momoko Horikoshi, Reedik Mägi, Antti-Pekka Sarin, Anubha Mahajan, Vasiliki Lagou, Letizia Marullo, Teresa Ferreira, Benjamin Miraglio, Sanna Timonen, et al. The impact of lowfrequency and rare variants on lipid levels. Nature genetics, 47(6):589-597, 2015.

[121] Tanya M Teslovich, Kiran Musunuru, Albert V Smith, Andrew C Edmondson, Ioannis M Stylianou, Masahiro Koseki, James P Pirruccello, Samuli Ripatti, Daniel I Chasman, Cristen J Willer, et al. Biological, clinical and population relevance of 95 loci for blood lipids. Nature, 466(7307):707-713, 2010.

[122] Jake R Conway, Alexander Lex, and Nils Gehlenborg. Upsetr: an r package for the visualization of intersecting sets and their properties. Bioinformatics, 33(18):2938-2940, 2017.

[123] Terhi Kettunen, Carita Eklund, Mika Kähönen, Antti Jula, Hannu Päivä, Leo-Pekka Lyytikäinen, Mikko Hurme, and Terho Lehtimäki. Polymorphism in the c-reactive protein (crp) gene affects crp levels in plasma and one early marker of atherosclerosis in men: The health 2000 survey. Scandinavian journal of clinical and laboratory investigation, 71(5):353-361, 2011.

[124] Amy Z Fan, Ajay Yesupriya, Man-huei Chang, Meaghan House, Jing Fang, Renée Ned, Donald Hayes, Nicole F Dowling, and Ali H Mokdad. Gene polymorphisms in association with emerging cardiovascular risk markers in adult women. BMC medical genetics, 11(1):6, 2010.

[125] E Komurcu-Bayrak, N Erginel-Unaltuna, A Onat, B Ozsait, C Eklund, M Hurme, N Mononen, R Laaksonen, G Hergenc, and T Lehtimäki. Association of c-reactive protein (crp) gene allelic variants with serum crp levels and hypertension in turkish adults. Atherosclerosis, 206(2):474-479, 2009.

[126] S-N Chang, C-T Tsai, C-K Wu, J-K Lee, L-P Lai, S-W Huang, L-Y Huang, C-D Tseng, J-L Lin, F-T Chiang, et al. A functional variant in the promoter region regulates the c-reactive protein gene and is a potential candidate for increased risk of atrial fibrillation. Journal of internal medicine, 272(3): 305-315, 2012.

[127] Hyoun-Ah Kim, Hye-Young Chun, Seung-Hyun Kim, Hae-Sim Park, and Chang-Hee Suh. C-reactive protein gene polymorphisms in disease susceptibility and clinical manifestations of korean systemic lupus erythematosus. The Journal of rheumatology, 36(10):2238-2243, 2009.

[128] Abbas Dehghan, Josée Dupuis, Maja Barbalic, Joshua C Bis, Gudny Eiriksdottir, Chen Lu, Niina Pellikka, Henri Wallaschofski, Johannes Kettunen, Peter Henneman, et al. Meta-analysis of genome-wide association studies in 80000 subjects identifies multiple loci for c-reactive protein levels. Circulation, 123(7):731-738, 2011. 
[129] Paul M Ridker, Guillaume Pare, Alex Parker, Robert YL Zee, Jacqueline S Danik, Julie E Buring, David Kwiatkowski, Nancy R Cook, Joseph P Miletich, and Daniel I Chasman. Loci related to metabolic-syndrome pathways including lepr, hnf1a, il6r, and gckr associate with plasma c-reactive protein: the women's genome health study. The American Journal of Human Genetics, 82(5):1185$1192,2008$.

[130] Symen Ligthart, Ahmad Vaez, Urmo Võsa, Maria G Stathopoulou, Paul S De Vries, Bram P Prins, Peter J Van der Most, Toshiko Tanaka, Elnaz Naderi, Lynda M Rose, et al. Genome analyses ofi 200,000 individuals identify 58 loci for chronic inflammation and highlight pathways that link inflammation and complex disorders. The American Journal of Human Genetics, 103(5):691-706, 2018.

[131] Alex P Reiner, Sandra Beleza, Nora Franceschini, Paul L Auer, Jennifer G Robinson, Charles Kooperberg, Ulrike Peters, and Hua Tang. Genome-wide association and population genetic analysis of c-reactive protein in african american and hispanic american women. The American Journal of Human Genetics, 91(3):502-512, 2012.

[132] Paul Elliott, John C Chambers, Weihua Zhang, Robert Clarke, Jemma C Hopewell, John F Peden, Jeanette Erdmann, Peter Braund, James C Engert, Derrick Bennett, et al. Genetic loci associated with c-reactive protein levels and risk of coronary heart disease. Jama, 302(1):37-48, 2009.

[133] Deog Kyeom Kim, Michael H Cho, Craig P Hersh, David A Lomas, Bruce E Miller, Xiangyang Kong, Per Bakke, Amund Gulsvik, Alvar Agustí, Emiel Wouters, et al. Genome-wide association analysis of blood biomarkers in chronic obstructive pulmonary disease. American journal of respiratory and critical care medicine, 186(12):1238-1247, 2012.

[134] William J Astle, Heather Elding, Tao Jiang, Dave Allen, Dace Ruklisa, Alice L Mann, Daniel Mead, Heleen Bouman, Fernando Riveros-Mckay, Myrto A Kostadima, et al. The allelic landscape of human blood cell trait variation and links to common complex disease. Cell, 167(5):1415-1429, 2016.

[135] Juraj Sokol, Kamil Biringer, Maria Skerenova, Miroslav Hasko, Lenka Bartosova, Jan Stasko, Jan Danko, and Peter Kubisz. Platelet aggregation abnormalities in patients with fetal losses: the gp6 gene polymorphism. Fertility and sterility, 98(5):1170-1174, 2012.

[136] Andrew D Johnson, Lisa R Yanek, Ming-Huei Chen, Nauder Faraday, Martin G Larson, Geoffrey Tofler, Shiow J Lin, Aldi T Kraja, Michael A Province, Qiong Yang, et al. Genome-wide meta-analyses identifies seven loci associated with platelet aggregation in response to agonists. Nature genetics, 42 (7):608-613, 2010 . 
[137] Takao Shimizu. Lipid mediators in health and disease: enzymes and receptors as therapeutic targets for the regulation of immunity and inflammation. Annual review of pharmacology and toxicology, 49: 123-150, 2009.

[138] Yuji Shimizu, Kazuhiko Arima, Yuko Noguchi, Shin-Ya Kawashiri, Hirotomo Yamanashi, Mami Tamai, Yasuhiro Nagata, and Takahiro Maeda. Potential mechanisms underlying the association between single nucleotide polymorphism (brap and aldh2) and hypertension among elderly japanese population. Scientific Reports, 10(1):1-9, 2020.

[139] John D Eicher, Nathalie Chami, Tim Kacprowski, Akihiro Nomura, Ming-Huei Chen, Lisa R Yanek, Salman M Tajuddin, Ursula M Schick, Andrew J Slater, Nathan Pankratz, et al. Platelet-related variants identified by exomechip meta-analysis in 157,293 individuals. The American Journal of Human Genetics, 99(1):40-55, 2016.

[140] Rehan Qayyum, Beverly M Snively, Elad Ziv, Michael A Nalls, Yongmei Liu, Weihong Tang, Lisa R Yanek, Leslie Lange, Michele K Evans, Santhi Ganesh, et al. A meta-analysis and genome-wide association study of platelet count and mean platelet volume in african americans. PLoS Genet, 8(3): e1002491, 2012.

[141] Cristen J Willer, Ellen M Schmidt, Sebanti Sengupta, Gina M Peloso, Stefan Gustafsson, Stavroula Kanoni, Andrea Ganna, Jin Chen, Martin L Buchkovich, Samia Mora, et al. Discovery and refinement of loci associated with lipid levels. Nature genetics, 45(11):1274, 2013.

[142] Rita PS Middelberg, Manuel AR Ferreira, Anjali K Henders, Andrew C Heath, Pamela AF Madden, Grant W Montgomery, Nicholas G Martin, and John B Whitfield. Genetic variants in lpl, oasl and tomm40/apoe-c1-c2-c4 genes are associated with multiple cardiovascular-related traits. BMC medical genetics, 12(1):123, 2011.

[143] Sekar Kathiresan, Olle Melander, Candace Guiducci, Aarti Surti, Noël P Burtt, Mark J Rieder, Gregory M Cooper, Charlotta Roos, Benjamin F Voight, Aki S Havulinna, et al. Six new loci associated with blood low-density lipoprotein cholesterol, high-density lipoprotein cholesterol or triglycerides in humans. Nature genetics, 40(2):189-197, 2008.

[144] Jaspal S Kooner, John C Chambers, Carlos A Aguilar-Salinas, David A Hinds, Craig L Hyde, Gregory R Warnes, Francisco J Gómez Pérez, Kelly A Frazer, Paul Elliott, James Scott, et al. Genomewide scan identifies variation in mlxipl associated with plasma triglycerides. Nature genetics, 40(2): $149,2008$. 
[145] Sekar Kathiresan, Cristen J Willer, Gina M Peloso, Serkalem Demissie, Kiran Musunuru, Eric E Schadt, Lee Kaplan, Derrick Bennett, Yun Li, Toshiko Tanaka, et al. Common variants at 30 loci contribute to polygenic dyslipidemia. Nature genetics, 41(1):56-65, 2009.

[146] Maria Keller, Dorit Schleinitz, Julia Förster, Anke Tönjes, Yvonne Böttcher, Antje Fischer-Rosinsky, Jana Breitfeld, Kerstin Weidle, Nigel W Rayner, Ralph Burkhardt, et al. Thoc5: a novel gene involved in hdl-cholesterol metabolism. Journal of lipid research, 54(11):3170-3176, 2013.

[147] Richa Saxena, Benjamin F Voight, Valeriya Lyssenko, Noël P Burtt, Paul IW de Bakker, Hong Chen, Jeffrey J Roix, Sekar Kathiresan, Joel N Hirschhorn, Mark J Daly, et al. Genome-wide association analysis identifies loci for type 2 diabetes and triglyceride levels. Science, 316(5829):1331-1336, 2007.

[148] Yurii S Aulchenko, Samuli Ripatti, Ida Lindqvist, Dorret Boomsma, Iris M Heid, Peter P Pramstaller, Brenda WJH Penninx, A Cecile JW Janssens, James F Wilson, Tim Spector, et al. Loci influencing lipid levels and coronary heart disease risk in 16 european population cohorts. Nature genetics, $41(1)$ : 47, 2009.

[149] Rubina Tabassum, Joel T Rämö, Pietari Ripatti, Jukka T Koskela, Mitja Kurki, Juha Karjalainen, Priit Palta, Shabbeer Hassan, Javier Nunez-Fontarnau, Tuomo TJ Kiiskinen, et al. Genetic architecture of human plasma lipidome and its link to cardiovascular disease. Nature communications, 10(1):1-14, 2019.

[150] Liang He, Yelena Kernogitski, Irina Kulminskaya, Yury Loika, Konstantin G Arbeev, Elena Loiko, Olivia Bagley, Matt Duan, Arseniy Yashkin, Svetlana V Ukraintseva, et al. Pleiotropic meta-analyses of longitudinal studies discover novel genetic variants associated with age-related diseases. Frontiers in genetics, 7:179, 2016.

[151] Dawn M Waterworth, Sally L Ricketts, Kijoung Song, Li Chen, Jing Hua Zhao, Samuli Ripatti, Yurii S Aulchenko, Weihua Zhang, Xin Yuan, Noha Lim, et al. Genetic variants influencing circulating lipid levels and risk of coronary artery disease. Arteriosclerosis, thrombosis, and vascular biology, 30(11): 2264-2276, 2010.

[152] Gina M Peloso, Paul L Auer, Joshua C Bis, Arend Voorman, Alanna C Morrison, Nathan O Stitziel, Jennifer A Brody, Sumeet A Khetarpal, Jacy R Crosby, Myriam Fornage, et al. Association of lowfrequency and rare coding-sequence variants with blood lipids and coronary heart disease in 56,000 whites and blacks. The American Journal of Human Genetics, 94(2):223-232, 2014. 
[153] Tuomas O Kilpeläinen, Amy R Bentley, Raymond Noordam, Yun Ju Sung, Karen Schwander, Thomas W Winkler, Hermina Jakupović, Daniel I Chasman, Alisa Manning, Ioanna Ntalla, et al. Multi-ancestry study of blood lipid levels identifies four loci interacting with physical activity. Nature communications, 10(1):1-11, 2019.

[154] Yoichiro Kamatani, Koichi Matsuda, Yukinori Okada, Michiaki Kubo, Naoya Hosono, Yataro Daigo, Yusuke Nakamura, and Naoyuki Kamatani. Genome-wide association study of hematological and biochemical traits in a japanese population. Nature genetics, 42(3):210, 2010.

[155] Marc A Coram, Qing Duan, Thomas J Hoffmann, Timothy Thornton, Joshua W Knowles, Nicholas A Johnson, Heather M Ochs-Balcom, Timothy A Donlon, Lisa W Martin, Charles B Eaton, et al. Genome-wide characterization of shared and distinct genetic components that influence blood lipid levels in ethnically diverse human populations. The American Journal of Human Genetics, 92(6): 904-916, 2013.

[156] Deepti Gurdasani, Tommy Carstensen, Segun Fatumo, Guanjie Chen, Chris S Franklin, Javier PradoMartinez, Heleen Bouman, Federico Abascal, Marc Haber, Ioanna Tachmazidou, et al. Uganda genome resource enables insights into population history and genomic discovery in africa. Cell, 179(4):984-1002, 2019.

[157] Elisabeth M van Leeuwen, Aniko Sabo, Joshua C Bis, Jennifer E Huffman, Ani Manichaikul, Albert V Smith, Mary F Feitosa, Serkalem Demissie, Peter K Joshi, Qing Duan, et al. Meta-analysis of 49 549 individuals imputed with the 1000 genomes project reveals an exonic damaging variant in angptl4 determining fasting tg levels. Journal of medical genetics, 53(7):441-449, 2016.

[158] Robert Perneczky, Panagiotis Alexopoulos, and Alzheimer's Disease euroimaging Initiative. Cerebrospinal fluid bace1 activity and markers of amyloid precursor protein metabolism and axonal degeneration in alzheimer's disease. Alzheimer's \& Dementia, 10:S425-S429, 2014.

[159] Christoph Heiring, Björn Dahlbäck, and Yves A Muller. Ligand recognition and homophilic interactions in tyro3 structural insights into the axl/tyro3 receptor tyrosine kinase family. Journal of Biological Chemistry, 279(8):6952-6958, 2004. 